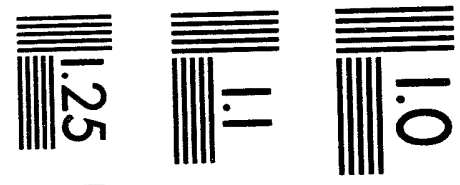

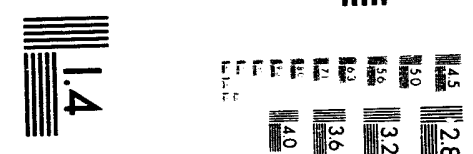

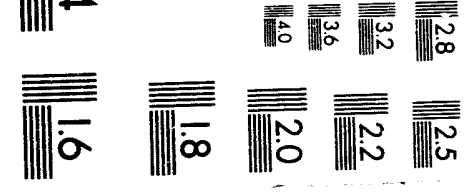



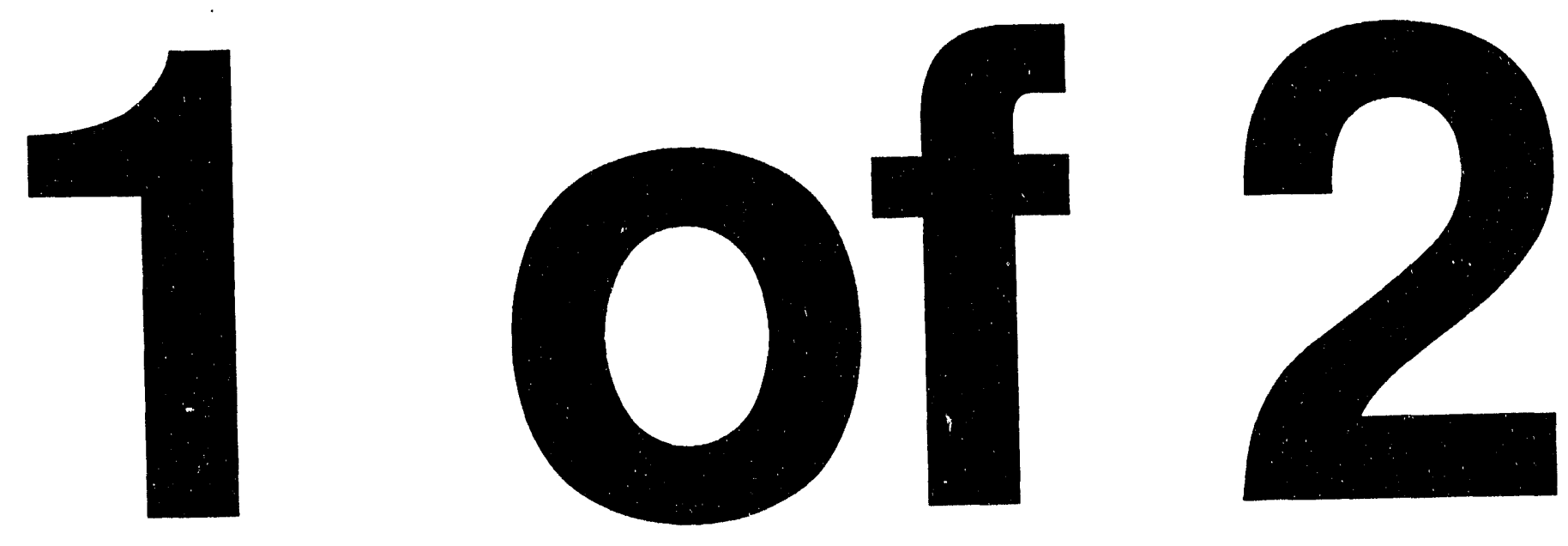


\title{
MATERIAL IDENTIFICATION TECHNOLOGY (MIT) Concept Technical Feasibility Study
}

September 1993

\author{
J. L. Jones \\ Y. D. Harker \\ W. Y. Yoon \\ L. O. Johnson
}




\section{DISCLAIMER}

This report was prepared as an account of work sponsored by an agency of the United States Government. Neither the United States Government nor any agency thereof, nor any of their employees, makes any warranty, express or implied, or assumes any legal liability or responsibility for the accuracy, completeness, or usefulness of any information, apparatus, product or process disclosed, or represents that its use would not intringe privately owned rights. References herein to any specific commercial product, process, or service by trade name, trademark, manufacturer, or otherwise, does not necessarily constitute or imply its endorsement, recommendation, or favoring by the United States Government or any agency thereof. The views and opinions of authors expressed herein do not necessarily state or reflect those of the United States Government or any agency thereot. 
TABLE OF CONTENTS

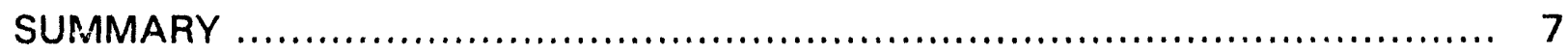

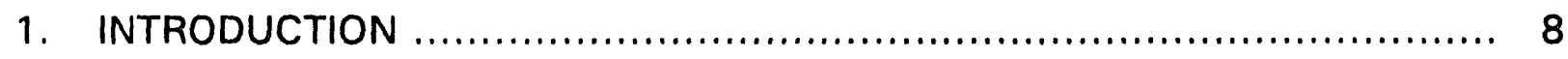

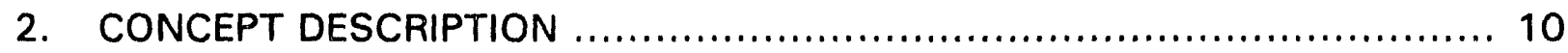

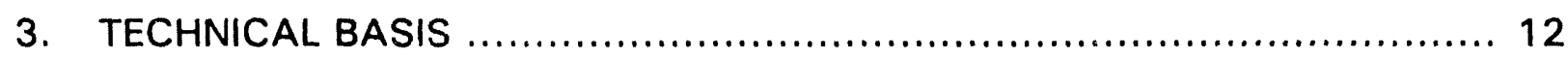

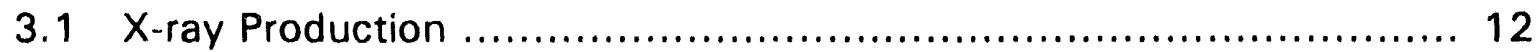

3.2 Neutron Production and Interactions ............................... 12

3.2.1 Photoneutron/photofission process .......................... 12

3.2.2 Fundamental Timing ........................................ 16

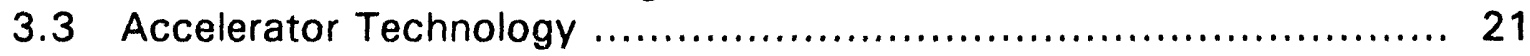

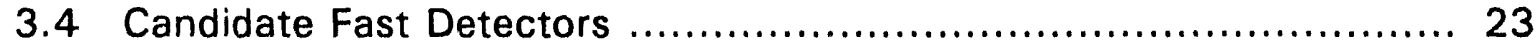

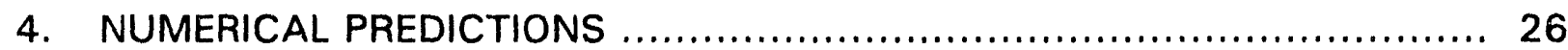

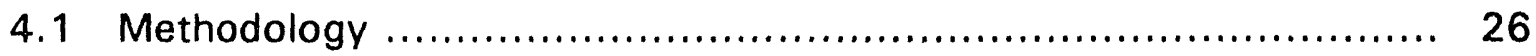

4.2 Model Description .................................................. 26

4.3 Interrogated Objects Description ................................. 29

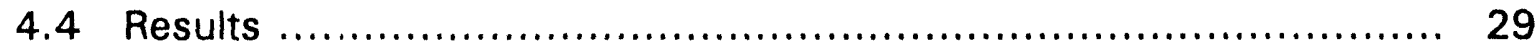

4.4.1 Neutron Production ...................................... 29

4.4.2 Neutron Response at the Detector ........................... 33

4.4.3 Gamma Response at the Detector ......................... 33

5. TEMPORAL DETECTOR RESPONSE ASSESSMENTS ..................... 56

5.1 Experiment Description ............................................... 56

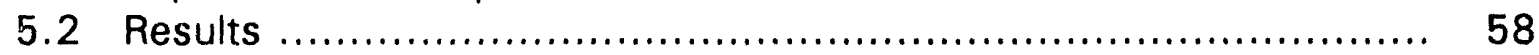

5.3 Discussion of Results ............................................... 61

6. POTENTIAL DETFCTION SYSTEM CONCEPT ............................ 68



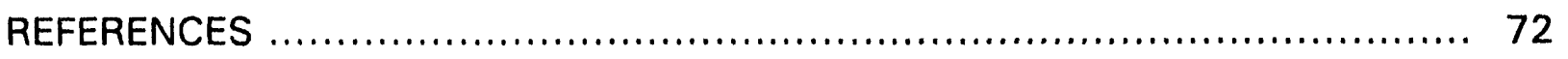

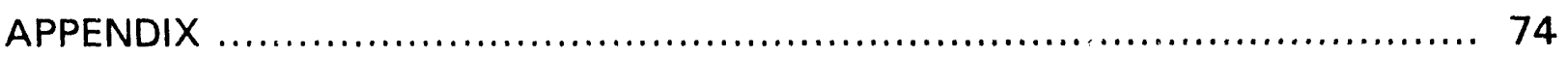




\section{FIGURES}

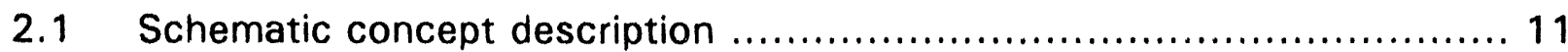

3.1 Calculated bremsstrahlung X-ray spectra for various electron beam energies

3.2 X-ray intensity distributions for various electron beam energies

3.3 The prompt neutron fission spectrum

3.4 General concept timing considerations for $X$-rays, gamma, and neutrons for an input electron beam impulse

3.5 Basic geometry for primary concept components 19

3.6 X-ray emission rates from high-Z convertors (targets) $\ldots \ldots \ldots \ldots \ldots \ldots \ldots . \ldots 22$

4.1 Numerical modeling methodology ..................................... 27



4.3 Total neutron production and average neutron energy versus electron beam energy for the $0.5-\mathrm{cm}$ thick beryllium shell $(R o=50.5 \mathrm{~cm})$ in the calculational model

4.4 Neutron production spectra of the $0.5-\mathrm{cm}$ thick beryllium shell

$(R o=50.5 \mathrm{~cm})$ for various electron beam energies

4.5 Detector location temporal neutron response for various object

materials and using 13-MeV electrons (i.e., Cases 1-3)

4.6 Detector location neutron spectral responses for 13-MeV electrons and mustard gas (Case 1)

4.7 Detector location temporal neutron responses for mustard gas with several electron beam energies and object sizes (i.e., Cases 1,4,5, and 8)

4.8 Detector location temporal gamma responses for various object materials and 13-MeV electrons (i.e., Cases 1-3)

4.9 Detector location temporal gamma responses for various object materials and 8-MeV electrons (i.e., Cases 1-3) 
4.10 Detector location gamma responses (0 - $15 \mathrm{~ns})$ for mustard gas object $(R=50-\mathrm{cm})$ and $13-\mathrm{MeV}$ electrons (Case 1)

4.11 Detector location gamma responses (80 - 5000 ns) for mustard gas object $(R=50-\mathrm{cm}$ ) and 13-MeV electrons (Case 1) 40

4.12 Detector location gamma responses (0 - $15 \mathrm{~ns}$ ) for concrete object $(R=50-\mathrm{cm})$ and $13-\mathrm{MeV}$ electrons (Case 2)

4.13 Detector location gamma responses ( 80 - $5000 \mathrm{~ns}$ ) for concrete object $(R=50-\mathrm{cm})$ and $13-\mathrm{MeV}$ electrons (Case 2 )

4.14 Detector location gamma responses (0 - $15 \mathrm{~ns}$ ) for Fe/Poly object $(R=45 / 50-\mathrm{cm})$ and $13-\mathrm{MeV}$ electrons (Case 3)

4.15 Detector location gamma responses (80 - 5000 ns) for Fe/Poly object $(R=45 / 50-\mathrm{cm}$ ) and $13-\mathrm{MeV}$ electrons (Case 3 )

4.16 Detector location gamma responses (0 - $15 \mathrm{~ns})$ for mustard gas object $(R=25-\mathrm{cm})$ and $13-\mathrm{MeV}$ electrons (Case 4)

4.17 Detector location gamma responses (80 - $5000 \mathrm{~ns}$ ) for mustard gas object $(R=25-\mathrm{cm})$ and $13-\mathrm{MeV}$ electrons (Case 4)

4.18 Detector location gamma responses (0 - $15 \mathrm{~ns})$ for mustard gas object $(R=50-\mathrm{cm})$ and $8-\mathrm{MeV}$ electrons (Case 1 )

4.19 Detector location gamma responses ( 80 - $5000 \mathrm{~ns}$ ) for mustard gas object $(R=50-\mathrm{cm})$ and $8-\mathrm{MeV}$ electrons (Case 1 )

4.20 Detector location gamma responses (0 - $15 \mathrm{~ns}$ ) for concrete object $(R=50-\mathrm{cm})$ and $8-\mathrm{MeV}$ electrons (Case 2)

4.21 Detector location gamma responses ( 80 - $5000 \mathrm{~ns}$ ) for concrete object $(R=50-\mathrm{cm}$ ) and $8-\mathrm{MeV}$ electrons (Case 2) 50

4.22 Detector location gamma responses (0 - $15 \mathrm{~ns}$ ) for Fe/Poly object $(R=45 / 50-\mathrm{cm})$ and $8-\mathrm{MeV}$ electrons (Case 3 )

4.23 Detector location gamma responses ( $80-5000 \mathrm{~ns}$ ) for Fe/Poly object $(R=45 / 50-\mathrm{cm})$ and $8-\mathrm{MeV}$ electrons (Case 3 )

4.24 Detector location gamma responses (0 - $15 \mathrm{~ns}$ ) for mustard gas object $(R=25-\mathrm{cm})$ and $8-\mathrm{MeV}$ electrons (Case 4 ) 53 
TABLE OF CONTENTS





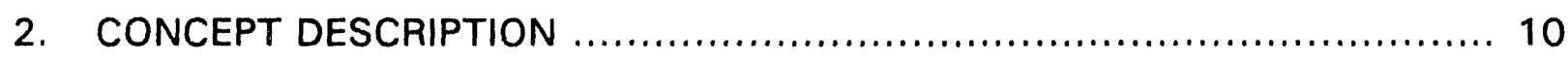

3. TECHNICAL BASIS ...................................................... 12

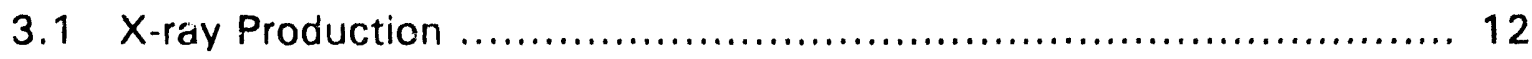

3.2 Neutron Production and Interactions …............................ 12

3.2.1 Photoneutron/photofission process ......................... 12

3.2.2 Fundamental Timing .......................................... 16



3.4 Candidate Fast Detectors ......................................... 23

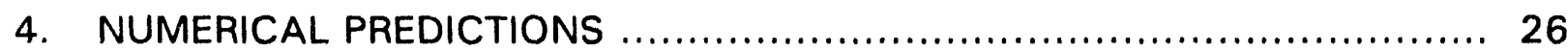

4.1 Methodology ......................................................... 26

4.2 Model Description ................................................. 26

4.3 Interrogated Objects Description .................................. 29

4.4 Results .............................................................. 29

4.4.1 Neutron Production ...................................... 29

4.4.2 Neutron Response at the Detector .......................... 33

4.4.3 Gamma Response at the Detector .......................... 33

5. TEMPORAL DETECTOR RESPONSE ASSESSMENTS $\ldots \ldots \ldots \ldots \ldots \ldots \ldots \ldots . \ldots \ldots$

5.1 Experiment Description .......................................... 56

5.2 Results ................................................................ 58

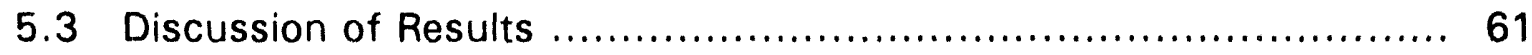

6. POTENTIAL DETECTION SYSTEM CONCEPT ................................ 68

7. RECOMMENDATIONS …................................................. 71

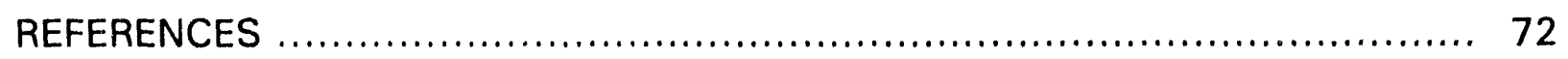

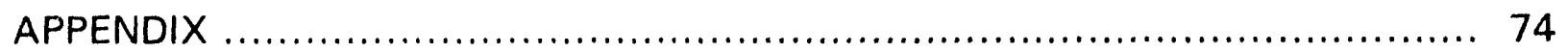




\section{FIGURES}

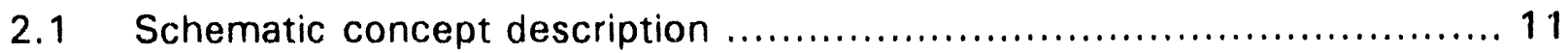

3.1 Calculated bremsstrahlung $X$-ray spectra for various electron beam energies ............................................................. 13

3.2 X-ray intensity distributions for various electron beam energies .......... 14

3.3 The prompt neutron fission spectrum ................................ 15

3.4 General concept timing considerations for $X$-rays, gamma, and neutrons for an input electron beam impulse ............................. 18

3.5 Basic geometry for primary concept components ....................... 19

3.6 X-ray emission rates from high-Z convertors (targets) $\ldots \ldots \ldots \ldots \ldots \ldots \ldots . \ldots 22$

$4.1 \quad$ Numerical modeling methodology ...................................... 27

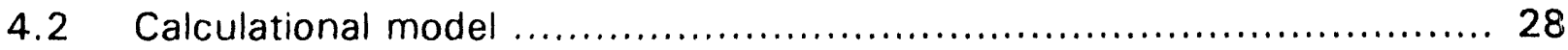

4.3 Total neutron production and average neutron energy versus electron beam energy for the $0.5-\mathrm{cm}$ thick beryllium shell $(R o=50.5 \mathrm{~cm}$ ) in the calculational model

4.4 Neutron production spectra of the $0.5-\mathrm{cm}$ thick beryllium shell $\left(R_{0}=50.5 \mathrm{~cm}\right)$ for various electron beam energies

4.5 Detector location temporal neutron response for various object materials and using 13-MeV electrons (i.e., Cases 1-3)

4.6 Detector location neutron spectral responses for $13-\mathrm{MeV}$ electrons and mustard gas (Case 1)

4.7 Detector location temporal neutron responses for mustard gas with severai electron beam energies and object sizes (i.e., Cases 1,4,5, and 8)

4.8 Detector location temporal gamma responses for various object materials and 13-MeV electrons (i.e., Cases 1-3)

4.9 Detector location temporal gamma responses for various object materials and 8-MeV electrons (i.e., Cases 1-3) 
4.10 Detector location gamma responses (0 - $15 \mathrm{~ns})$ for mustard gas object $(R=50-\mathrm{cm})$ and 13-MeV electrons (Case 1 )

4.11 Detector location gamma responses ( 80 - 5000 ns) for mustard gas object $(R=50-\mathrm{cm})$ and $13-\mathrm{MeV}$ electrons (Case 1 ) ...

4.12 Detector location gamma responses (0 - $15 \mathrm{~ns})$ for concrete object $(R=50-\mathrm{cm})$ and $13-\mathrm{MeV}$ electrons (Case 2)

4.13 Detector location gamma responses ( 80 - $5000 \mathrm{~ns}$ ) for concrete object $(R=50-\mathrm{cm})$ and $13-\mathrm{MeV}$ electrons (Case 2)

4.14 Detector location gamma responses (0 - $15 \mathrm{~ns}$ ) for Fe/Poly object $(R=45 / 50-\mathrm{cm})$ and $13-\mathrm{MeV}$ electrons (Case 3 )

4.15 Detector location gamma responses ( $80-5000 \mathrm{~ns}$ ) for Fe/Poly object $(R=45 / 50-\mathrm{cm})$ and $13-\mathrm{MeV}$ electrons (Case 3)

4.16 Detector location gamma responses (0 - $15 \mathrm{~ns})$ for mustard gas object $(R=25-\mathrm{cm})$ and $13-\mathrm{MeV}$ electrons (Case 4 )

4.17 Detector location gamma responses (80-5000 ns) for mustard gas object $(R=25-\mathrm{cm})$ and 13-MeV electrons (Case 4) 46

4.18 Detector location gamma responses (0 - $15 \mathrm{~ns}$ ) for mustard gas object $(R=50-\mathrm{cm})$ and $8-\mathrm{MeV}$ electrons (Case 1)

4.19 Detector location gamma responses ( $80-5000 \mathrm{~ns}$ ) for mustard gas object $(R=50-\mathrm{cm})$ and $8-\mathrm{MeV}$ electrons (Case 1 ) 48

4.20 Detector location gamma responses (0 - $15 \mathrm{~ns})$ for concrete object $(R=50-\mathrm{cm})$ and $8-\mathrm{MeV}$ electrons (Case 2) 49

4.21 Detector location gamma responses (80-5000 ns) for concrete object $(R=50-\mathrm{cm})$ and $8-\mathrm{MeV}$ electrons (Case 2)

4.22 Detector location gamma responses (0 - $15 \mathrm{~ns}$ ) for Fe/Poly object $(R=45 / 50-\mathrm{cm}$ ) and $8-\mathrm{MeV}$ electrons (Case 3)

4.23 Detector location gamma responses (80 - $5000 \mathrm{~ns}$ ) for Fe/Poly object $(R=45 / 50-\mathrm{cm})$ and $8-\mathrm{MeV}$ electrons (Case 3 )

4.24 Detector location gamma responses (0 - $15 \mathrm{~ns}$ ) for mustard gas object $(R=25-\mathrm{cm})$ and $8-\mathrm{MeV}$ electrons (Case 4) 53 
4.25 Detector location gamma responses ( $80-5000 \mathrm{~ns}$ ) for mustard gas object $(R=25-\mathrm{cm})$ and $8-\mathrm{MeV}$ electrons (Case 4)

5.1 "Plan-view" of experimental configuration ............................. 57

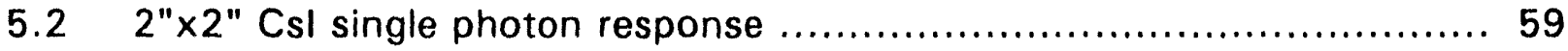

$5.3 \quad 2 " \times 2 "$ BC-418 single photon response ....................................60 60

$5.42 " \times 2 "$ Csl X-ray flash response for 12 ampere operation ................ 62

5.52 2"x2" BC-418 X-ray flash response for 12 ampere operation .............63 63

$5.62 " \times 2 "$ BHTP X-ray flash response for 12 ampere operation ............... 64

5.7 BC-418 and BHTP relative $X$-ray flash response comparisons. (BHTP has faster recovery time.) ..................................................6 65

6.1 Fast detection and acquisition system ............................... 69 


\section{TABLES}

3.1 Representative photoneutron/photofission production nuclides .......... 17

3.2 Selected neutron energy and corresponding speed $\ldots \ldots \ldots \ldots \ldots \ldots \ldots \ldots . \ldots 20$

3.3 Detector fast component energy resolution .......................... 25

4.1 Material composition of interrogated objects modeled .................. 30

4.2 Selected results for various cases assessed $\ldots \ldots \ldots \ldots \ldots \ldots \ldots \ldots \ldots \ldots \ldots \ldots$

4.3 Correspondence between included figures and cases assessed .......... 55

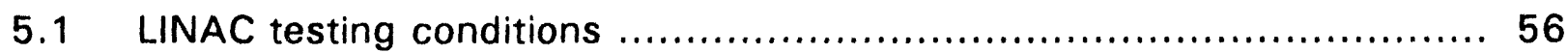

5.2 Scintillator time response parameters for single photon interactions ..... 58

5.3 Scintillator time response parameters for electron LINAC X-ray flash .... 66 


\section{SUMMARY}

A novel electron accelerator-based, active interrogation concept capable of nondestructive elemental analysis is shown to be feasible. The concept, consisting of a pulsed, electron accelerator and a gamma detection system, is fully described in this report. The descriptions include inherent concept advantages, $\mathrm{X}$-ray and neutron production mechanisms, neutron interaction processes resulting in characteristic, secondary gamma emissions, basic timing considerations, and requirements for accelerator and detection technology.

Numerical predictions of the neutron and photon detector responses for various interrogation object sizes and materials, including mustard gas, concrete (structural material), and an iron/polyethylene composition (structural materials) are described in detail. For a representative interrogation application of a mustard gas-containing object, as many as 71 characteristic gamma events are predicted to occur at the detector per an accelerator pulse containing $10^{12}$ electrons. The initial inelastic scattering events, occurring immediately $(<20 \mathrm{~ns})$ after an accelerator pulse, are shown to produce approximately two orders of magnitude more response than the longer time response (dominated by neutron capture interactions).

For detecting neutron inelastic scattering events, the key system component, still to be developed, is a detection system capable of ultra-fast performance. Detection of the slower responding neutron capture gammas can utilize more conventional detector materials. Four candidate fast scintillation detectors: $\mathrm{BaF}_{2}$, pure (non-doped) CsI, BC-418, and a specialty plastic, BHTP were tested to characterize their temporal behavior following accelerator-produced bursts of highintensity, pulsed X-rays. Multiple gamma events in these detectors were experimentally observed using a fast oscilloscope with rates up to $40 \mathrm{MHz}$ (between sequential accelerator pulses). Results show that BHTP has the fastest response of all the detectors, but $\mathrm{BC}-418$ provides better energy resolution and is commercially available. For the inorganic fast scintillators tested (those having a strong photoelectric gamma response), $\mathrm{BaF}_{2}$ is too slow for this application and the pure Csl is the best if a baseline restorer can effectively suppress the tail of the $X$ ray flash or if the electron beam current can be sufficiently reduced to minimize the $X$-ray flash effect.

Finally, a proposed detection system applicable to this technology is presented along with recommendations regarding future work. 


\section{INTRODUCTION}

The Idaho National Engineering Laboratory (INEL) has initiated the design and development of a novel pulsed accelerator-based, active interrogation concept. The proposed concept, referred to as the Material Identification Technology (MIT), enables rapid (between accelerator pulses), non-destructive, elemental composition analysis of both nuclear and non-nuclear materials. MIT has been funded in FY-92 and -93 at INEL through the Advanced Concepts Program of the U.S. Department of Energy (DOE) Office of Research and Development. Applications of this technique include material monitoring in support of counter-proliferation activities, such as export controls (at domestic and international inspection locations), SNM controls, nuclear weapon dismantlement, and chemical weapon verification.

Material Identification Technology combines a pulsed, X-ray source (an electron accelerator) and a gamma detection system. The accelerator must maximize neutron production (pulse width, beam current, beam energy, and repetition rate) and minimize photon dose to the object. Current available accelerator technology can meet these requirements. The detection system must include detectors which provide adequate gamma energy resolution capability, rapid recovery after the initial X-ray interrogation pulse, and multiple single gamma event detection between accelerator pulses. Further research is required to develop the detection system.

Neutron activation and corresponding gamma spectroscopy has been a standard method for elemental identification for several decades. Typically, activation has been accomplished with a nuclear reactor, but other neutron sources can be used. Material identification has been performed with radioactive neutron sources ${ }^{19}$ and neutron generators (sealed tube deuterium/tritium, deuterium/deuterium devices ${ }^{20.21,22}$ ). Most non-reactor based technologies are interrogation limited by their neutron production capability. The proposed MIT concept is a pulsed neutron source capable of obtaining very high pulsed production rates since it utilizes easily accelerated electrons. Electron accelerator technology, available for this concept, has been utilized to produce intense neutron sources; for example, linear electron accelerator facilities have reached production rates of up to approximately $10^{19}$ neutrons/pulse ${ }^{23}$.

The advantages of the MIT technique over other technologies include:

(1) intense source of neutrons to provide fast interrogation times (especially as compared to inspections using radioactive sources),

(2) distributed neutron source to enable better utilization of interrogating neutrons,

(3) gamma spectra to provide unique elemental/material identification, 
(4) expansion of the number of nuclides which can be identified via the detection of induced neutron inelastic gammas in addition to the neutron capture gammas,

(5) direct compatibility with existing $X$-ray detection applications and inspection techniques,

(6) virtually no residual radiation when the system is not in use (i.e., no radioactive sources required),

(7) high signal-to-noise potential,

(8) gamma coincidence detection not required (will utilize accelerator pulses to trigger gamma detection system),

(9) high technology transfer potential,

(10) utilization of mostly standard, commercially-available technologies.

This report provides the initial feasibility assessment of the MIT concept. Section 2 describes the basic concept, while Section 3 presents a review of the basic technical issues and describes the current accelerator technology and candidate fast detectors. A description of the numerical methodology used in this assessment is included in Section 4 along with detailed performance predictions of various system configurations. Section 5 presents experimental results showing the temporal response of the candidate detectors to intense, pulsed $x$-rays.

Section 6 describes a proposed detection and acquisition system applicable to the MIT concept. Finally, recommendations regarding future efforts are included in Section 7. 


\section{CONCEPT DESCRIPTION}

The MIT technique (schematically presented in Figure 2.1) utilizes a pulsed, electron accelerator to produce energetic $X$-rays (i.e., bremsstrahlung). The pulsed $X$-rays interact in the interrogated object or a surrounding beryllium shell to generate photoneutrons directly within the target (and multiple photofission neutrons if nuclear materials are present). Beryllium has been used to date due to its extremely low photoneutron threshold energy (1.7 MeV). By using a sufficiently high electron beam energy (i.e., greater than $8-\mathrm{MeV}$ ), the resulting $X$ rays will stimulate neutron emissions in most materials. The resulting induced neutrons will interact in the interrogated object via neutron capture and inelastic reactions to generate characteristic secondary gamma emissions. Since the neutrons travel slower than the parent pulsed $X$-rays, there is a difference between the arrival times of the $X$-rays and the neutron at the detector. This allows differentiation between the neutron-induced, inelastic scattered, secondary gamma emissions resulting from the initial $X$-rays and those resulting from $X$-rays reflected from the object; hence, enabling detection of elements having very fast gamma emission characteristics, which could otherwise not be observed. Detection of the neutron-induced capture gamma emissions will not require this fast time response differentiation because of the longer time characteristics of these emissions. Detection requirements for each accelerator pulse range from ultra-fast responses (less than $50 \mathrm{~ns}$ total pulse response) for the detection of multiple inelastic scattering events to more conventional response times (> $500 \mathrm{~ns}$ ) for multiple capture events.

The detection and data acquisition system must be capable of detecting the characteristic gamma emissions between accelerator pulses and, for optimum operation, be able to detect and process multiple gamma pulses between accelerator pulses. The latter permits minimizing the $\mathrm{X}$-ray dose on the interrogated object and reducing interrogation times. The energy resolution of the detection system must be sufficient (less than 10 percent) to enable identification of detected gamma ray energies. Typically, fast gamma detection requirements suggest the use of fast scintillation detectors. The resulting gamma spectra will be analyzed using mostly standard spectroscopy methods to determine elemental composition. 


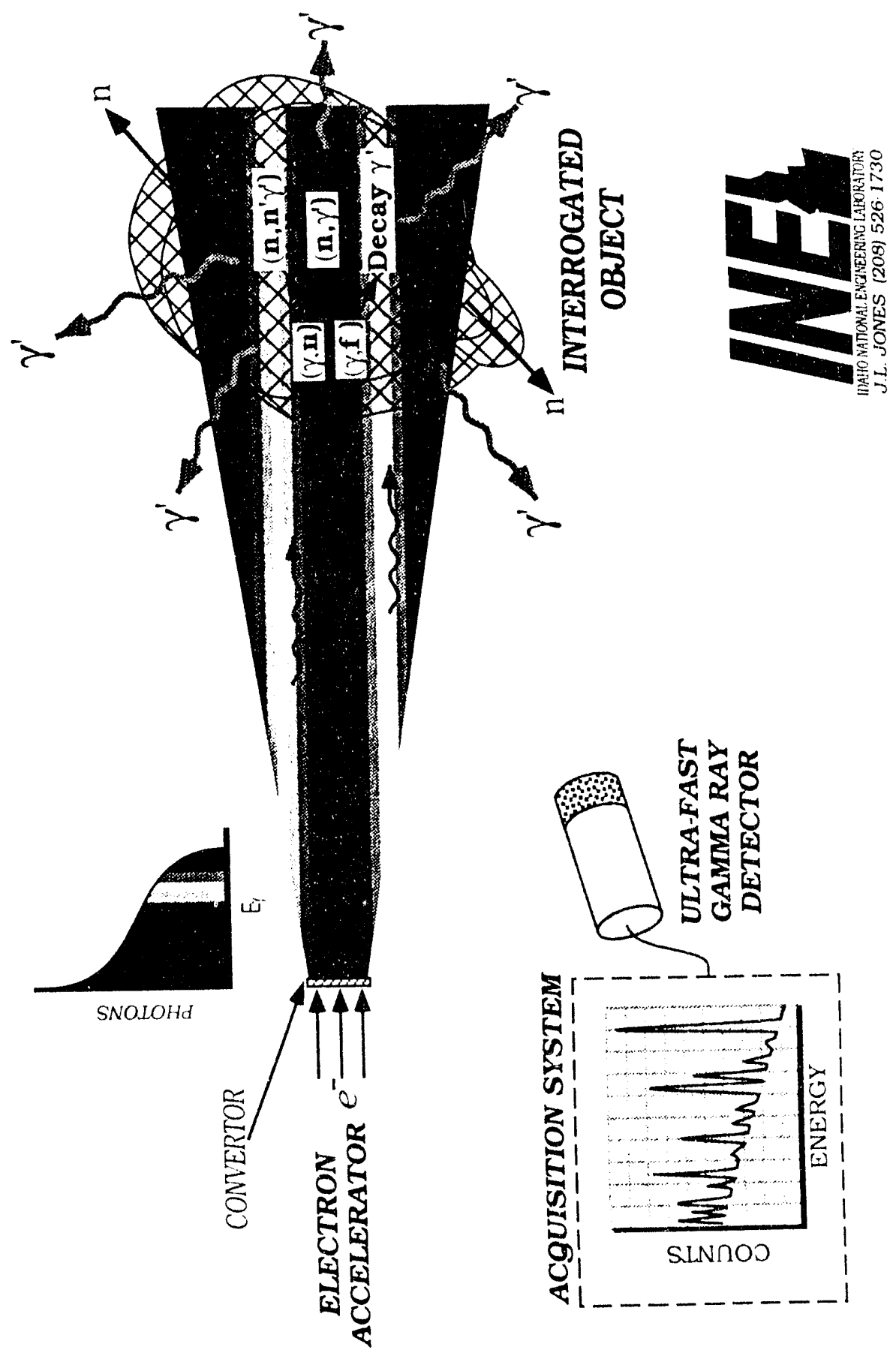

Figure 2.1 Schematic concept description. 


\section{TECHNICAL BASIS}

The interrogation concept feasibility study focuses on X-ray and neutron production mechanisms, accelerator technology, and applicable detectors.

\subsection{X-ray Production}

The energetic $X$-rays result from electron deceleration interactions within a high- $Z$ (atomic number) material, referred to as a convertor or target. As shown in Figure 3.1 for a composite convertor (gold and tungsten), the $X$-ray production process produces continuous photon (i.e., $X$-ray) energy spectra having a maximum energy corresponding to the maximum electron beam energy. In this report numerical predictions have utilized an unoptimized, but representative, $0.1 \mathrm{~cm}$ thick tungsten convertor. The $\mathrm{X}$-rays are primarily forward directed, and the radiation intensity becomes more forward peaked as the energy of the accelerator is increased (See Figure 3.2). High-Z collimator shields are used to help define/limit the forward directed cone angle. For the MIT application, this cone angle intensity response will efficiently utilize the source neutrons as a distributed source around and within the interrogated object.

\subsection{Neutron Production and Interactions}

The $X$-rays interact in the interrogated object and/or a nearby beryllium convertor to produce energetic neutrons via the photoneutron and/or photofission processes. In the photoneutron process, the energy difference between the parent X-ray energy and the photoneutron reaction energy threshold is available to the neutron as kinetic energy. In the photofission process, the induced fissions generate neutrons having a prompt neutron fission spectrum as indicated in Figure 3.3. Neutron production is essentially instantaneous, and the emitted neutrons are generated with a continuous energy distribution. Induced neutrons will interact with the interrogated object in proportion to the interaction probability of the materials in the object, resulting in the emissions of elemental characteristic gammas.

\subsubsection{Photoneutron/photofission Processes}

The interaction of energetic photons with matter has already been well characterized ${ }^{3}$. While several photon interactions can occur, the photoneutron and photofission interactions provide the source neutrons for this interrogation concept. The reaction cross-sections for these processes have been documented ${ }^{4}$ and have been utilized in another INEL arms control project ${ }^{5,6}$. These processes are endothermic, and most nuclei and their isotopes have differing threshold energies for the onset of a given process. Most non-nuclear materials will produce neutrons with exposure to energetic photons above 8-MeV. Photofission interactions occur 


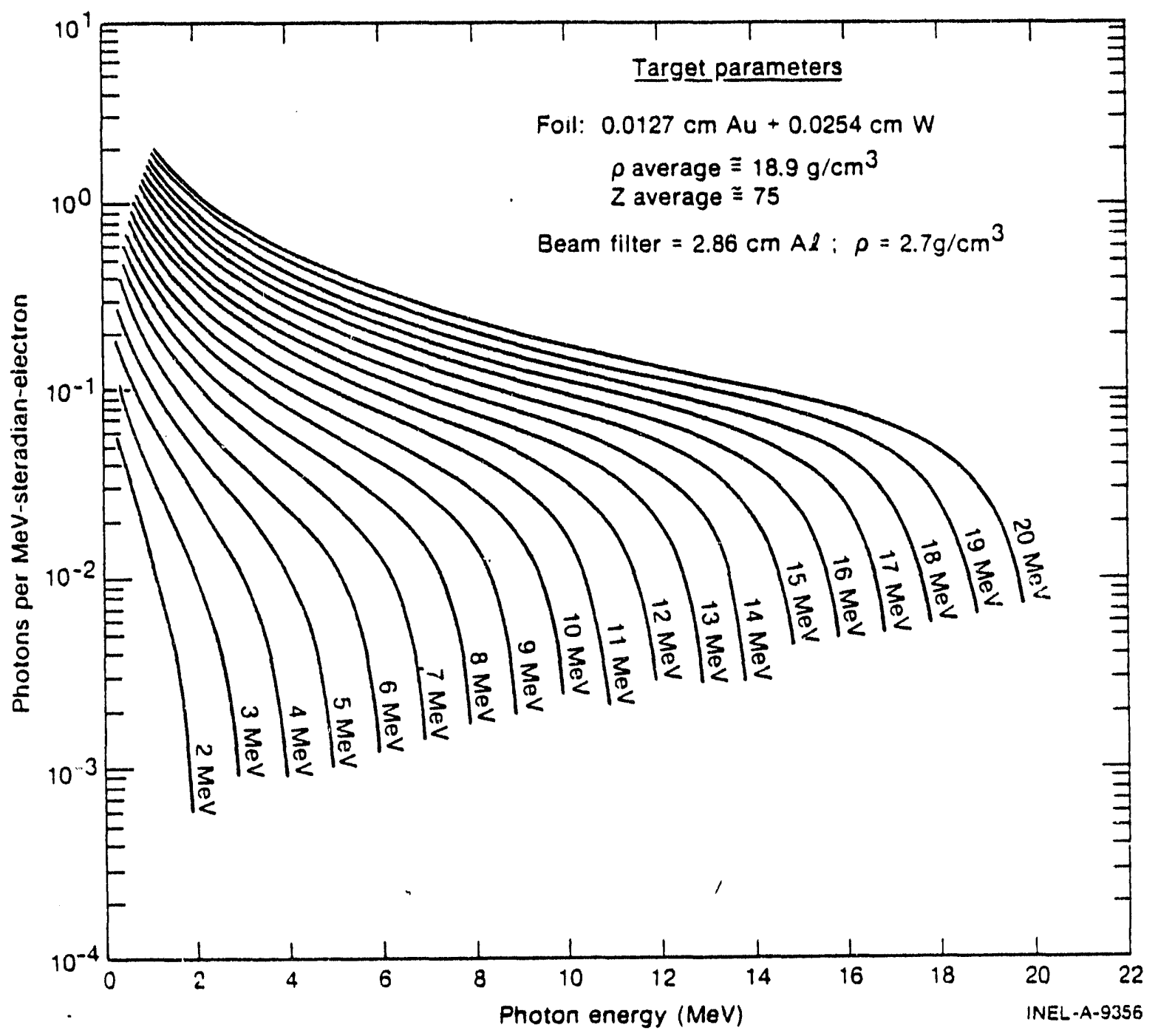

Figure 3.1 Calculated bremsstrahlung X-ray spectra for various electron beam energies. (Reproduced from Reference 1) 

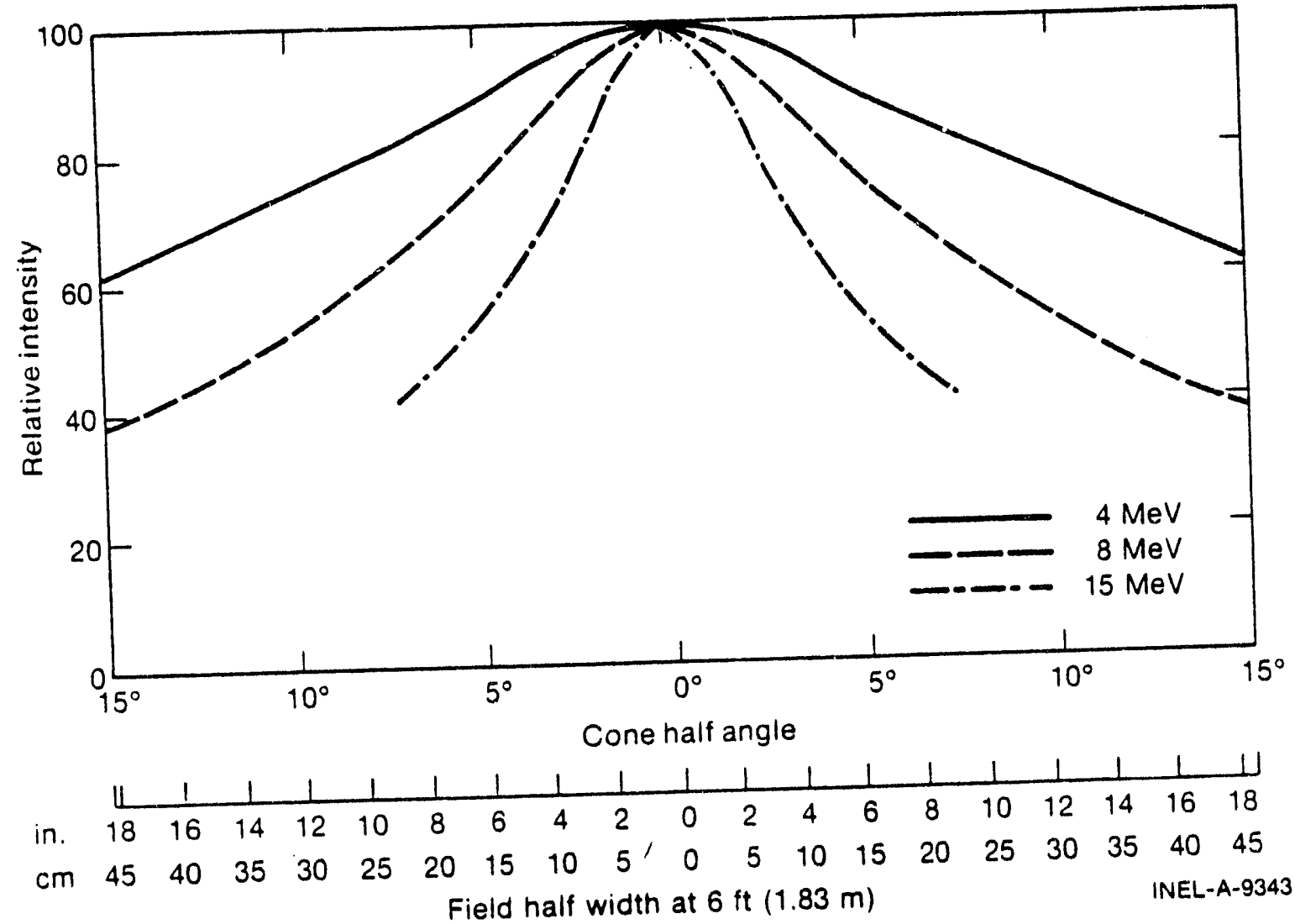

Figure 3.2 X-ray intensity distributions for various electron beam energies. (Reproduced from Reference 1) 


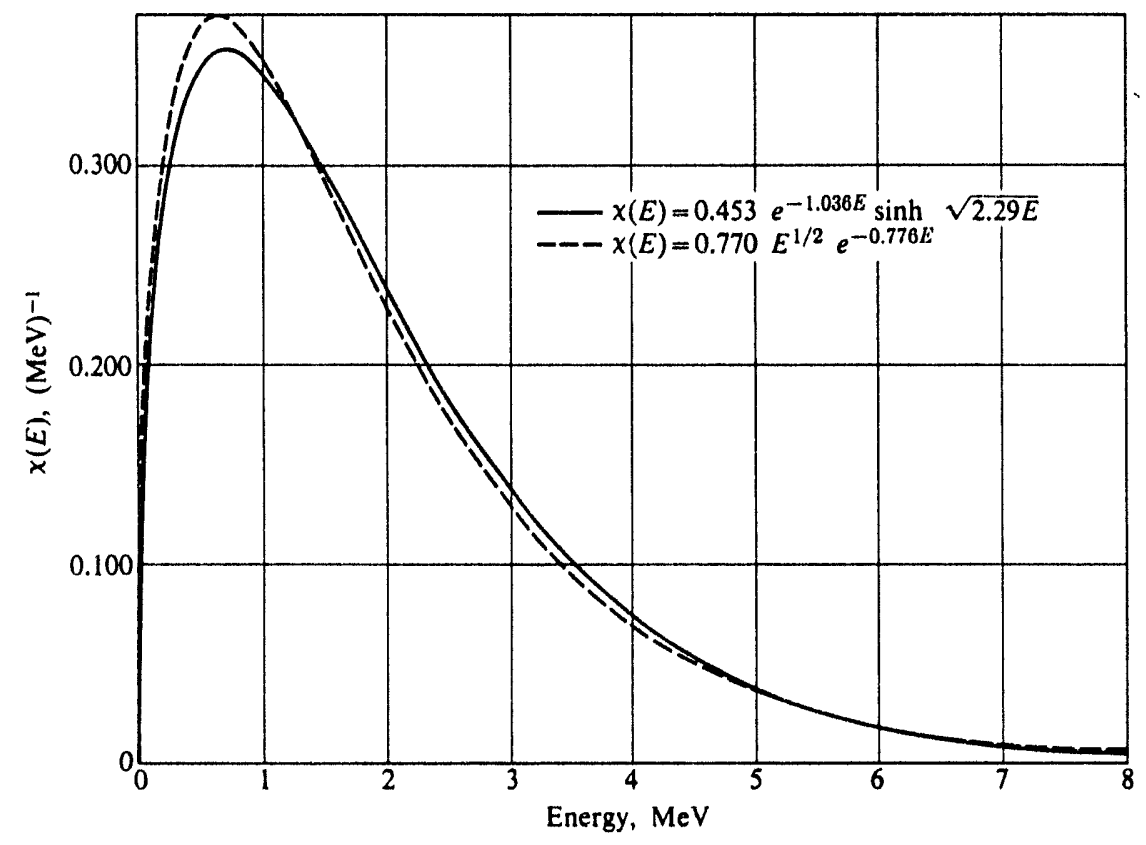

Figure 3.3 The prompt neutron fission spectrum. (Reproduced from Reference 2) 
only in nuclear materials and have a threshold in the range of 5.5 to $7.0-\mathrm{MeV}$. $\mathrm{A}$ representative list ${ }^{7}$ of photoneutron and photofission nuclides are included in Table 3.1 along with their natural abundance and energy thresholds. As can be seen in this table, beryllium has the lowest known photoneutron threshold energy (1.7 MeV) and, as such, it makes a highly desirable photoneutron production medium.

Neutron production within an interrogated object is dependent on the photon flux within the object, the materials within the object, the photoneutron interaction probability of each photon component of the bremsstrahlung distribution, and the volume of the object illuminated. Due to these various parameters, an interrogation configuration must be defined before specific neutron production estimates can be presented. Section 3.2.2 and 4 define and discuss a representative interrogation configuration.

\subsubsection{Fundamental Timing}

The duration of any $X$-ray production is dependent on the pulse width $\left(t_{p}\right)$ of the electron accelerator beam pulses utilized. Once produced, the speed of the photons (X-ray) in air is approximately 3.34 ns per meter. The interaction of the $X$ rays with an interrogated object results in either a reflection (elastic scatter) of the $X$-rays away from the object or an absorption or inelastic scattering interaction. Some of the latter interactions result in the production of photoneutrons or photofission neutrons. The neutron production processes occur so rapidly that they can be considered as instantaneous. Once produced the neutrons either leak from the object or interact in the object. Some of the latter interactions result in the production of elemental characteristic, secondary gamma emissions. While gamma emissions can result as soon as the neutrons are created, the fact that neutrons are slower than the photons, imposes a natural time delay between the reflected (elastic scattered) X-rays and the neutron-induced, secondary gamma emissions. This time delay will be smaller as the neutron energy increases.

To appreciate the timing considerations related to this technology, a simplistic, but representative, overview will be presented which is summarized in Figure 3.4.

Assume a 90-degree $X$-ray source-object-point detector configuration having $1.5-\mathrm{m}$ $X$-ray source-to-object(center) and object(center)-to-detector distances (See Figure 3.5). Further assume a 1-m diameter interrogation object. As shown in Figure 3.2 for an 8-MeV electron beam pulse, the full-width-at-half-maximum of the X-ray intensity distribution is about $50 \mathrm{~cm}$, which means that 50 percent of the object in Figure 3.5 will be effectively illuminated. [Note, this technology does not require complete object illumination.] Assume that the electron beam pulse is approximated by a step impulse initiated at $t=0$. Based on the photon speed, the initially produced $X$-rays arrive directly at the detector in $7.1 \mathrm{~ns}(2.12 \mathrm{~m} \times 3.34$ $\mathrm{ns} / \mathrm{m}$ ). In the object, the neutron production ideally occurs between 3.3 (time for 
TABLE 3.1 REPRESENTATIVE PHOTONEUTRON/PHOTOFISSION PRODUCTION NUCLIDES

\begin{tabular}{|c|c|c|}
\hline Element/lsotopes & $\begin{array}{l}\text { Percent Nat. } \\
\text { Abundance }\end{array}$ & $\begin{array}{c}(y, n) \text { Threshold } \\
(\mathrm{MeV})\end{array}$ \\
\hline \multicolumn{3}{|l|}{ General Nuclei } \\
\hline $\mathrm{H}-2$ & 0.02 & 2.2 \\
\hline Li-6 & 7.5 & 5.6 \\
\hline $\mathrm{Li}-7$ & 92.5 & 7.3 \\
\hline $\mathrm{Be}-9$ & 100 & 1.7 \\
\hline C-12 & 98.89 & 18.7 \\
\hline C-13 & 1.11 & 4.9 \\
\hline$N-14$ & 99.63 & 10.6 \\
\hline$N-15$ & 0.37 & 10.8 \\
\hline 0.16 & 99.76 & 15.7 \\
\hline 0.17 & 0.04 & 4.1 \\
\hline 0.18 & 0.20 & 8.0 \\
\hline \multicolumn{3}{|l|}{ Nuclear Nuclei } \\
\hline Pu-239 & - & $5.6[5.6]^{\circ}$ \\
\hline Pu-240 & - & $6.5[6.1]$ \\
\hline Pu-241 & - & $5.3[5.4]$ \\
\hline U-232 & - & $7.3[6.7]$ \\
\hline U.233 & . & $5.7[6.0]$ \\
\hline$U-235$ & 0.72 & $5.3[5.8]$ \\
\hline U.236 & - & $6.5[5.9]$ \\
\hline$U-238$ & 99.27 & $6.1[5.8]$ \\
\hline Th-232 & 100 & $6.4[6.0]$ \\
\hline \multicolumn{3}{|l|}{ Structural Nuclei } \\
\hline Cu-63 & 69.2 & 10.8 \\
\hline $\mathrm{Cu}-65$ & 30.8 & 9.9 \\
\hline $\mathrm{Fe}-54$ & 91.8 & 11.2 \\
\hline $\mathrm{Fe}-56$ & 2.1 & 7.6 \\
\hline $\mathrm{Fe}-58$ & 0.3 & 10.0 \\
\hline$W-182$ & 26.3 & 8.0 \\
\hline$W-183$ & 14.3 & 6.2 \\
\hline$W-184$ & 30.7 & 7.5 \\
\hline$W-186$ & 28.6 & 7.3 \\
\hline Al- 27 & 100. & 13.1 \\
\hline $\mathrm{Ca}-40$ & 96.94 & 15.7 \\
\hline $\mathrm{Ca}-43$ & 0.14 & 7.9 \\
\hline $\mathrm{Ca}-44$ & 2.09 & 11.1 \\
\hline Si-28 & 92.2 & 17.2 \\
\hline Si-29 & 4.7 & 8.5 \\
\hline Si-30 & 3.1 & 10.6 \\
\hline
\end{tabular}




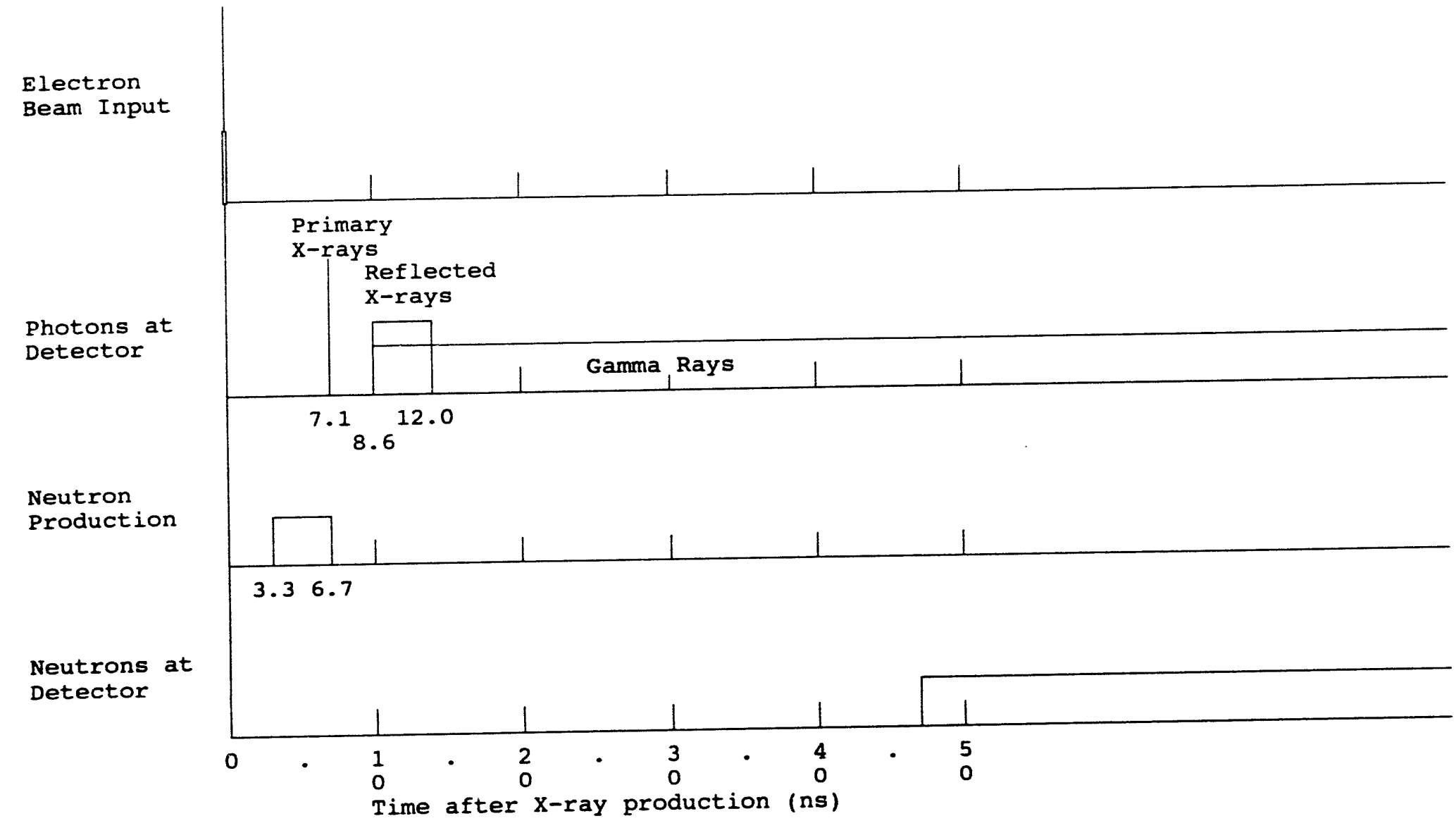

Figure 3.4 General concept timing considerations for $X$-rays, gamma, and neutrons for an input electron beam impulse. 




Figure 3.5 Basic geometry for primary concept components. 
photon to reach surface of object) and $6.7 \mathrm{~ns}$ (maximum time before leakage). In addition, the object-reflected (scattered) $X$-rays will arrive at the detector between 8.6 (travel of $1 \mathrm{~m}$ plus the square root of $1.5^{2}$ and $0.5^{2}$ ) and $12.0 \mathrm{~ns}$. The latter time corresponds to the occurrence of an X-ray scattering event just prior to object leakage (travel of $2 \mathrm{~m}$ plus the square root of $1.5^{2}$ and $0.5^{2}$ ). Hence, for the assumed configuration, two pulses of $X$-rays arrive at the detector; the first is the intense interrogating $X$-rays at about 7.1 ns and the second is the intensityreduced reilected $X$-rays beginning at approximately $8.6 \mathrm{~ns}$.

Now consider the resulting photoneutrons. These neutrons are created with a continuous-energy spectrum below a maximum energy. Assume the continuous neutron energy spectrum is represented by two neutron energies; i.e., 6.0 and $0.05-\mathrm{MeV}$. Table 3.2 presents selected neutron energy and speed relationships. For this spectrum approximation, the neutrons leaking from the object will arrive at the detector as soon as 47.6 or as long as $488 \mathrm{~ns}(1.5-\mathrm{m}$ average travel time for an instantly leaked 6 and $0.5-\mathrm{MeV}$ neutrons, respectively). Since neutron interactions with the object material can occur immediately after neutron production, secondary gamma emissions will arrive at the detector as soon as 8.6 ns. As can be seen, a time window exists between 12.0 and 47.6 ns in which the detector is only exposed to characteristic gamma emissions. Striving to obtain gamma detection within this nominal 36-ns window is very desirable since the response will be dominated by the rapidly occurring, short-lived, neutron inelastic events which provide additional elemental object information than conventional longer time response dominated by neutron capture events.

TABLE 3.2 SELECTED NEUTRON ENERGY AND CORRESPONDING SPEED ${ }^{8}$

$\begin{array}{ccc}\begin{array}{c}\text { Energy } \\ \text { (MeV) }\end{array} & \begin{array}{c}\text { Speed } \\ (\mathrm{m} / \mathrm{s})\end{array} & \begin{array}{r}\text { Time/meter } \\ (\mathrm{ns} / \mathrm{m})\end{array} \\ 16.0 & 5.69 \mathrm{E} 7 & 17.6 \\ 10.0 & 4.37 \mathrm{E} 7 & 22.9 \\ 8.0 & 3.91 \mathrm{E} 7 & 25.6 \\ 6.0 & 3.39 \mathrm{E} 7 & 29.5 \\ 4.0 & 2.77 \mathrm{E} 7 & 36.2 \\ 2.0 & 1.96 \mathrm{E} 7 & 51.1 \\ 1.0 & 1.38 \mathrm{E} 7 & 72.3 \\ 0.5 & 9.78 \mathrm{e} 6 & 102.0 \\ 0.05 & 3.09 \mathrm{e} 6 & 323.0 \\ 1 . \mathrm{E}-6 & 1.38 \mathrm{E} 4 & 72300.0\end{array}$


Despite the simplistic approach and assumptions used above, the fundamental description does provide a representative overview of the key temporal responses related to this concept. Also, the above responses can be interpreted with finite electron beam pulse widths. Any increase in the electron beam pulse width will correspondingly increase the primary $X$-ray response and extend the scattered $X$ ray and the neutror: production response times (See Figure 3.4). Therefore, for a given component configuration the gamma emission detection window becomes more constricted with increasing electron beam pulse widths. For detection of neutron-induced inelastic gamma emissions, the electron pulse width must be as small as possible and at least less than the characteristic neutron production time defined by the spacial extent of the interrogated object. X-ray shielding around the detector(s) will be important for minimizing the detector recovery time, especially after exposure to the primary, intense $X$-ray interaction (i.e., flash). For the detection of neutron-induced capture gamma emissions at longer times (>500 ns), the requirement on electron pulse width becomes more relaxed and neutron shielding around the detector(s) will become more important to minimize signal-tonoise effects.

\subsection{Accelerator Technology}

The electron accelerator will generate pulses of electrons. The repetition rate of these pulses define the maximum time available for gamma detection (i.e., $100 \mathrm{~Hz}$ corresponds to a time between pulses of $10 \mathrm{~ms}$ ). For each pulse, the primary $X$ ray (Dose) emission/production rates $^{9}$, as shown in Figure 3.6, is a product of the electron beam energy and current (i.e., amplitude and pulse width). While the current component is a linear component to the total forward-directed emission rates (dose), the beam energy is nonlinear (i.e., proportional to $E^{2.7}$ ). The reflected $X$-ray emissions from the interrogated object will be dependent on the object geometry and material composition, and it will be proportional to the primary $X$-ray production. Clearly, a minimum dose to the object and the environment is desirable.

The performance of currently available electron accelerator technologies varies considerably. Compact, modular, transportable, electron accelerators are commercially available from various vendors (Varian Inc., High Energy Services Company (HESCO), Schonberg Radiation Corporation). These commercial accelerators produce electron beam energies (up to $15 \mathrm{MeV}$ ), peak beam current in excess of $500 \mathrm{~mA}$, and pulse widths up to 5 microseconds (i.e., corresponds to greater than $10^{13}$ electrons/pulse). These accelerators can operate at repetition rates of up to $1 \mathrm{kHz}$. Typically, these accelerators are used for commercial radiography requiring high dose rates. At DOE laboratories, higher energy and current accelerators are available, such as the EG\&G Energy Measurements Linear Accelerator Facility, which will produce up to $25-\mathrm{MeV}$ electrons, at up to $40 \mathrm{~A}$, with accelerator pulse widths of 50 picoseconds at $20 \mathrm{~Hz}$ (Per pulse, this 


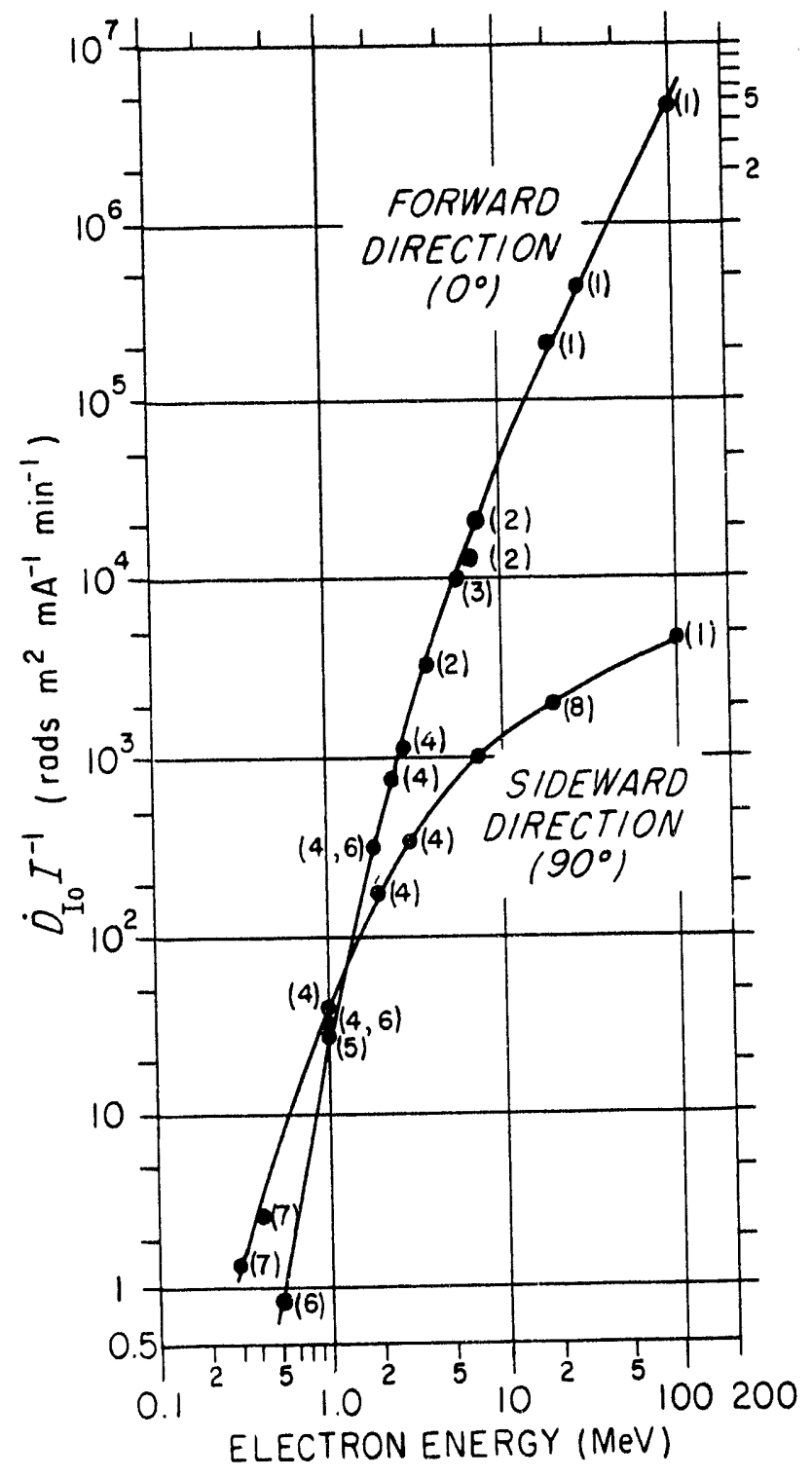

Figure 3.6 X-ray emission rates from high- $Z$ convertors (targets). (Reproduced from Reference 9) 
corresponds to $2.5 \times 10^{10}$ electrons). For the MIT concept which attempts to maximize the neutron production and minimize dose rate, it is anticipated a high repetition rate, moderate intensity beam current $(<10 \mathrm{~mA}$ peak), and a short pulse width $1<50 \mathrm{~ns}$ for inelastic neutron scattering response or a greater than $500 \mathrm{~ns}$ pulse width for neutron capture) response will be required. The short pulse width minimizes dose on target and reduces adverse effects on the detectors (i.e., recovery time). The high repetition rate enables short interrogation times. These accelerator characteristics are not technologically challenging and actually drive typical accelerators to minimal performance requirements. In most cases, the implementation of these characteristics require relatively minor modifications of existing pulse forming networks (PFN) supplying power to the accelerators.

\subsection{Candidate Fast Detectors}

The basic detection timing requirements for the induced neutrons and secondary gamma emissions have been discussed in Section 3.2.2 and highlighted in Figure 3.4. Clearly two detection regimes exist: 1) an ultra-fast regime capable of pulse amplitude detection of inelastic neutron scattering events exposed to a minimal neutron flux and 2) a more conventional (slower) regime capable of gamma detection with a coexisting neutron flux. This report focuses on the ultra-fast timing regime.

(1) rapidly recover from both the initial, primary, intense $X$-ray interactions (i.e., flash) and the elastic scattered (reflected) X-rays to enable gamma emission differentiation, and

(2) provide gamma interaction pulses whose pulse height is proportional to the energy of the gamma events.

An ideal detector is one which is neutron insensitive, has a fast decay time of less than $10 \mathrm{~ns}$, has a dominate fast component decay time, has a good energy resolution, and has a high intrinsic efficiency.

Using ultra-fast time response as the primary and most challenging characteristic for assessing this concept, four candidate detectors were identified ${ }^{12}$ : two inorganic materials -- barium fluoride $\left(\mathrm{BaF}_{2}\right)$ and a pure cesium iodide (Csl) from the Bicron Corporation, and two organic (plastic) materials - Bicron Corporation's BC418 and BHTP [4-bromo-4"-(5-hexadecyl)-p-terphenyl] in polystryrene developed ${ }^{10,11}$ by EG\&G Energy Measurements, Inc. While these materials are expected to be representative of the MiT application, they are not a complete set of all applicable fast detectors. The two inorganic materials were assessed because they exhibited a very fast scintillation component (less than $10 \mathrm{~ns}$ "1/e" decay time) and still retain some photoelectric efficiency at high energies. The 
plastic scintillators were assessed because all of the scintillation light is emitted in a single fast (less than $2.5 \mathrm{~ns}$ "1/e" decay time) component. BC-418 and BHTP do not provide direct full energy information because their photoelectric cross sections are negligible. However, spectrum unfolding of their Compton response will yield full energy information.

These detectors require opto-electronic conversion devices to produce electronic pulses for processing and analysis. Pure $\mathrm{CsI}_{1} \mathrm{BaF}_{2}$, and $\mathrm{BHTP}$ light emission is in the 250-300 nanometer range and require a quartz window photomultiplier tube (PMT) for conversion. BC-418 light emission is in the $450 \mathrm{~nm}$ range and requires a standard window (borosilicate) PMT. The PMTs used in this assessment were Hamumatsu Type-H3378, which are advertised to have a 0.8 ns rise time, 3 ns decay time, and 1.5 ns half-height response.

The inorganic crystals investigated were a 1 -inch diameter by 2 -inch long (i.e., 1 " $\times 2$ ") $\mathrm{BaF}_{2}$ and a $1 " \times 1$ " and a 2" $\times 2$ " pure Csl. The plastic scintillators were $2 " \times 2 " \mathrm{BC}-418$ and BHTP. The $\mathrm{BaF}_{2}$ exhibited a fast scintillation component of less than 1 ns decay time and a slow component of 620 ns decay time. However, eighty percent of the light is in the long decay component. Assessments showed that while $\mathrm{BaF}_{2}$ had an extremely fast rise time, its recovery time was too long for practical consideration for this concept. Pure Csl exhibited a fast scintillation component with 6-8 ns decay time and a slow scintillation decay component with 25-35 ns. Greater than $88 \%$ of the Csl scintillation light is in the desirable fast component. Bicron continues to investigate additional enhancements to this detector material.

A fast pulse stretcher circuit ${ }^{13}$ was designed and built to allow storage of the peak value of the fast component pulses from these detectors. Using the fast pulse stretcher circuit, the energy resolution of these crystals were estimated using standard analog-to-digital converters (ADCs) and laboratory radioactive sources. These measurements are included in Table 3.3. The results suggest that pure CsI and BHTP are resolution limited by self-absorption of their own scintillation light. Hence, application of these materials for this interrogation concept implies smaller (i.e., 1" $\times 1$ ") sized-detectors. The BHTP and 2" $\times 2$ " Csl energy resolution for the energy region of interest for this application (above $1.5-\mathrm{MeV}$ ) appears to be too large. The $\mathrm{BC}-418$ and the 1 " $\times 1$ " Csl energy resolution appear suitable for this concept. Final energy resolution assessments will depend on the final fast detection system design. 


\section{TABLE 3.3 DETECTOR FAST COMPONENT ENERGY RESOLUTION}

Detector Material Energy Resolution

\begin{tabular}{|c|c|c|c|c|}
\hline $\begin{array}{l}\text { Energy } \\
\text { (keV) }\end{array}$ & $\begin{array}{c}\mathrm{Cs}(1 \times 1) \\
(\mathrm{keV})\end{array}$ & $\begin{array}{c}\text { Csl }(2 \times 2) \\
(\mathrm{keV})\end{array}$ & $\begin{array}{c}\text { BHTP }(2 \times 2) \\
(\mathrm{keV})^{:}\end{array}$ & $\begin{array}{c}\mathrm{BC}-418(2 \times 2) \\
(\mathrm{keV})^{\mathrm{a}} \\
\end{array}$ \\
\hline $477^{b}$ & - & - & 172 & 143 \\
\hline $661^{c}$ & 152 & 205 & - & - \\
\hline $1038^{d}$ & - & - & 270 & 166 \\
\hline $1250^{\circ}$ & 438 & 338 & - & - \\
\hline $2381^{f}$ & - & - & 452 & 214 \\
\hline $2614^{g}$ & 209 & 444 & - & - \\
\hline
\end{tabular}

a - value taken from 0.75 and 0.25 of maximum of Compton edge

b - ${ }^{137}$ Cs Compton edge

c - ${ }^{137}$ Cs photopeak

d $-{ }^{60}$ Co Compton edge

$\mathrm{e}$ - average of ${ }^{60} \mathrm{Co} 1332 \mathrm{keV}$ and $1173 \mathrm{keV}$ energies

f - ${ }^{228}$ Th Compton edge

g- ${ }^{228}$ Th photopeak 


\section{NUMERICAL PREDICTIONS}

Several numerical predictions were performed to assess the Material Identification Technology concept. These assessments involved a prescribed calculational methodology and a representative model including several typical object materials of interest. Numerical response characterizations were performed for two electron beam energies and two general system configurations.

\subsection{Methodology}

The numerical methodology, presented in Figure 4.1, has been used to predict neutron, photon, and secondary gamma response for representative interrogation configurations. The methodology predicts electron-photon interaction and transport using the ACCEPT code ${ }^{14}$, calculates photoneutron production associated with an interrogated object using an INEL developed code (PINP; see listing in Appendix), and neutron interaction and transport to the detector using the MCNP4 code ${ }^{15}$. For each case assessment, an initial electron-photon interaction calculation was made to identify the photon flux in all regions of a defined system model. Based on these photon fluxes, a calculated photoneutron production spectra was used as the neutron source spectra for the neutron transport code. This coupling resulted in a "step impulse" source neutrons insertion for the final neutron transport/interaction modeling.

\subsection{Model Description}

Figure 4.2 shows the calculation model used in these representative predictions. The specific model was chosen to represent large object interrogations such as those representative of export/customs inspections. As shown, the model consists of a thin convertor to generate the energetic X-rays, a spherical object for interrogation, and a point gamma and neutron detector. All three components are centered in a horizontal plane. The $\mathrm{X}$-rays are generated from the interaction of a $0.1-\mathrm{cm}$ radius electron beam with a $0.1-\mathrm{cm}$ thick tungsten convertor. The electron beam direction is normal to the surface of the tungsten convertor which is large compared to the beam diameter. The selected thickness of the tungsten convertor represents a thin target relative to the 8- and 13- MeV applied electron beam energies. The interrogated object assumes the form of a sphere of differing radii, containing differing materials, and is surrounded by a $0.5-\mathrm{cm}$ thick beryllium shell. The beryllium provides the primary source of neutrons and the interrogated object will, depending on the electron beam energy, provide additional source neutrons. For these conservative calculations, only beryllium produced neutrons are modelled. The distance between the convertor and the object surface was always maintained at 1 meter regardless of the radius of the object. A gamma/neutron 


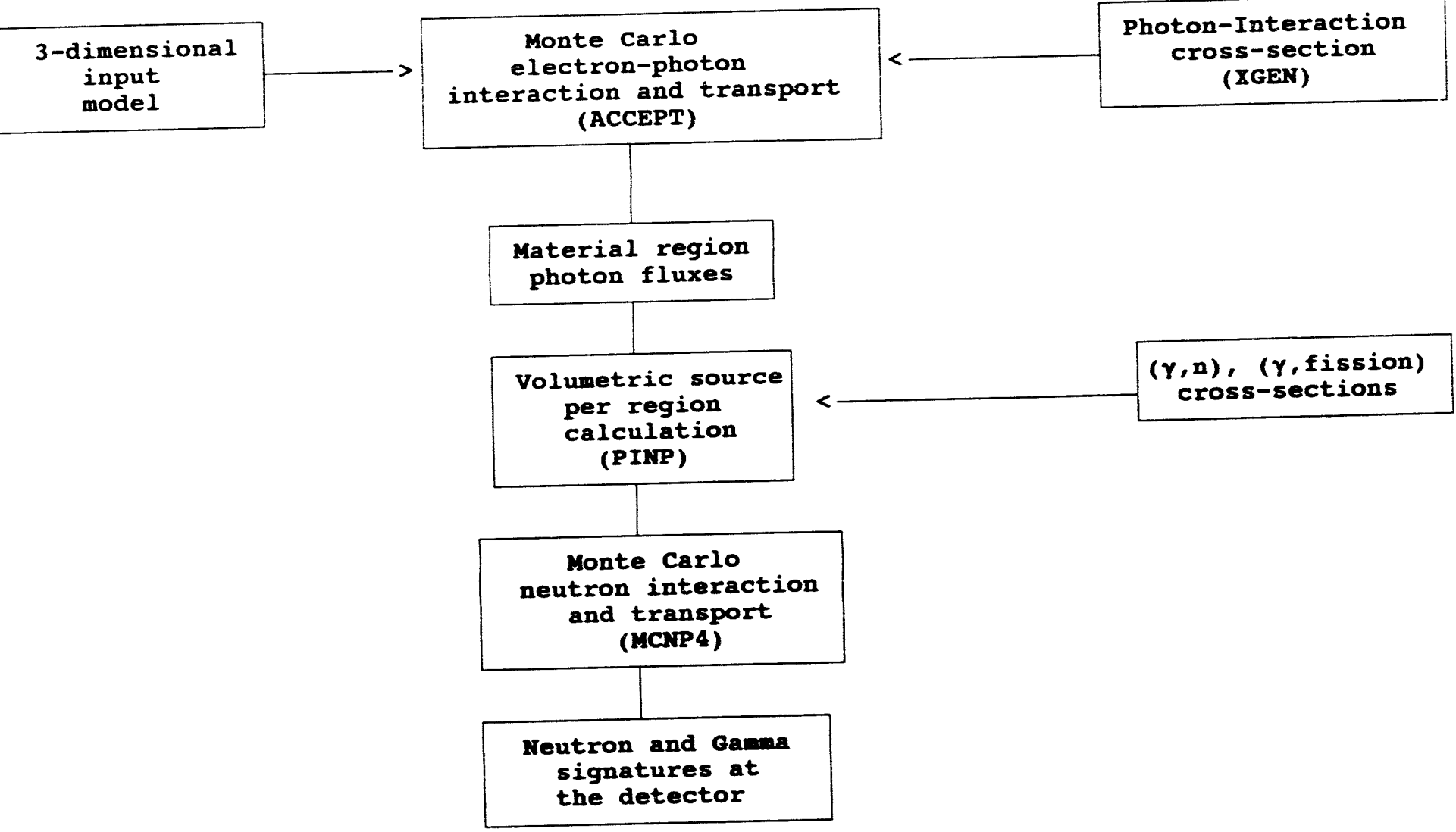

Figure 4.1 Numerical Modeling Methodology 


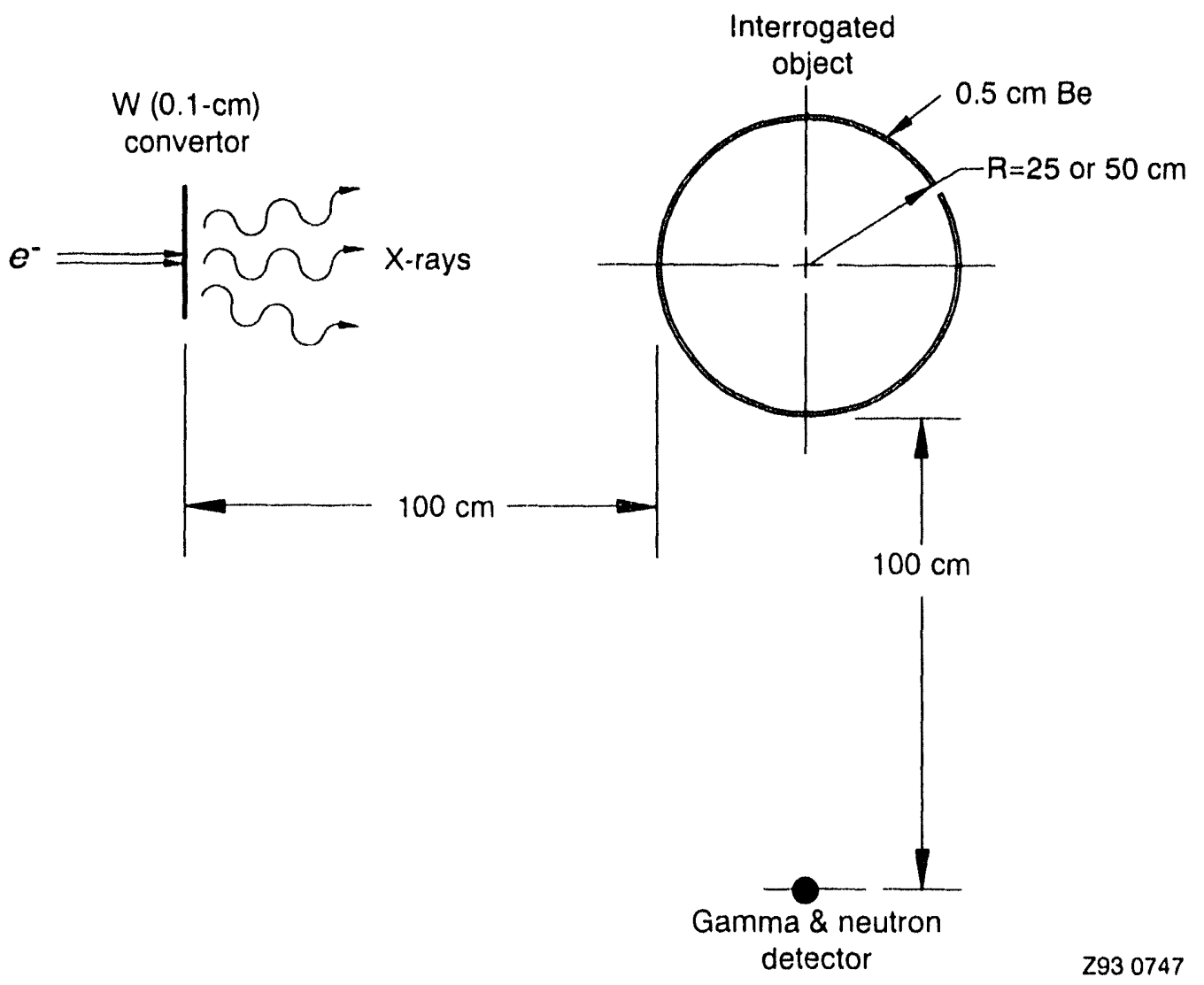

Figure 4.2 Calculational model. 
detector is positioned at a right angle to the electron beam centerline and the center of the object sphere. A constant object surface-to-detector distance of 1 meter is maintained.

\subsection{Interrogated Objects Description}

Three object material compositions were considered in this assessment: a chemical weapon (mustard gas ${ }^{16}-1.9 \mathrm{~g} / \mathrm{cc}$ ), concrete (NBS-standard ${ }^{17}-2.3 \mathrm{~g} / \mathrm{cc}$ ), and an iron (natural Fe - $7.8 \mathrm{~g} / \mathrm{cc}$ )/polyethylene (Poly - $0.9 \mathrm{~g} / \mathrm{cc}$ ) composite . Specific material compositions are included in Table 4.1 for each interrogated object modeled. For the first two object compositions, two configurations are considered: outer radius (i.e., inner beryllium shell radius), $R_{0}$, of $25-\mathrm{cm}$ and $50-\mathrm{cm}$. The third object material configuration is fixed with $R_{o}^{{ }^{F e}}=45 \mathrm{~cm}$ and $R_{o}{ }^{\text {Poly }}=50 \mathrm{~cm}$.

\subsection{Results}

Table 4.2 provides a summary of the cases assessed and includes the corresponding neutron production in the beryllium shell $\left(S_{n}\right)$ and total neutron and gamma fluence $\left(\# / \mathrm{cm}^{2} / \mathrm{S}_{\mathrm{n}}\right)$ at the point detector location. As can be seen, for the cases studied, the ratio of the total neutron-to-gamma fluence at the detector is about a factor of ten. This type of neutron sensitivity must be included in the final system design (i.e., shielding and/or detector selection). Based on Case 1 shown in Table 4.2, the current electron accelerator technology (See Section 3.3) of about $10^{12}$ electrons/pulse, and a $2.54-\mathrm{cm}$ diameter detector, results in approximately 71 photon events at the detector. Assuming a $5 \%$ intrinsic efficiency, this results in about 3-4 detected events per accelerator pulse. If all detected events can be processed, an accelerator operating at $1 \mathrm{kHz}$ could acquire up to 4000 counts per second for the interrogated object in Case 1.

\subsubsection{Neutron Production}

Figure 4.3 presents the total neutron production capacity of the $0.5-\mathrm{cm}$ thick beryllium shell with an inside radius of $50-\mathrm{cm}$ for various electron beam energies. For these calculations, air has replaced the object material(s) within the shell. By comparison to the included neutron production values of Table 4.3 (Cases 1 through 3 , and 5 through 71 , it is apparent that most of the neutron production (greater than $95 \%$ ) occurs on the hemispherical shell directly facing the $X$-ray source. Hence, Table 4.3 is also applicable to other object materials and supports the concept that a wall (sheet) of beryllium, near the object and directly between the $X$-ray source and the object is a reasonable geometry. Figure 4.3 also includes the average energy of the total photoneutron production. However, as shown in Figure 4.4 , the neutron production spectra result in a high energy $(>1.5 \mathrm{MeV})$ 


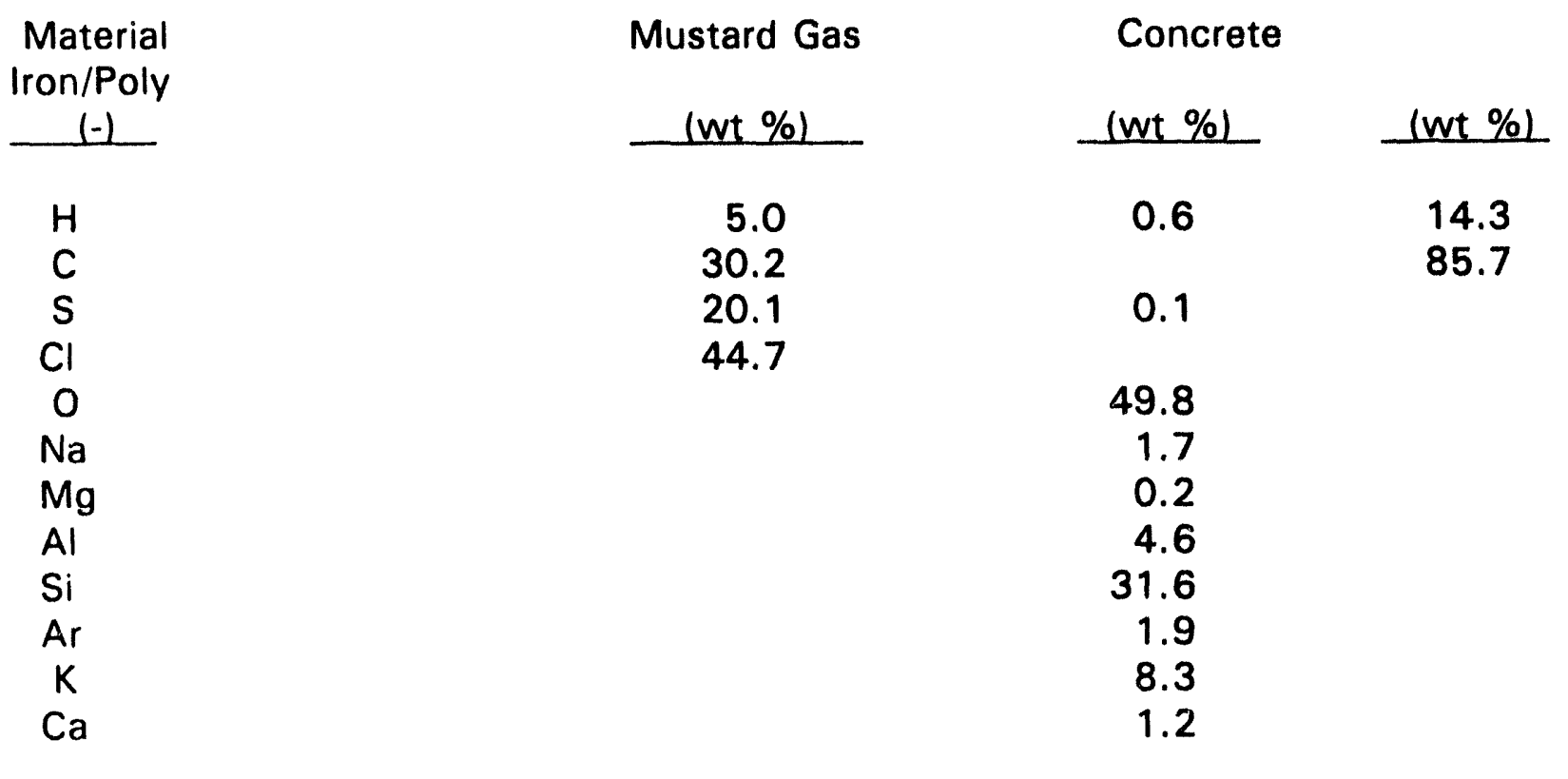

TABLE 4.2 SELECTED RESULTS FOR VARIOUS CASES ASSESSED

\begin{tabular}{|c|c|c|c|c|c|c|}
\hline \multirow[b]{2}{*}{ Case } & \multirow[b]{2}{*}{ Material } & \multirow[b]{2}{*}{$\begin{array}{c}\text { Electron } \\
\text { Energy } \\
(\mathrm{MeV}) \\
\end{array}$} & \multirow[b]{2}{*}{$\begin{array}{l}\text { Sphere } \\
\text { Radius } \\
\text { (cm) }\end{array}$} & \multirow[b]{2}{*}{$\begin{array}{l}\text { Neutron } \\
\text { Production } \\
\quad\left(\mathbf{S}_{n} / \mathrm{e}\right) \\
\end{array}$} & \multicolumn{2}{|c|}{$\begin{array}{c}\text { Fluence at the } \\
\text { Detector Location }\end{array}$} \\
\hline & & & & & $\begin{array}{l}\text { Neutron } \\
\left\lfloor \# / \mathrm{cm}^{2} / S_{n}\right\rfloor\end{array}$ & $\begin{array}{l}\text { Photon } \\
\left(\# / \mathrm{cm}^{2} / \mathrm{S}_{n}\right)\end{array}$ \\
\hline 1 & MG & 13 & 50 & 1.39E-5 & $2.71 E-6$ & $1.00 \mathrm{E}-6$ \\
\hline 2 & Conc & 13 & 50 & $1.38 E-5$ & $3.41 E-6$ & $2.75 E-6$ \\
\hline 3 & $\mathrm{Fe} / \mathrm{P}$ & 13 & $45 / 50$ & $1.42 E-5$ & $2.98 E-6$ & $5.73 E-7$ \\
\hline 4 & MG & 13 & 25 & 7.17E-6 & $2.77 E-6$ & $1.07 E-6$ \\
\hline 5 & MG & 8 & 50 & 4.49E-6 & $2.68 E-6$ & $9.88 E-7$ \\
\hline 6 & Conc & 8 & 50 & 4.44E-6 & 3.30E-6 & 2.07E-7 \\
\hline 7 & $\mathrm{Fe} / \mathrm{P}$ & 8 & $45 / 50$ & 4.77E-6 & $2.94 E-6$ & $5.25 E-7$ \\
\hline 8 & MG & 8 & 25 & $2.04 \mathrm{E}-6$ & $2.73 E-6$ & $1.05 E-6$ \\
\hline \multicolumn{7}{|c|}{$\begin{array}{l}\overline{M G ~-~ M u s t a r d ~ G a s ~} \\
\text { Conc - Concrete } \\
\text { Fe/P - Iron and Polyethylene }\end{array}$} \\
\hline
\end{tabular}




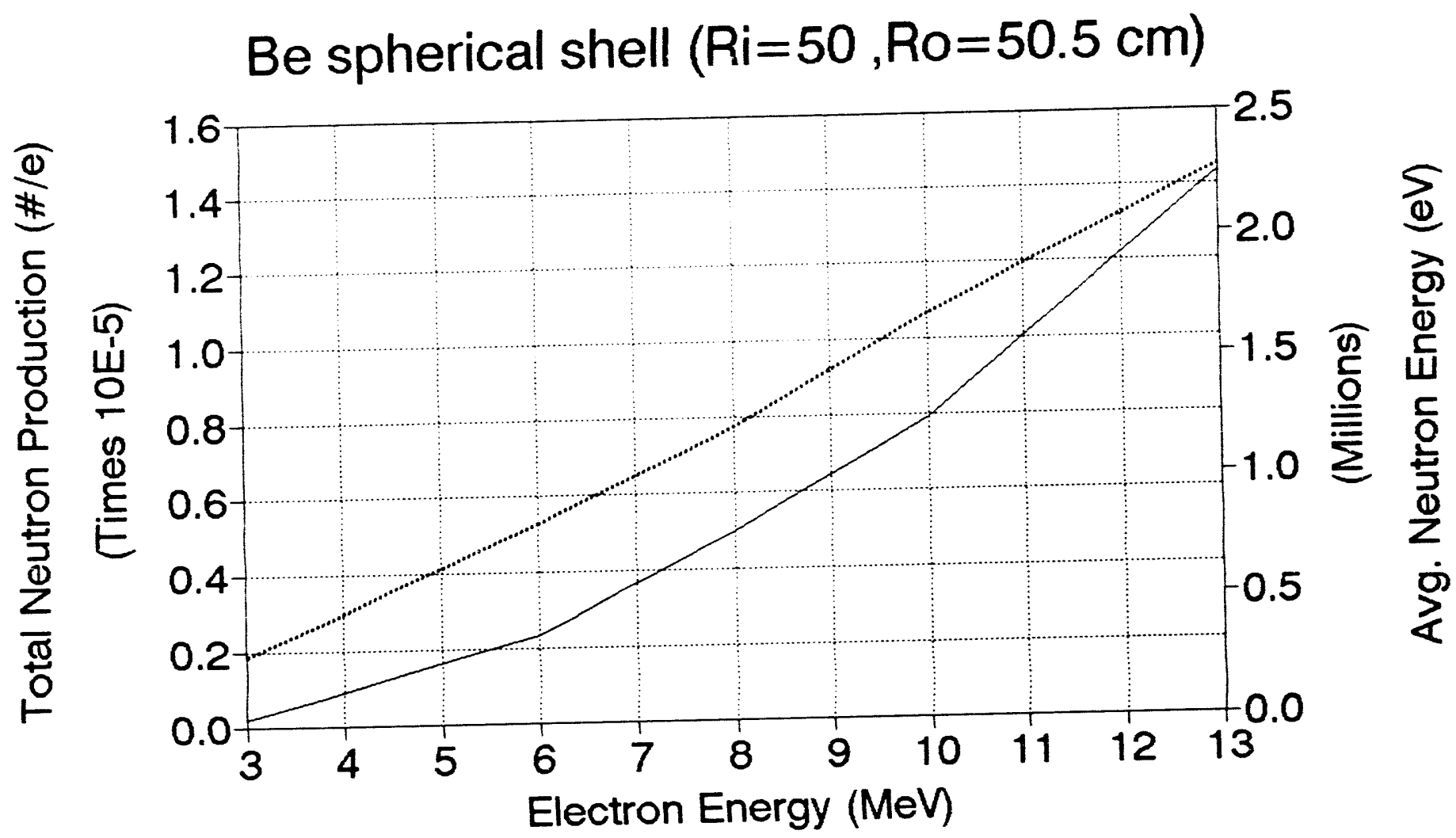

Production

Average Energy energy for the $0.5-\mathrm{cm}$ thick beryllium shell $(R o=50.5 \mathrm{~cm})$ in the calculational model. 
Be spherical shell $(R i=50, R o=50.5 \mathrm{~cm})$

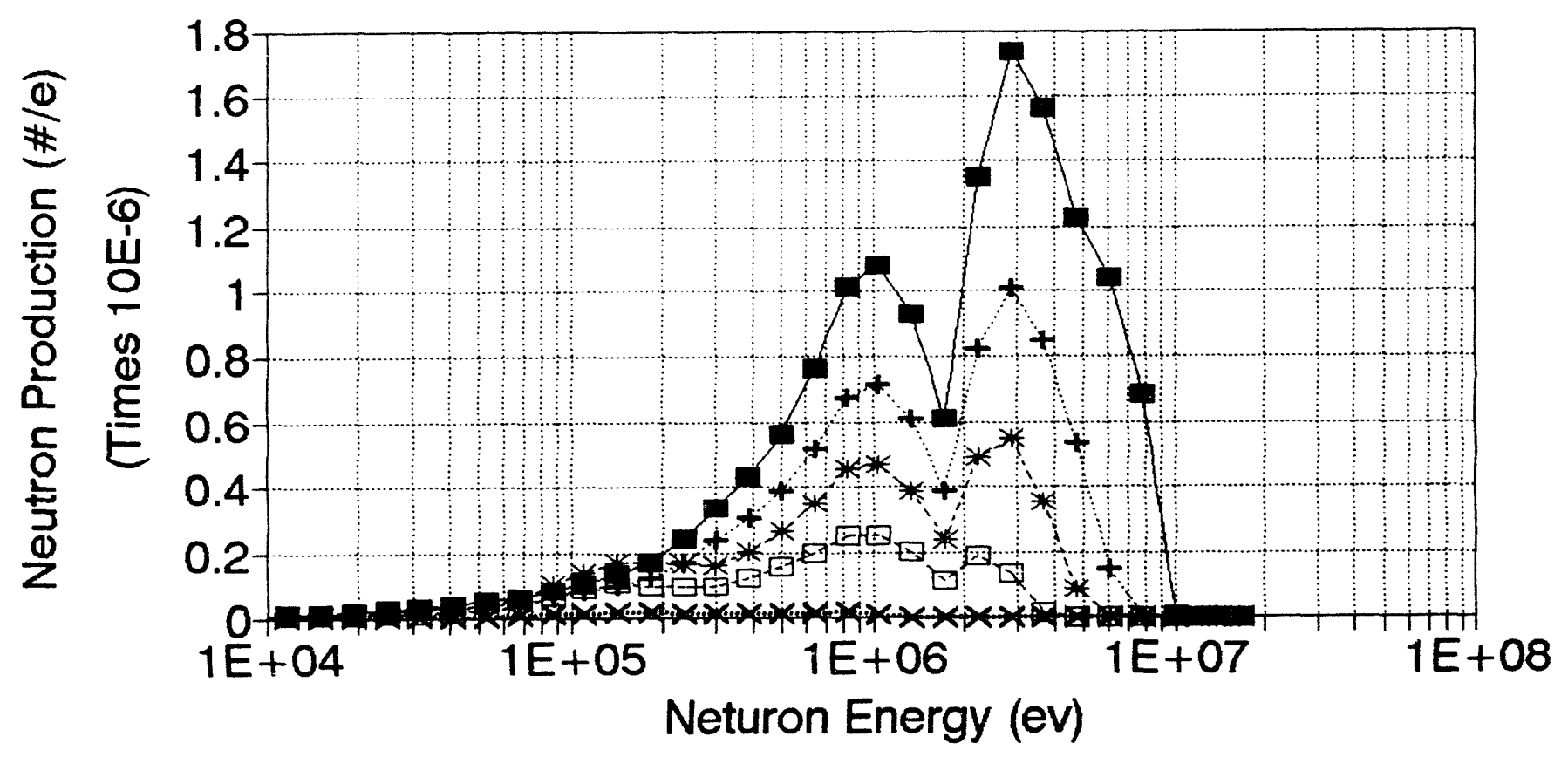

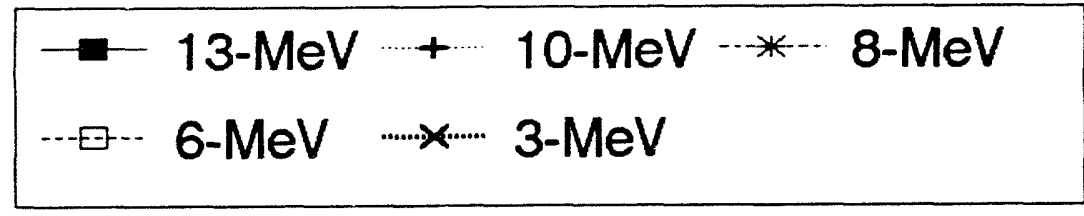
for various electron beam energies. 
contribution and a low energy ( $<1.5 \mathrm{MeV}$ ) contribution. For electron beam energies greater than $3-\mathrm{MeV}$, the resulting neutron production is approximately 50 percent in each category.

\subsubsection{Neutron Response at the Detector}

Figure 4.5 shows the temporal response of the total neutron flux at the point detector for Cases 1 through 3 (see Table 4.2). The time response corresponds to the time after neutron production. A maximum occurs at approximately 50 nanoseconds and falls off by over two orders of magnitude within one microsecond. For the object containing mustard gas, Case 1, the corresponding neutron flux spectra at different time regions after the initial neutron production are presented in Figure 4.6. The rapid progression toward lower total neutron energy content is observed, and most of the neutrons have energies of less than $0.1 \mathrm{MeV}$ energy after $500 \mathrm{~ns}$. Similar overall neutron responses are expected from various electron beam energies and object sizes as seen in the mustard gas comparisons of Cases 1, 4, 5, and 8 shown in Figure 4.7.

\subsubsection{Gamma Response at the Detector}

The total gamma flux temporal responses at the detector after neutron production for the 13-MeV electron cases, Cases 1-3, and the 8-MeV cases, Cases 5-7; are shown in Figures 4.8 and 4.9 , respectively. As a result of the applied calculational methodology described in Section 4.4.1, these figures present only the desired material characteristic, secondary gamma response and excludes any response from the primary $X$-rays. All responses show peaks at less than 10 ns las compared to the $5 \mathrm{~ns}$ required for a photon to traverse the average $1.5 \mathrm{-m}$ distance between the $0.5-\mathrm{m}$ radius object and detector) and then rapidly drop (at least two orders of magnitude) to a level response within about 50 ns. The peak responses of the mustard gas, concrete, and iron/poly materials appear be delayed in time, respectively. This is especially noticeable for the $8-\mathrm{MeV}$ cases shown in Figure 4.9. For the 13-MeV electron energy cases, the common structural materials, such as concrete and iron, show peak and long-term response levels which are at least one order of magnitude lower than the object containing mustard gas. Comparing the mustard gas 13-MeV peak response to its $8-\mathrm{MeV}$ peak response suggests the difference is primarily due increased inelastic neutron scattering events with increased energy.

Figures 4.10 through 4.17 present the detector location spectral gamma responses at various times and for various cases involving the 13-MeV elcctrons, and Figures 4.18 through 4.25 involve the $8-\mathrm{MeV}$ electrons. Two figures are associated with each case assessed. The first shows selected fast time (up to $15 \mathrm{~ns}$ ) spectral gamma responses and the second shows responses in the range of 80 to $5000 \mathrm{~ns}$. Table 4.3 shows correspondence between the figures and the cases assessed. 


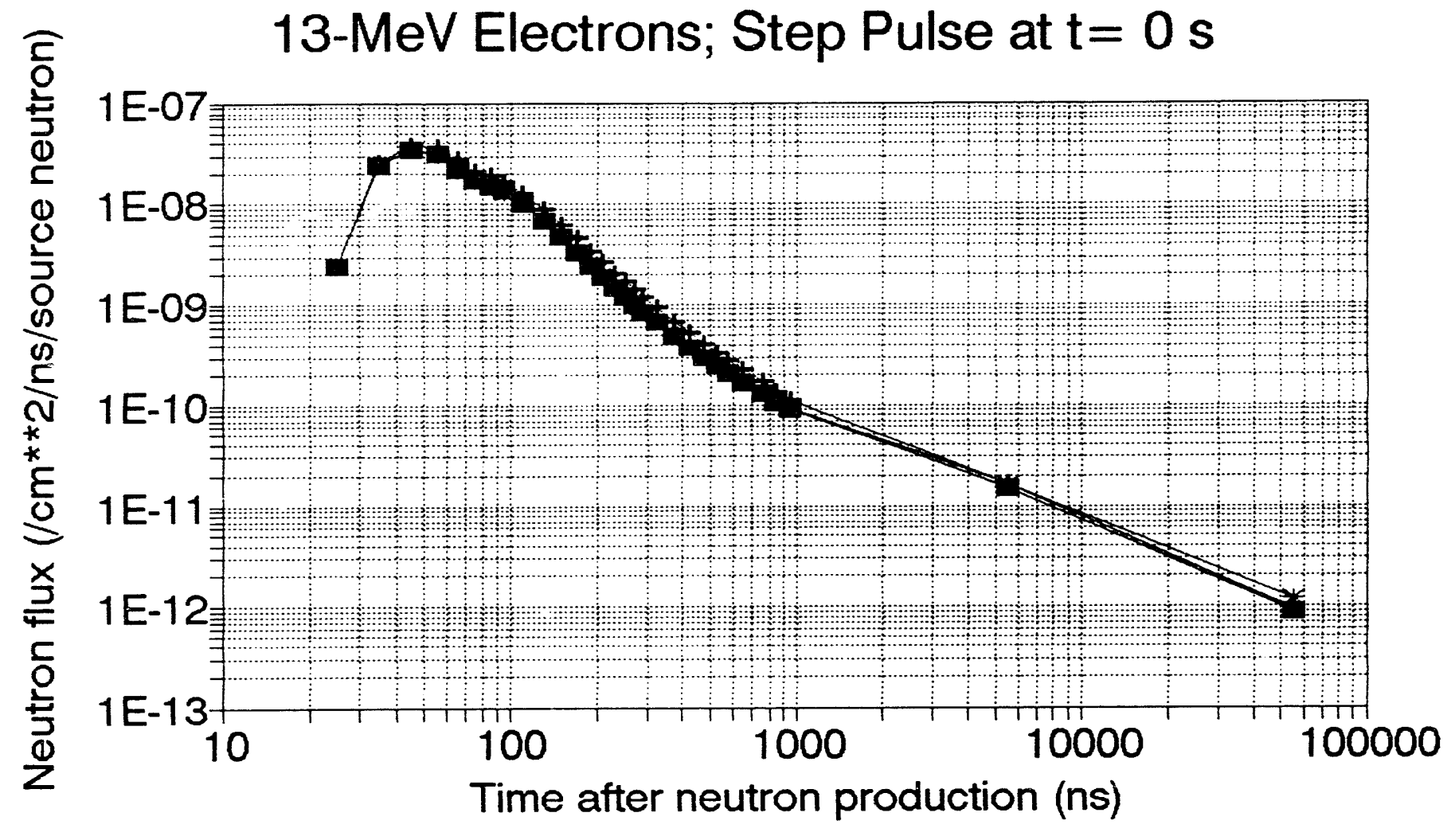

$\stackrel{\sim}{\perp}$

Figure 4.5 Detector location temporal neutron response for various object materials and using 13-MeV electrons (i.e., Cases 1-3). 

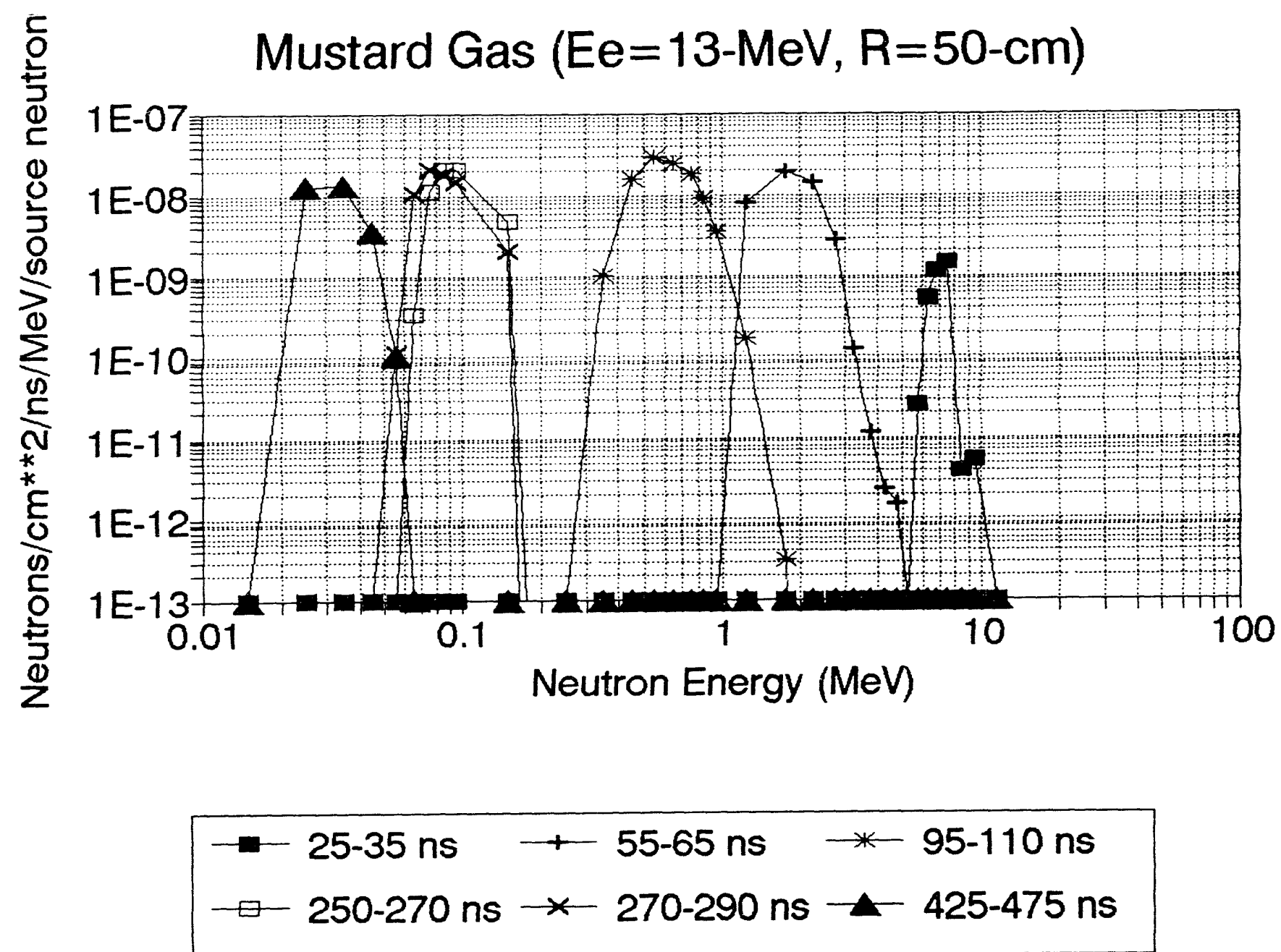

Figure 4.6 Detector location neutron spectral responses for 13-MeV electrons and mustard gas (Case 1). 


\section{Mustard Gas; Step Pulse at $\mathrm{t}=0 \mathrm{~s}$}

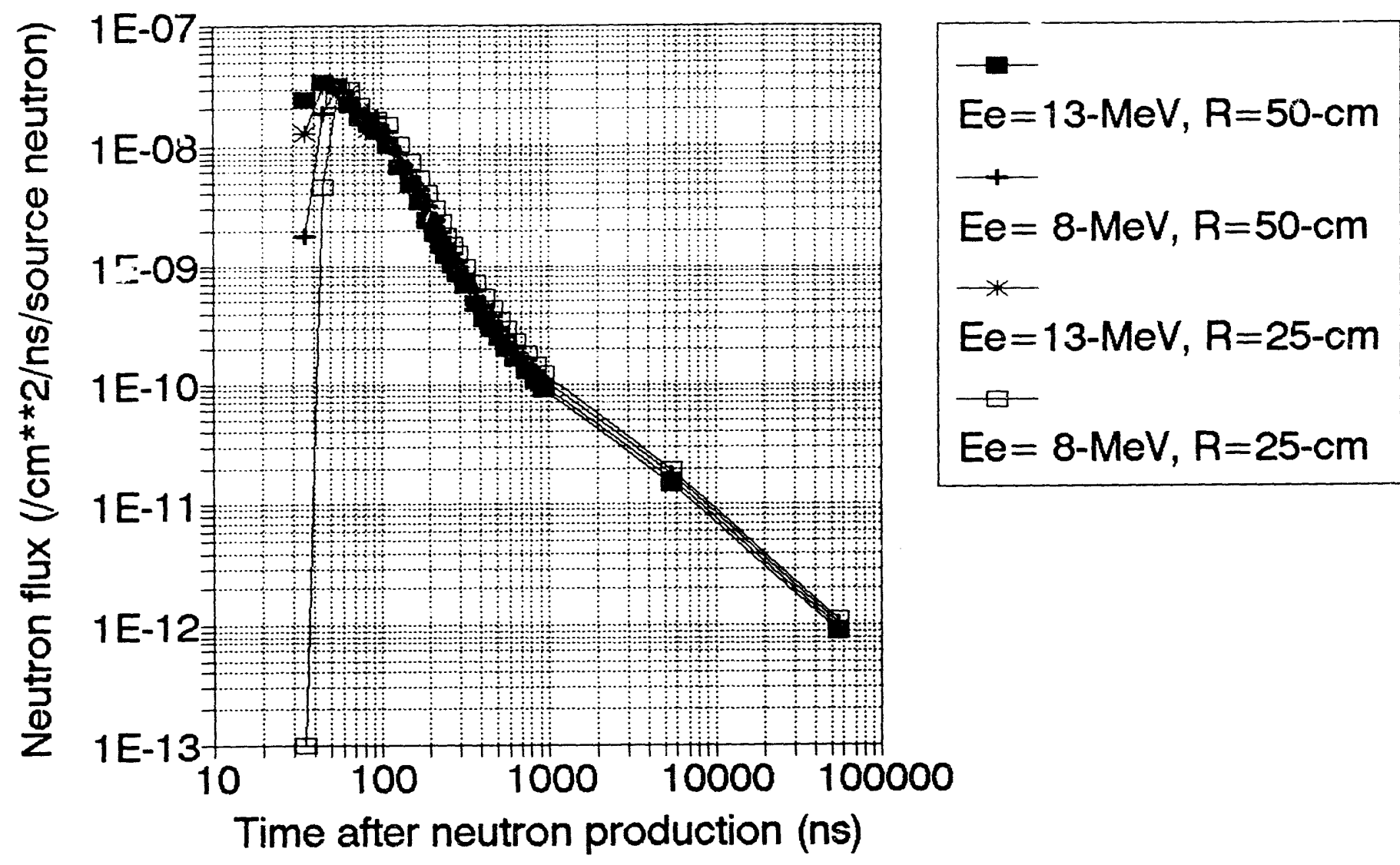

Figure 4.7 Detector location temporal neutron responses for mustard gas with several electron beam energies and object sizes (i.e., Cases 1,4,5, and 8 ). 



Figure 4.8 Detector location temporal gamma responses for various object materials and $13-\mathrm{MeV}$ electrons (i.e., Cases 1-3). 


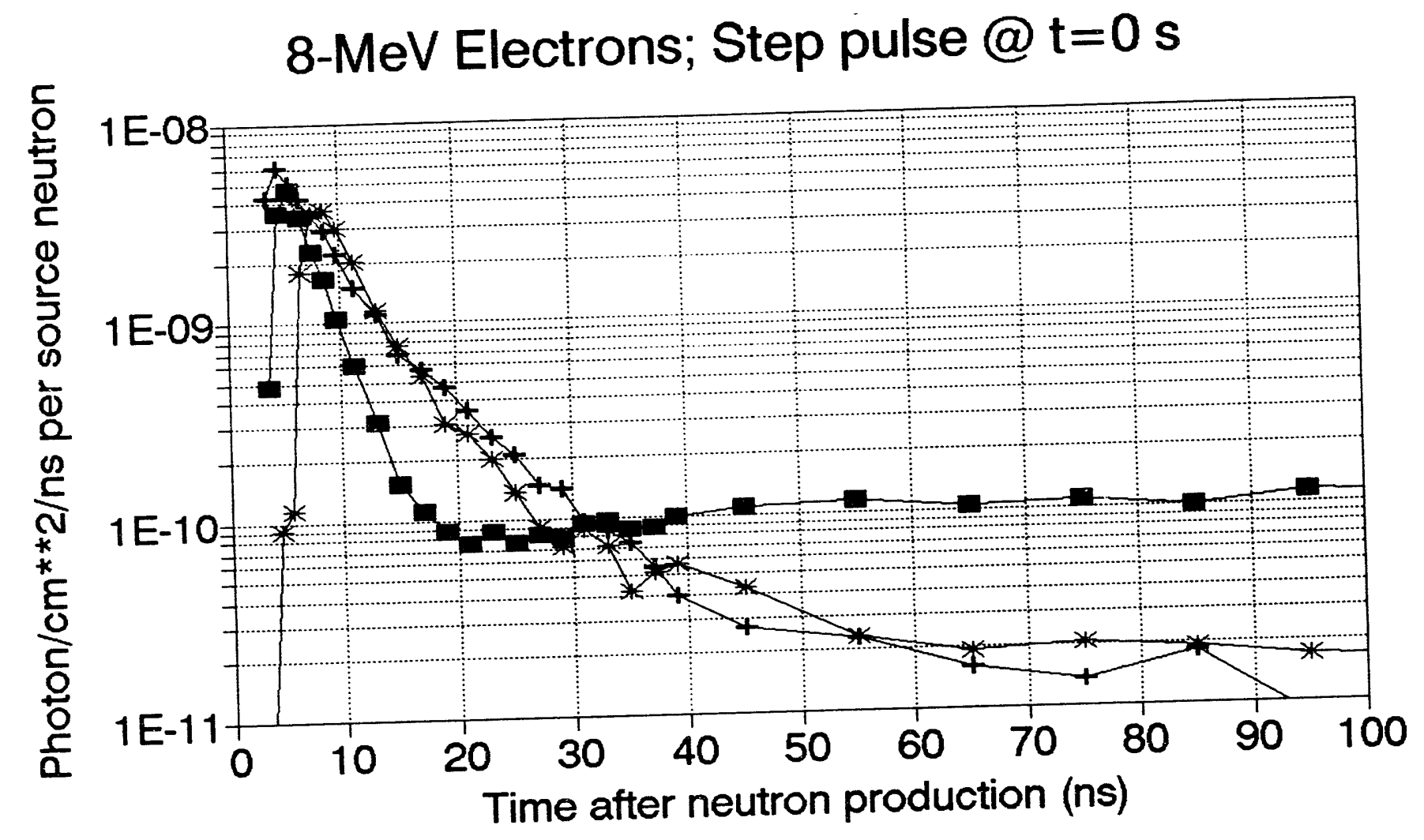

$\rightarrow$ Mustard Gas + Concrete $\rightarrow$ Fe-Poly

Figure 4.9 Detector location temporal gamma responses for various object materials and 8-MeV electrons (i.e., Cases 1-3). 


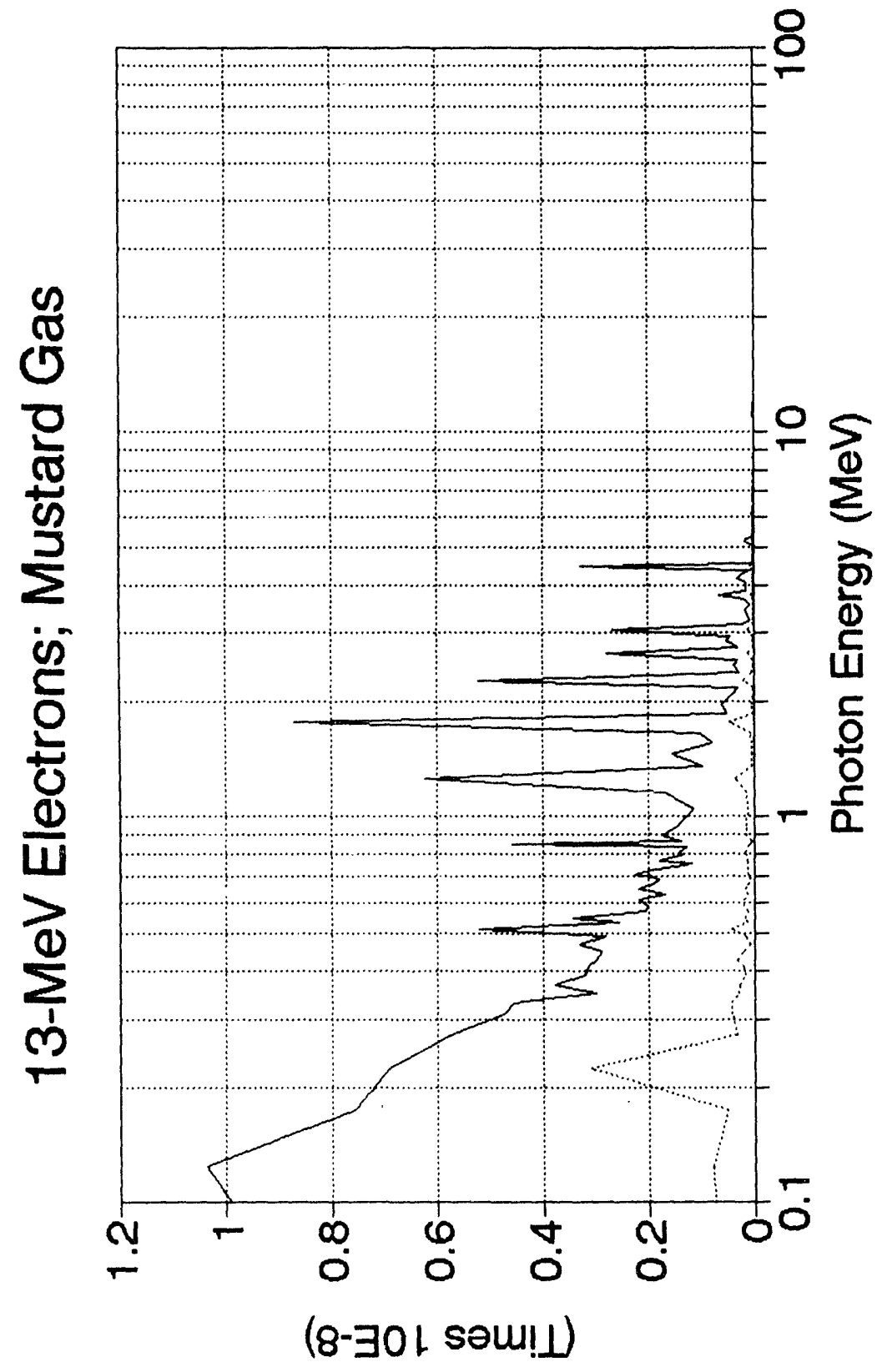

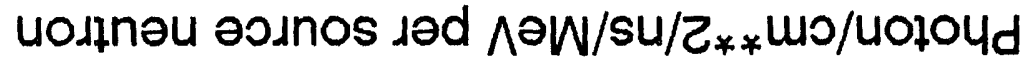

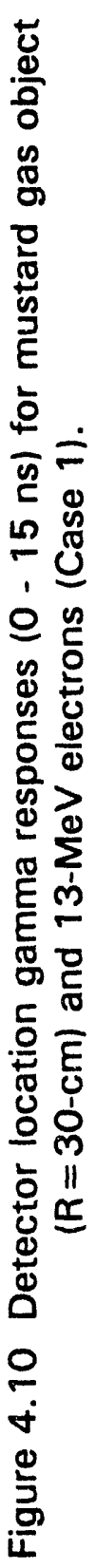




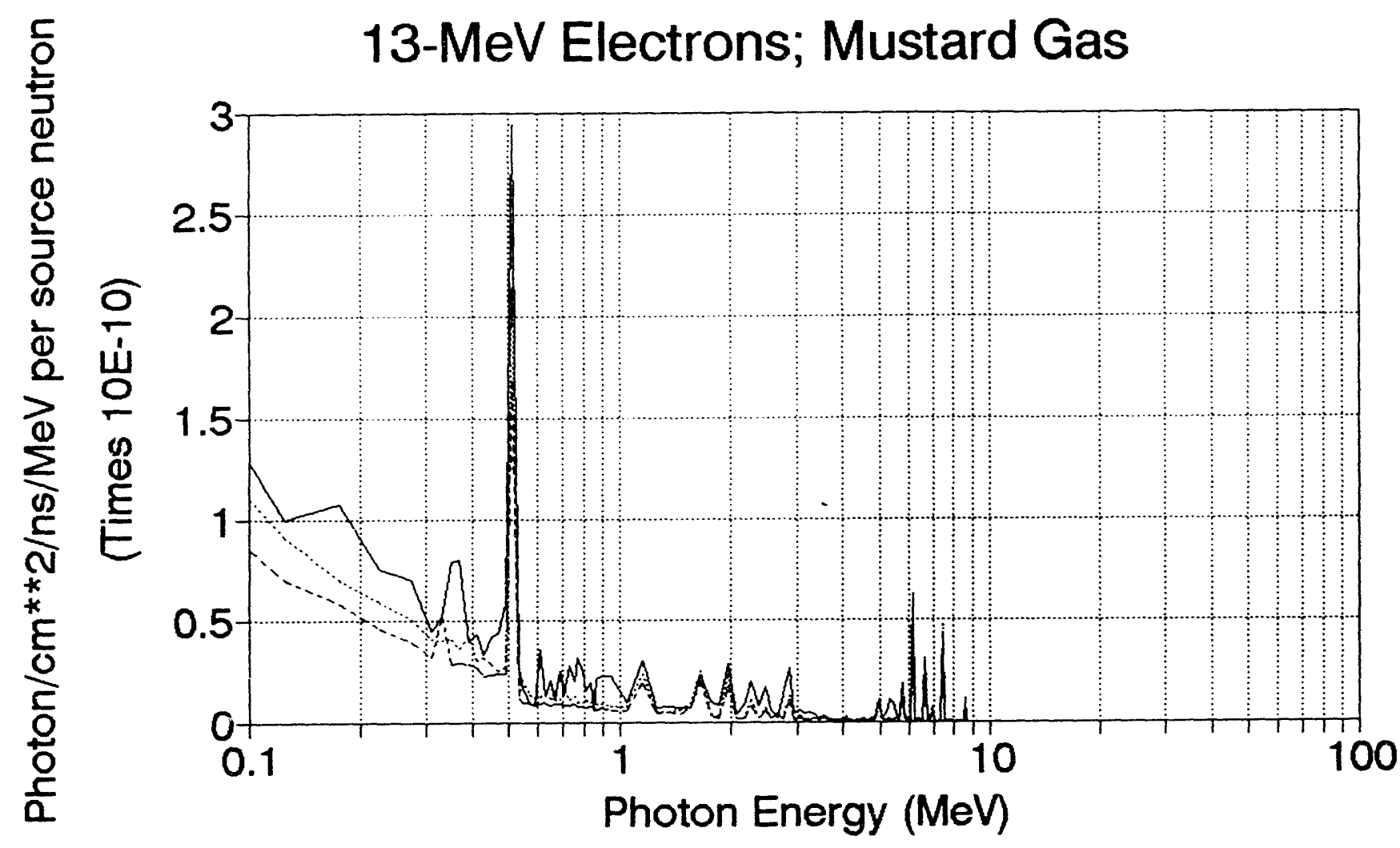

\section{$85-95$ ns \\ $2500-3500$ ns \\ 4500-5500 ns}

Figure 4.11 Detector location gamma responses (80 - $5000 \mathrm{~ns}$ ) for mustard gas object $(R=50-\mathrm{cm})$ and $13-\mathrm{MeV}$ electrons (Case 1 ). 


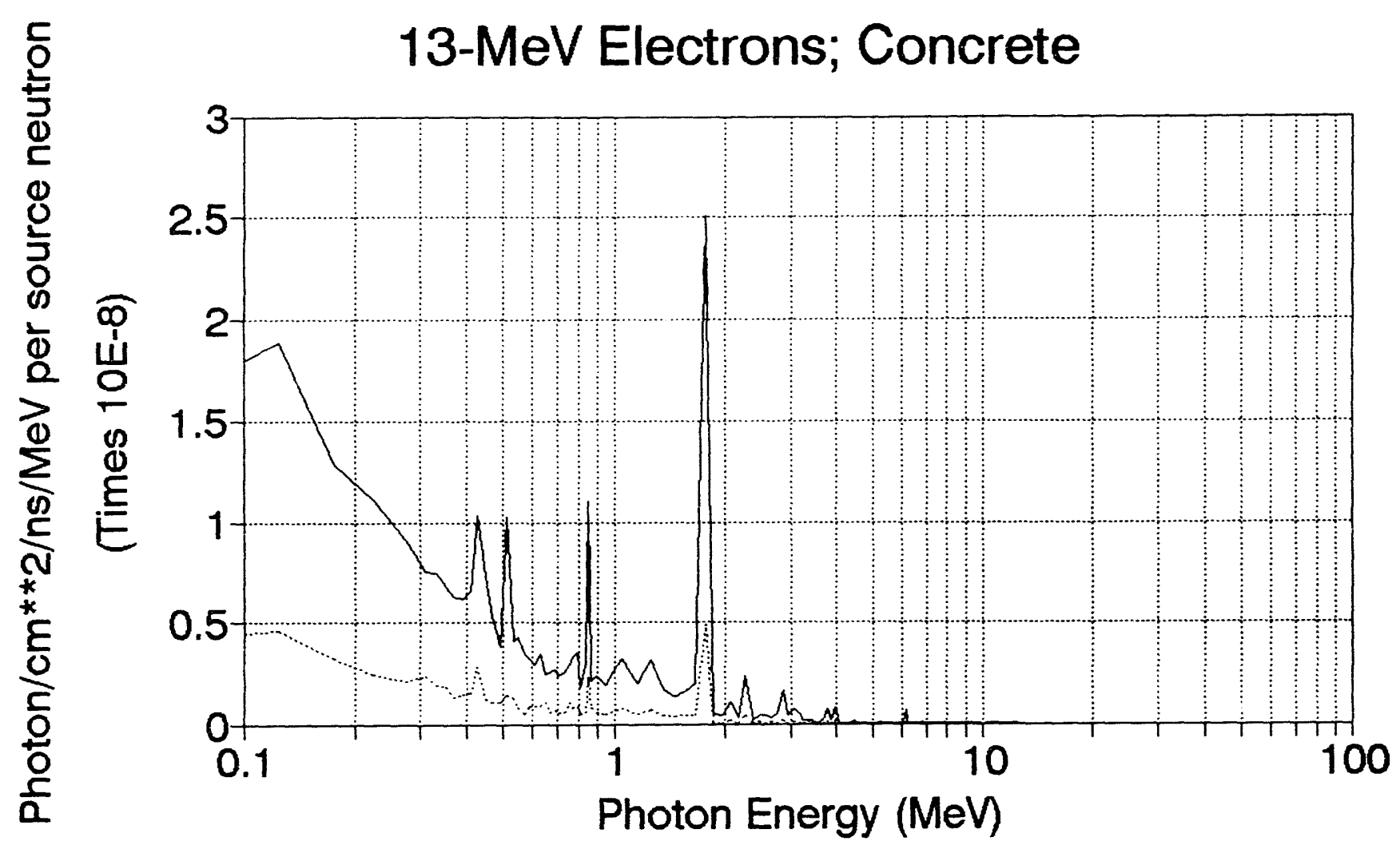

\section{5-7.5 ns $\quad 13-15 \mathrm{~ns}$}

Figure 4.12 Detector location gamma responses ( 0 - $15 \mathrm{~ns})$ for concrete object $(R=50-\mathrm{cm})$ and $13-\mathrm{MeV}$ electrons (Case 2$)$. 


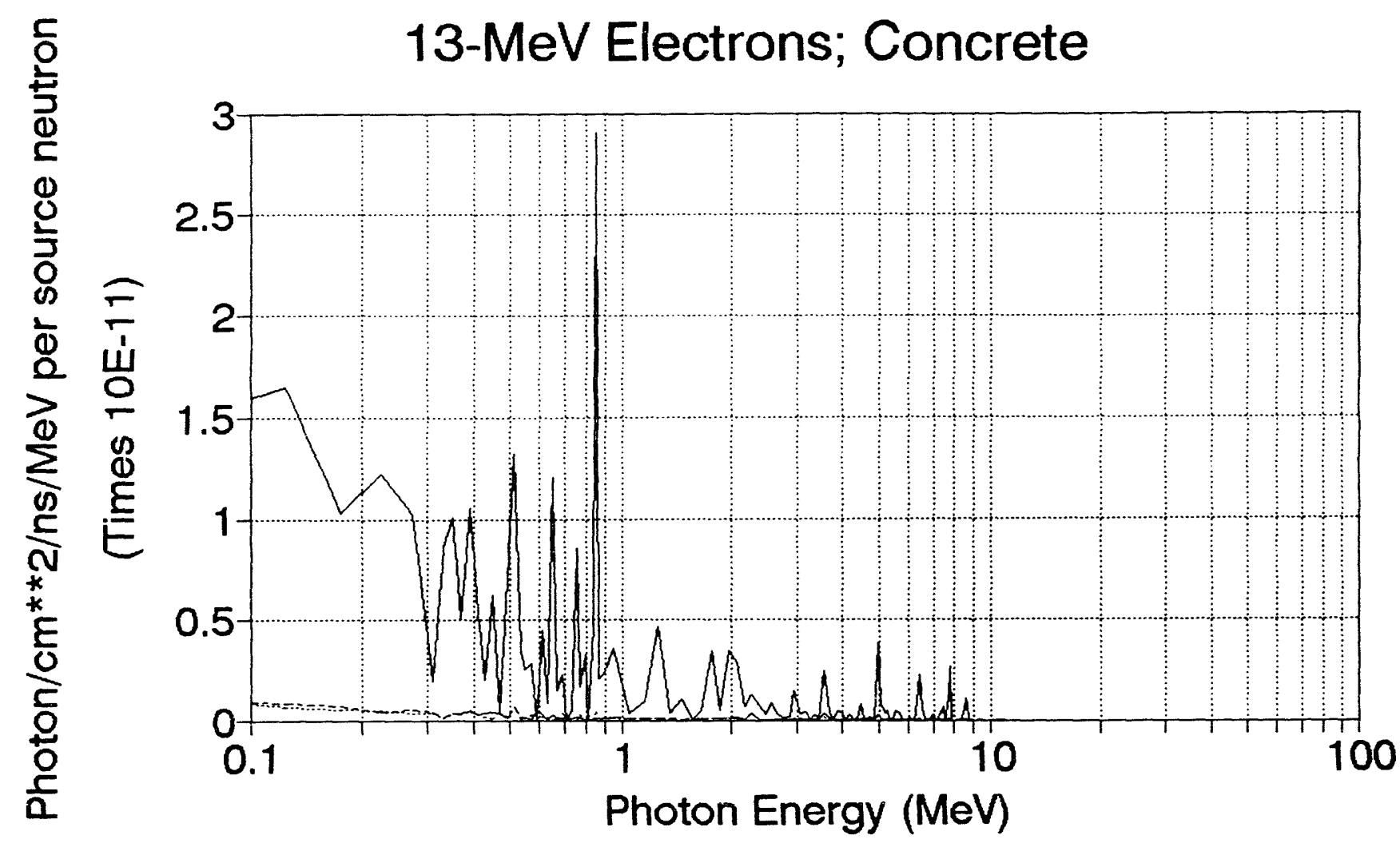

\section{- 85-95 ns $\quad \cdots \cdots \cdots \cdots$}

Figure 4.13 Detector location gamma responses (80-5000 ns) for concrete object $(R=50-\mathrm{cm})$ and $13-\mathrm{MeV}$ electrons (Case 2). 


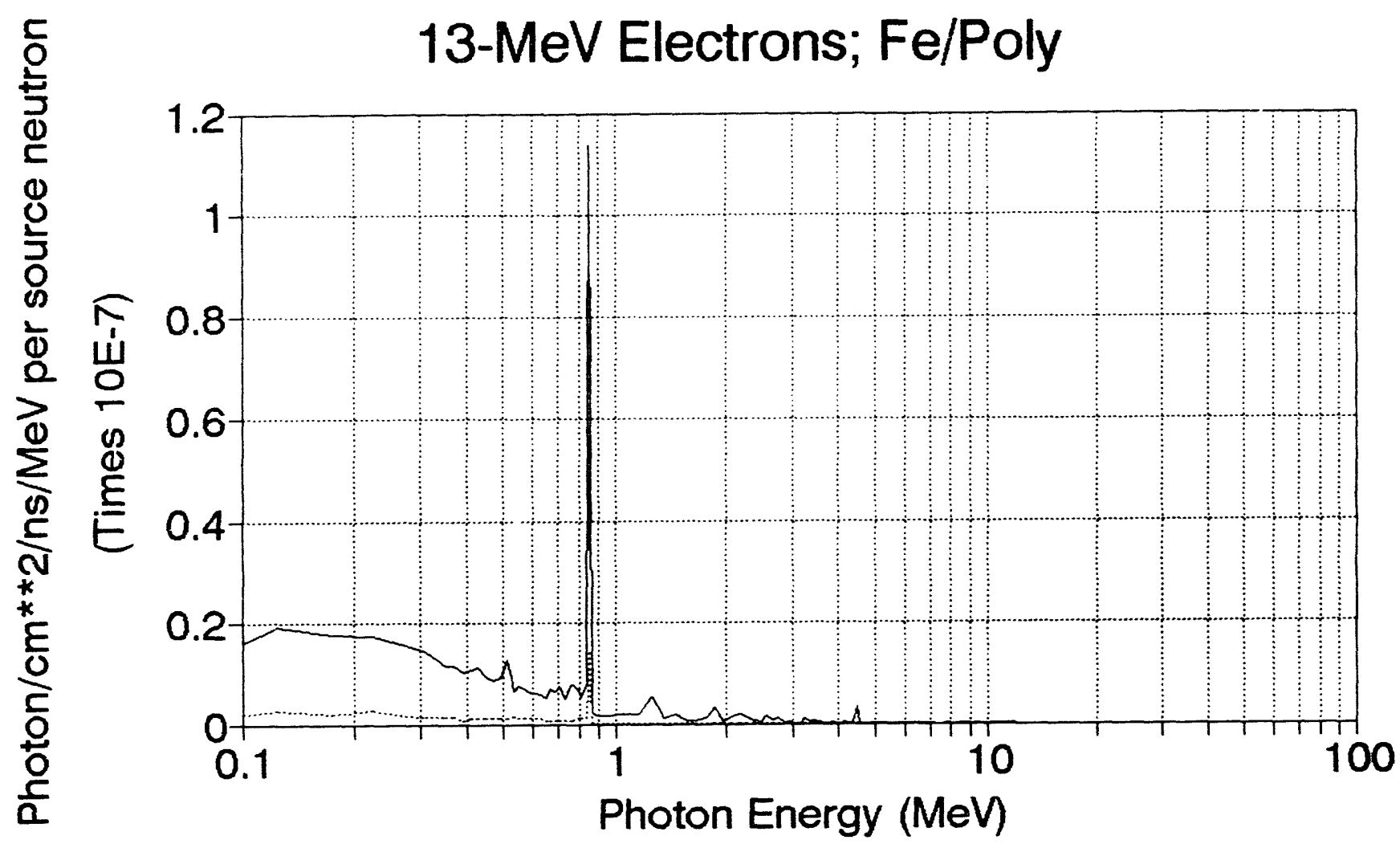

\section{5-7.5 ns ………13-15 ns}

Figure 4.14 Detector location gamma responses (0-15 ns) for Fe/Poly object $(R=45 / 50-\mathrm{cm})$ and $13-\mathrm{MeV}$ electrons (Case 3$)$. 


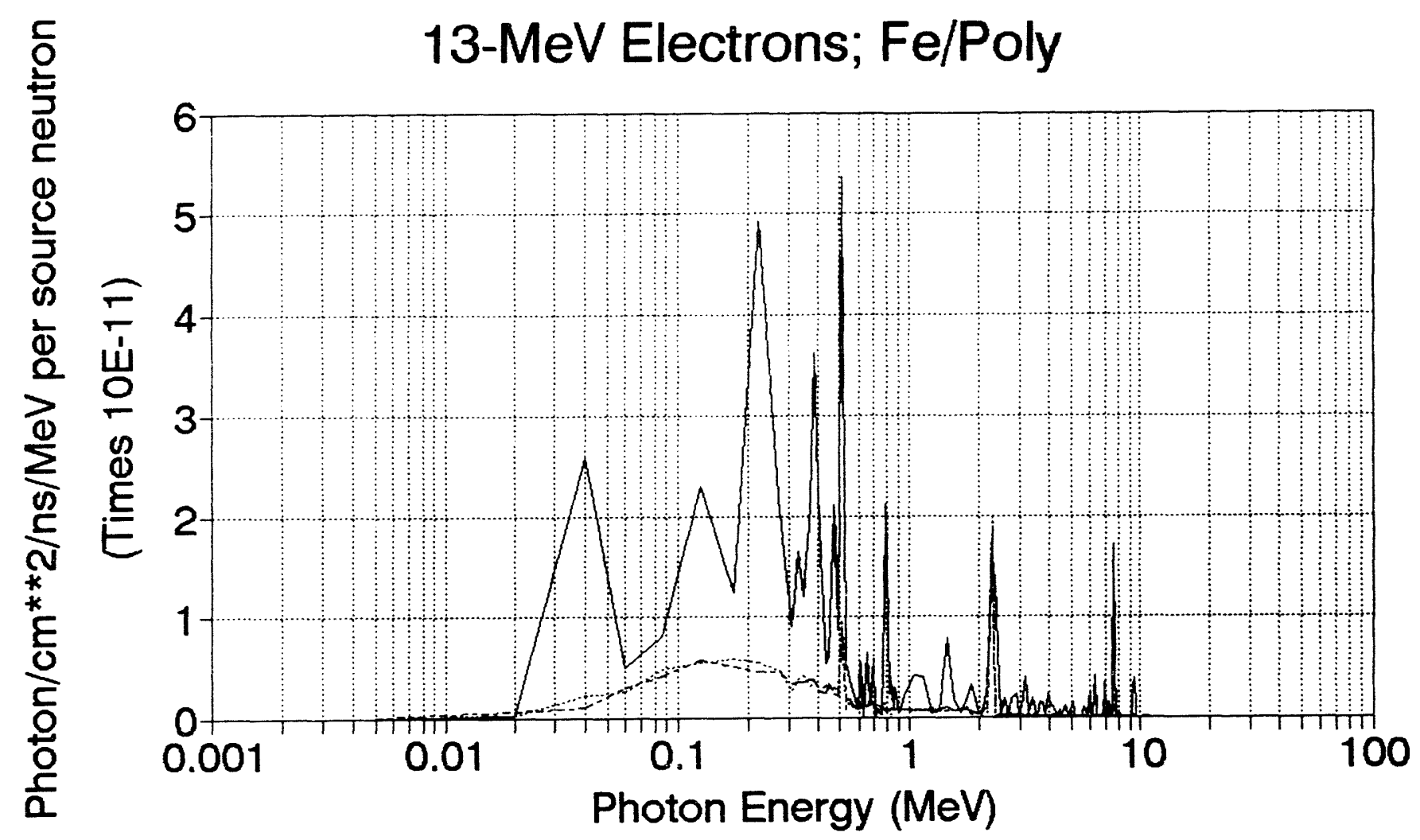

85-95 ns

$2500-3500$ ns

4500-5500 ns

Figure 4.15 Detector location gamma responses (80 - $5000 \mathrm{~ns}$ ) for Fe/Poly object $(R=45 / 50-\mathrm{cm})$ and $13-\mathrm{MeV}$ electrons (Case 3$)$. 


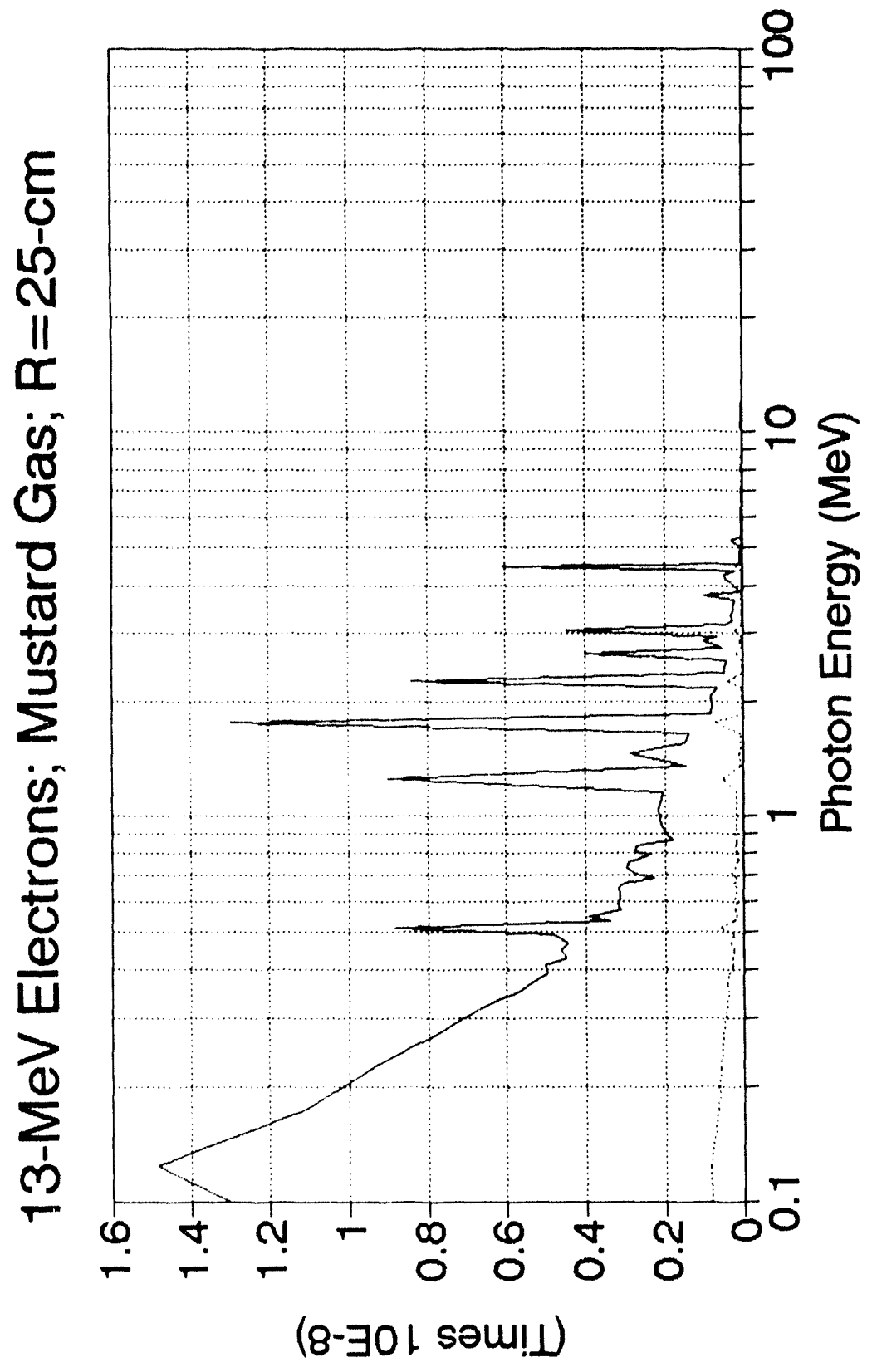

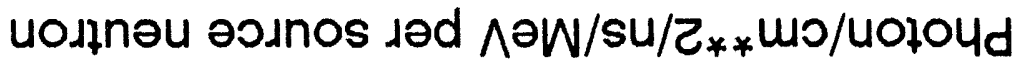

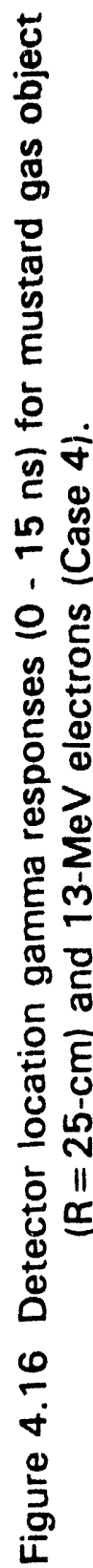






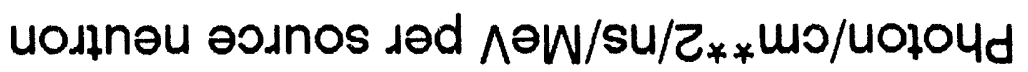

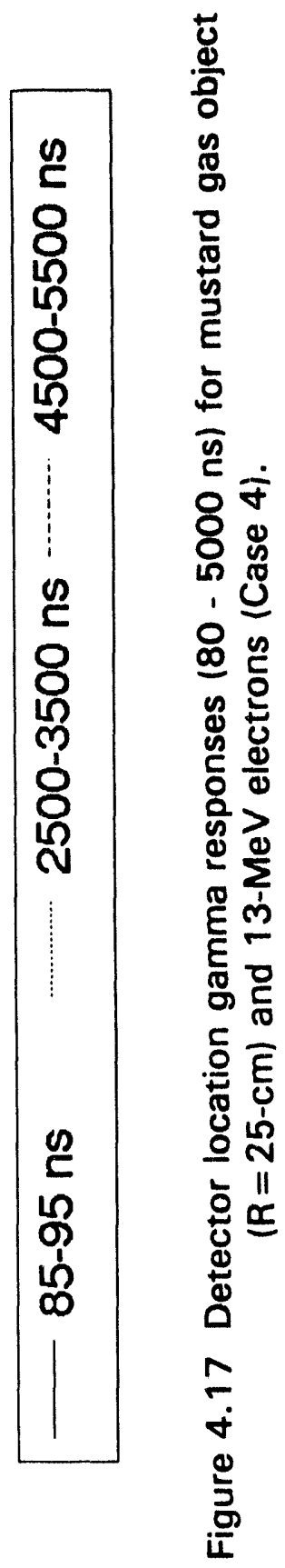




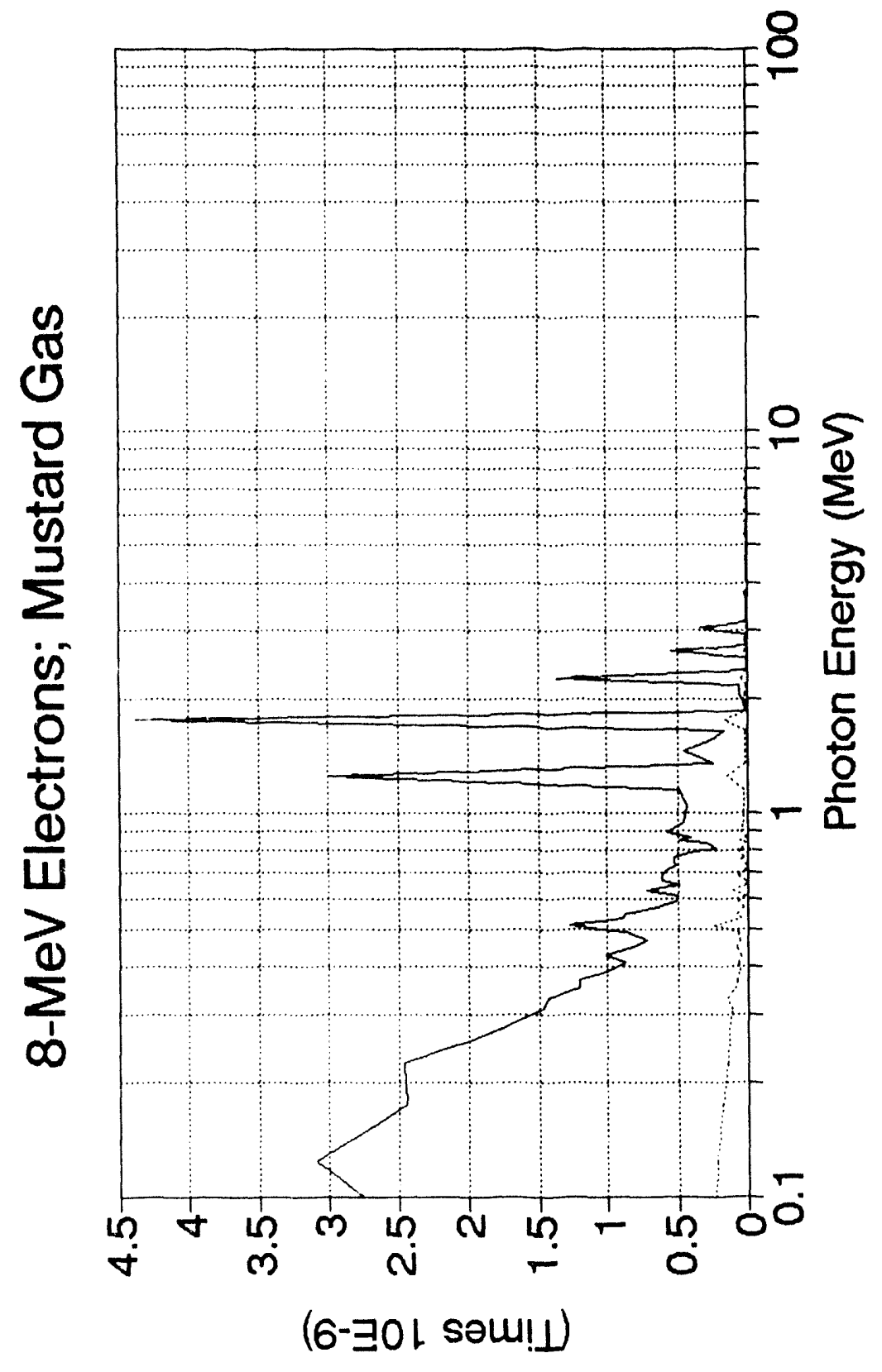

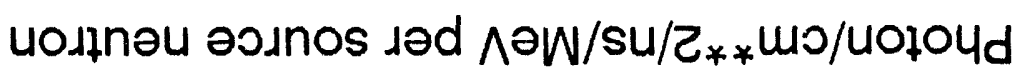

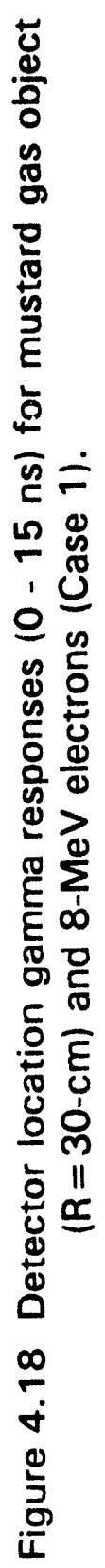






- $85-95 \mathrm{~ns} \quad 2500-3500 \mathrm{~ns} \cdots \cdots \cdots$

Figure 4.19 Detector location gamma responses ( 80 - $5000 \mathrm{~ns}$ ) for mustard gas object $(R=50-\mathrm{cm})$ and 8-MeV electrons (Case 1 ). 


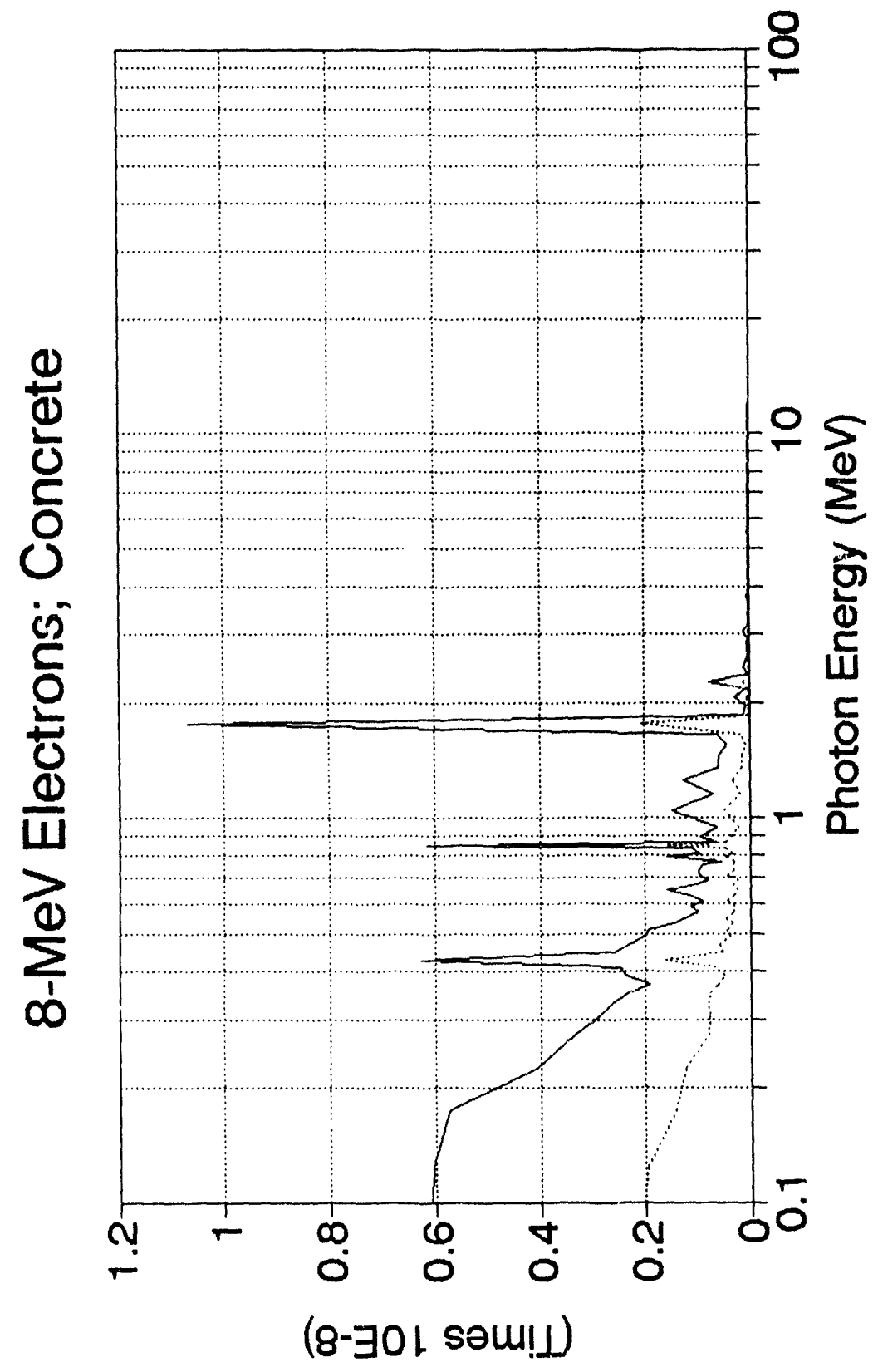

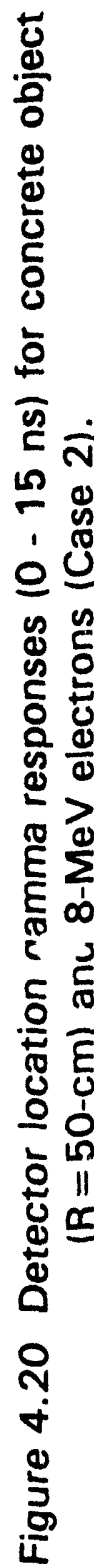







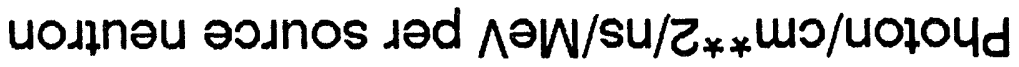


Photon/cm**2/ns/MeV per source neutron

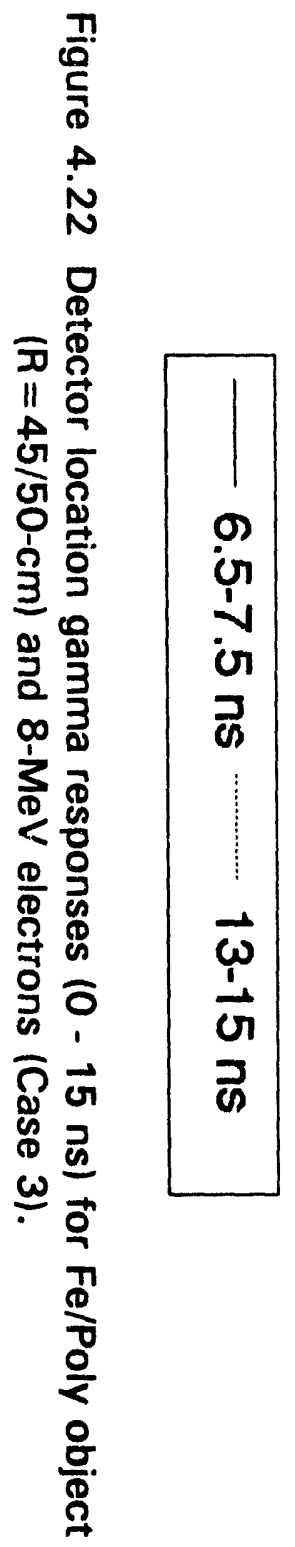

(Times 10E-8)

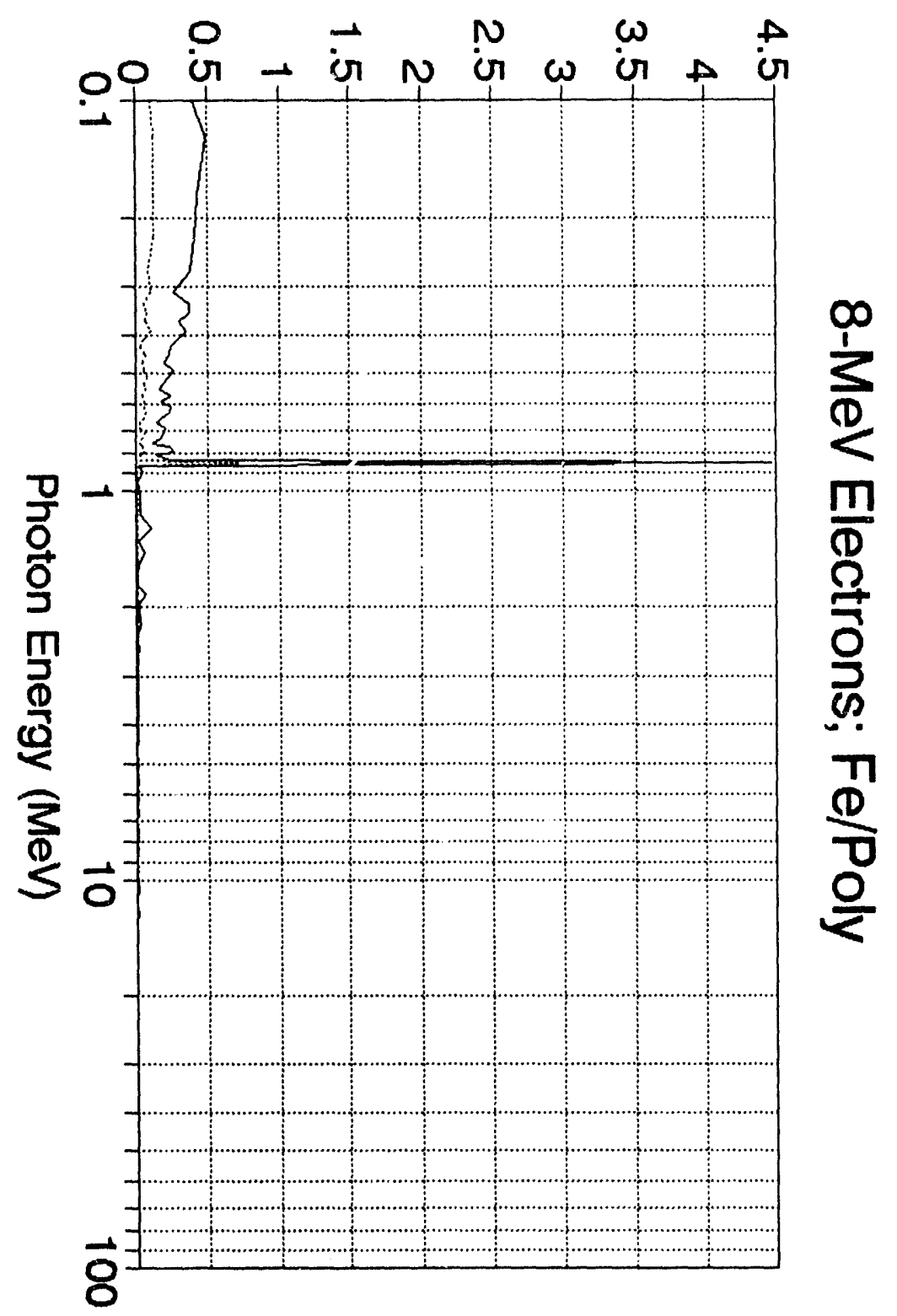




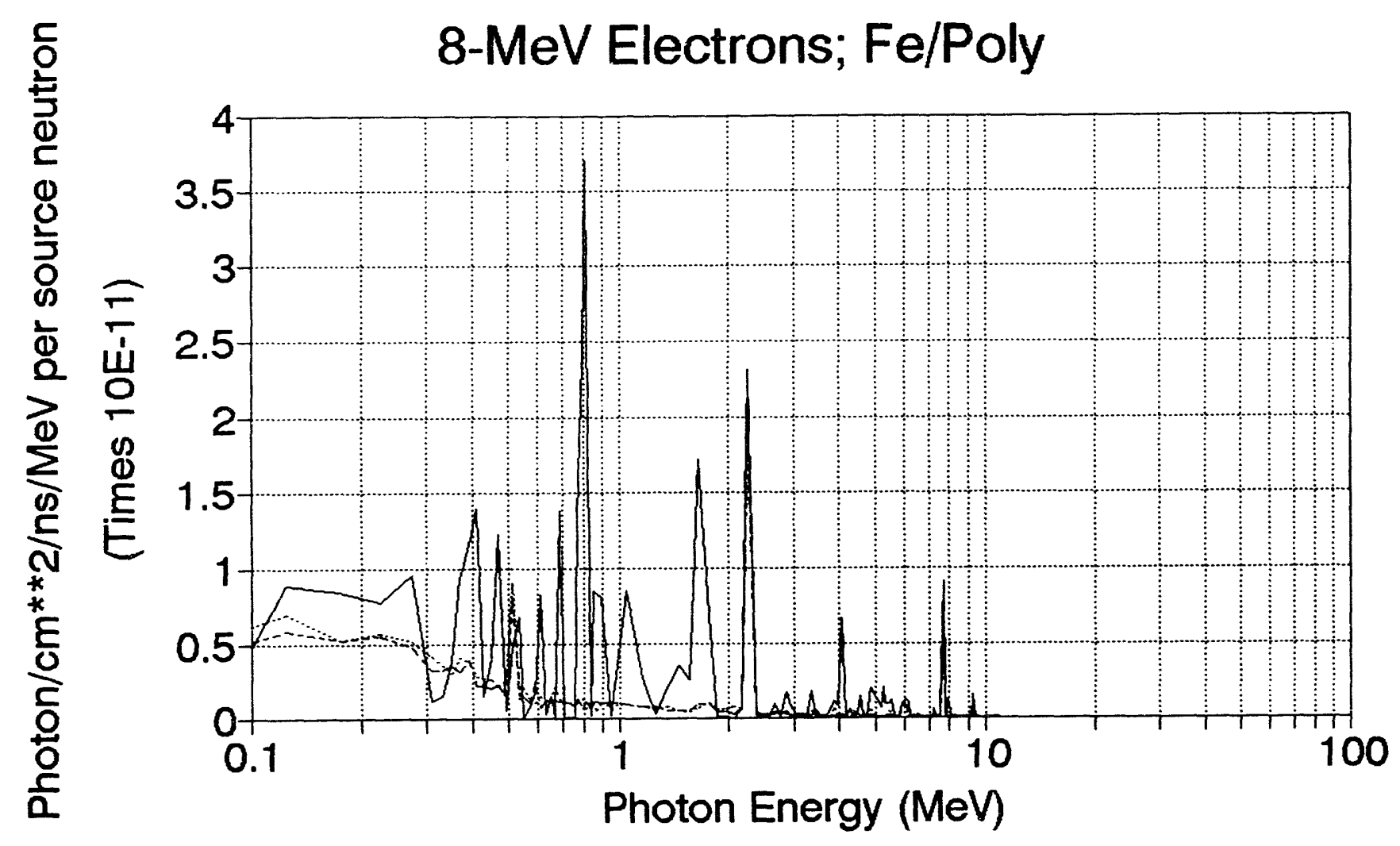

\section{$85-95$ ns \\ $2500-3500$ ns \\ $4500-5500$ ns}

Figure 4.23 Detector location gamma responses ( 80 - $5000 \mathrm{~ns}$ ) for Fe/Poly object $(R=45 / 50-\mathrm{cm})$ and $8-\mathrm{MeV}$ electrons (Case 3$)$. 




Photon/cm ${ }^{\star \star 2} / \mathrm{ns} / \mathrm{MeV}$ per source neutron

(Times 10E-9)

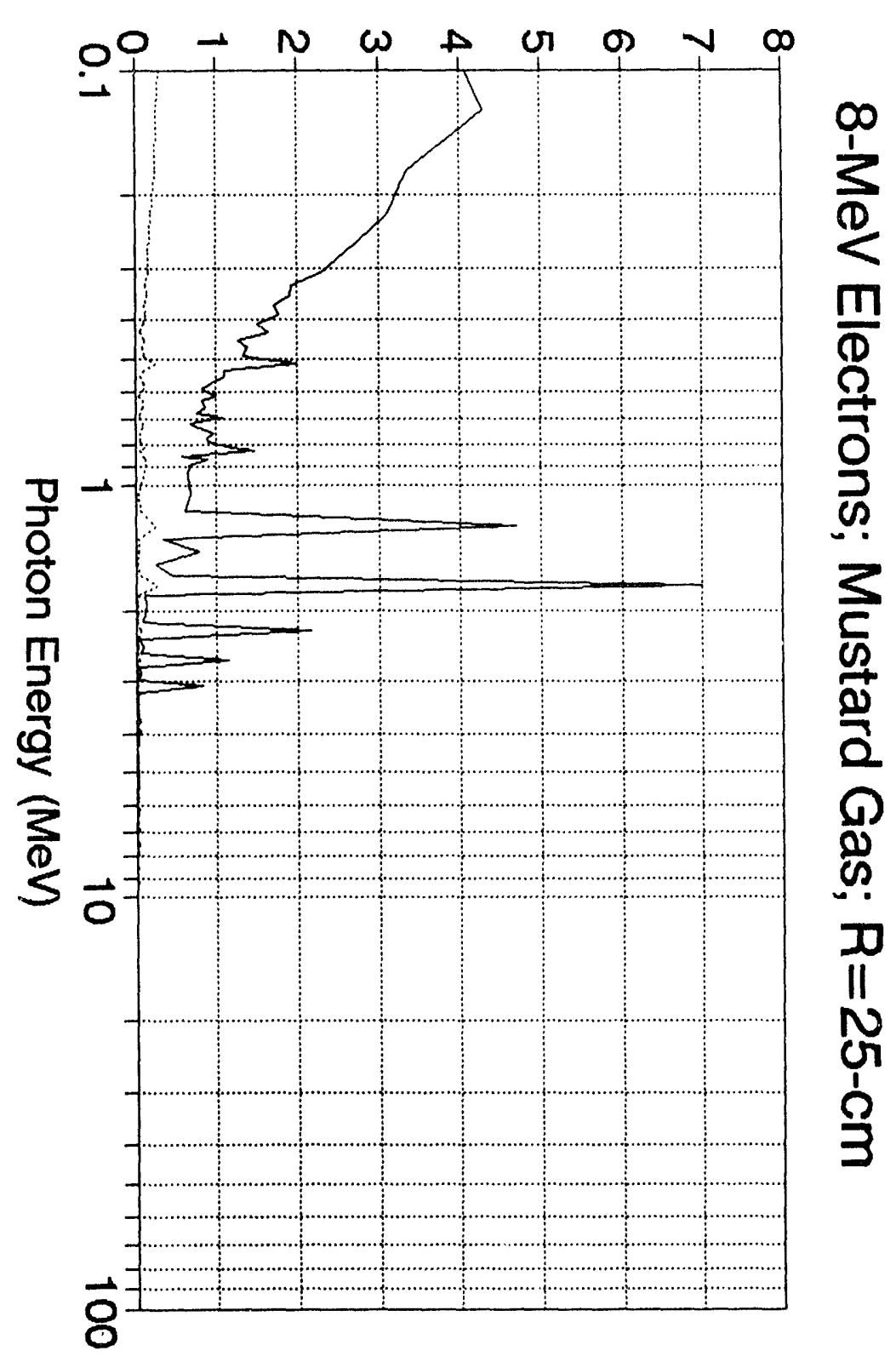




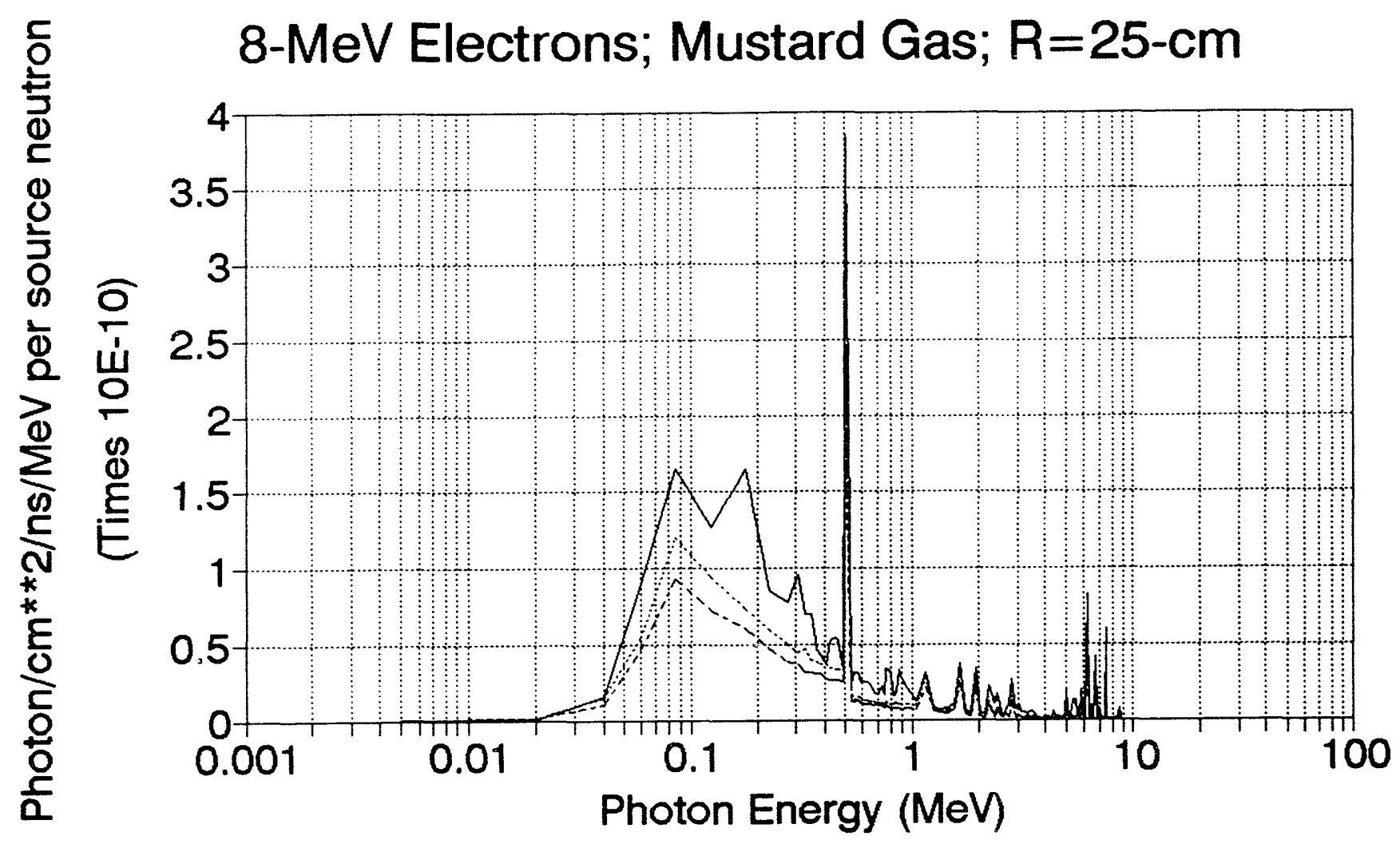

\section{$85-95$ ns $\quad 2500-3500$ ns $\cdots \cdots \cdots$}

Figure 4.25 Detector location gamma responses ( 80 - 5000 ns) for mustard gas object $(R=25-\mathrm{cm})$ and $8-\mathrm{MeV}$ electrons (Case 4). 


\begin{tabular}{|c|c|c|c|c|c|}
\hline & & & & Gamma Respons & tector Location \\
\hline Case & Material & $\begin{array}{c}\text { Electron } \\
\text { Energy } \\
(\mathrm{MeV}) \\
\end{array}$ & $\begin{array}{r}\text { Sphere } \\
\text { Radius } \\
(\mathrm{cm})\end{array}$ & $\begin{array}{l}0-15 \mathrm{~ns} \\
\text { Figure Number }\end{array}$ & $\begin{array}{r}80-5000 \text { ns } \\
\text { Figure Number }\end{array}$ \\
\hline 1 & MG & 13 & 50 & 4.10 & 4.11 \\
\hline 2 & Conc & 13 & 50 & 4.12 & 4.13 \\
\hline 3 & $\mathrm{Fe} / \mathrm{P}$ & 13 & $45 / 50$ & 4.14 & 4.15 \\
\hline 4 & MG & 13 & 25 & 4.16 & 4.17 \\
\hline 5 & MG & 8 & 50 & 4.18 & 4.19 \\
\hline 6 & Conc & 8 & 50 & 4.20 & 4.21 \\
\hline 7 & $\mathrm{Fe} / \mathrm{P}$ & 8 & $45 / 50$ & 4.22 & 4.23 \\
\hline 8 & MG & 8 & 25 & 4.24 & 4.25 \\
\hline $\begin{array}{l}\text { MG - } \\
\text { Conc } \\
\mathrm{Fe} / \mathrm{P} \text {. }\end{array}$ & tard $\mathrm{Ga}$ & ylene & & & \\
\hline
\end{tabular}

In all cases, the initial fast time responses are dominated by inelastic neutron scattering events which are about two to three orders of magnitude larger than for the slower time responses (i.e., see fluxes in Figures 4.10 and 4.11). For the three materials studied, all clearly produce unique gamma signatures. Comparisons of Figures 11 and 17 for $13-\mathrm{MeV}$ operation and Figures 19 and 25 for $8-\mathrm{MeV}$ operation indicate that object size does not adversely affect gamma signatures. 


\title{
5. TEMPORAL DETECTOR RESPONSE ASSESSMENTS
}

In late February 1993, the INEL performed detector response tests ${ }^{18}$ at the EG\&G Energy Measurements Linear Accelerator Facility near Santa Barbara, California to characterize the temporal response of candidate fast scintillation materiais following accelerator-produced bursts of high-intensity, pulsed X-rays.

\subsection{Experiment Description}

For these tests the accelerator was operated in a minimal $\mathrm{X}$-ray production mode (especially for the large electron beam currents used). The LINAC testing conditions are shown in Table 5.1.

\section{TABLE 5.1 LINAC TESTING CONDITIONS}

\author{
Electron Beam Energy: $16 \mathrm{MeV}$ \\ Electron Beam Peak Current: $12 \mathrm{~A}$ to $80 \mathrm{~A}$ \\ Pulse Repetition Rate: Single pulse to $60 \mathrm{~Hz}$ \\ Pulse Width: 50 picoseconds
}

\begin{abstract}
A "plan-view" of the experimental configuration is shown in Figure 5.1. The equipment was set up adjacent to the 90-degree port in the accelerator facility. The detector assembly was horizontally aligned with the beam line, which was positioned 1.5 meters from the floor and 1.8 meters from the ceiling. A shadow shield of approximately $7.5-\mathrm{cm}$ thick lead surrounded the detectors in all but the front wall field-of-view direction and the immediate backward direction. For this fixed configuration, the initial $X$-ray flash (produced at the electron beam window) arrived at the detector assembly within $4 \mathrm{~ns}$, while the room scatter return (primarily from the forward wall) occurred within 20 ns after each X-ray flash.
\end{abstract}

The detector assembly included two detectors, each consisting of a scintillator material optically coupled to a fast Hamumatsu Type-H3378 photomultiplier tube (PMT). One of the PMT's had a quartz window for use with scintillators having short wavelength light emissions, the other had a standard glass (borosilicate) window. The detector with the BC-418 scintillator was mounted on the PMT with the glass window and was used as a reference throughout the tests. The quartz window PMT was used for the other scintillators. Using a Cs-137 source positioned between the two detectors in the assembly, the high voltage applied to a PMT was adjusted to produce a common pulse height of about $20 \mathrm{mV}$ to enable single photon interaction comparisons for each scintillator material. 




Figure 5.1 "Plan view" of experimental configuration. 
The output signal, for the anode of each PMT, was fed over RG58 coaxial cable to the input of a Tektronics 7104 oscilloscope in the control room. The detector response data were recorded as oscilloscope traces on Polaroid photographs. The signal cabling for this experiment utilized the patch panels and internal cabling at the accelerator facility. As a result of this unoptimized cabling, some of the displayed oscilloscope signals were distorted by mismatched cable connections and by signal ringing. These distortions can be minimized or eliminated in the future by using dedicated, impedance-matched, continuous transmission cables.

\subsection{Results}

Figure 5.2 shows oscilloscope traces of the time response including the Compton and photopeak responses of the 2" $\times 2 " \mathrm{Cs}$ crystal to single photon interactions. The difference between the two traces is in the time scaling. The full-width-at-half maximum (FWHM) of the $662 \mathrm{keV}$ photopeak pulse is $14 \mathrm{~ns}$, the full-width-attenth-maximum (FWTM) is $60 \mathrm{~ns}$, and the peak voltage $21 \mathrm{mV}$. Figure 5.3 presents the oscilloscope traces showing the BC-418 time response for the single photon interactions. In this case, the FWHM of the $477 \mathrm{keV}$ Compton edge is about $5 \mathrm{~ns}$, FWTM is $15 \mathrm{~ns}$, and the peak voltage is $16 \mathrm{mV}$. Similar traces were obtained for $1 " \times 1 " \mathrm{Csl}, \mathrm{BaF}_{2}$, and BHTP. The results are given in Table 5.2

TABLE 5.2 SCINTILLATOR TIME RESPONSE PARAMETERS FOR SINGLE PHOTON INTERACTIONS

\begin{tabular}{|c|c|c|c|c|}
\hline Scintillator & Rise Time & FWHM $^{\mathrm{a}}$ & FWTM $^{\mathrm{b}}$ & Peak Voltage \\
\hline $\operatorname{Csl}\left(2^{\prime \prime} \times 2 "\right)$ & $3 \mathrm{~ns}$ & $14 \mathrm{~ns}$ & $60 \mathrm{~ns}$ & $21 \mathrm{mV}$ \\
\hline CsI (1"X1") & $4 \mathrm{~ns}$ & $10 \mathrm{~ns}$ & $60 \mathrm{~ns}$ & $20 \mathrm{mV}$ \\
\hline BC-418(2"x2") & $2 \mathrm{~ns}$ & $5 \mathrm{~ns}$ & $15 \mathrm{~ns}$ & $16 \mathrm{mV}$ \\
\hline BHTP $(2 " \times 2 ")$ & $1 \mathrm{~ns}$ & $4 \mathrm{~ns}$ & $13 \mathrm{~ns}$ & $18 \mathrm{mV}$ \\
\hline $\mathrm{BaF}_{2}\left(2^{\prime \prime} \times 2^{\prime \prime}\right)$ & $2 \mathrm{~ns}$ & $3 \mathrm{~ns}$ & $15 \mathrm{~ns}$ & $16 \mathrm{mV}$ \\
\hline
\end{tabular}



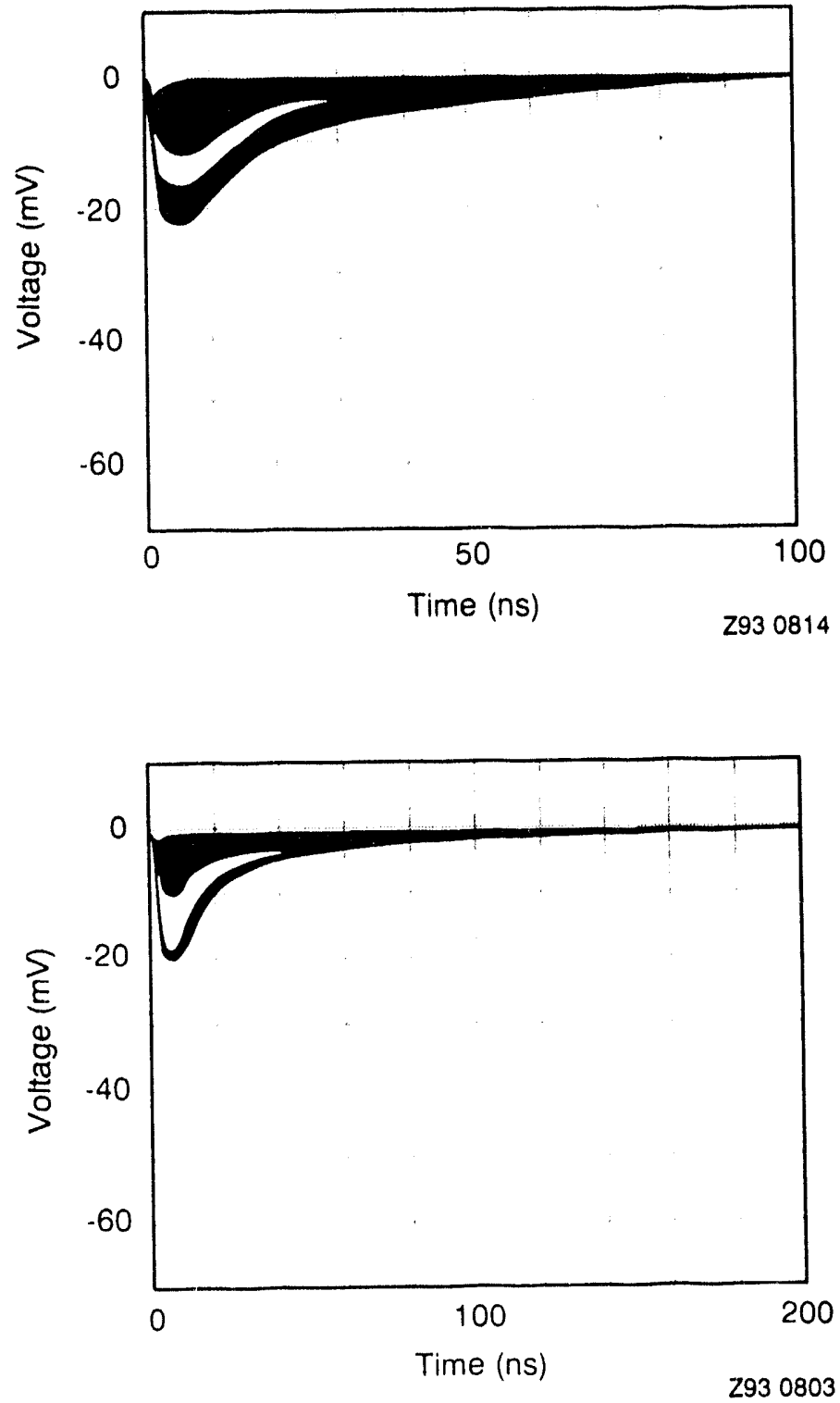

Figure 5.2 "2" 2 2" Csl single photon response. (Note:

Time scale changes). 

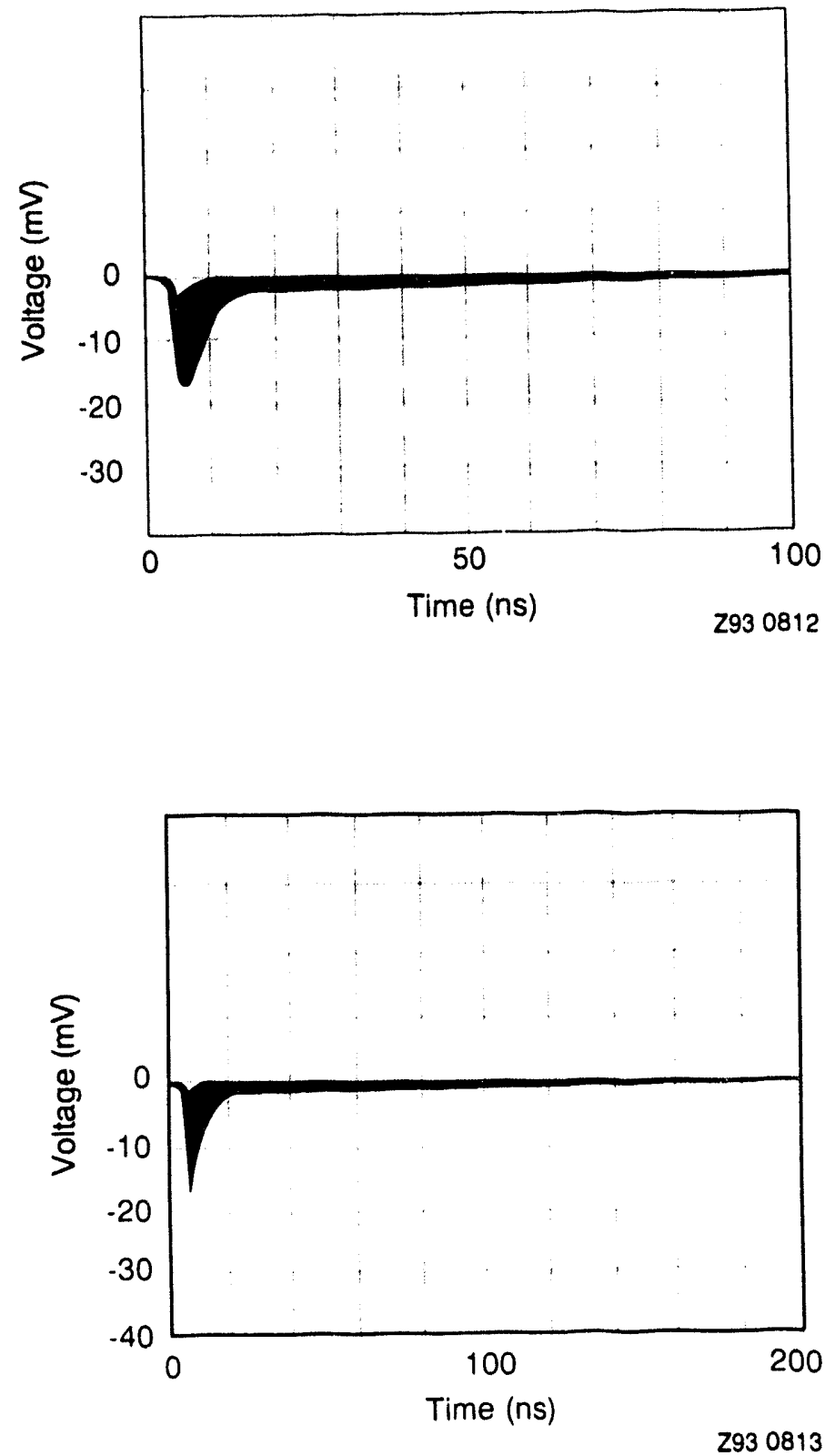

Figure $5.32 " \times 2 " B C-418$ single photon response. (Note: Time scale changes.) 
Figure 5.4 is the oscilloscope trace of the time response of $\mathrm{Csl}(2 " \times 2 ")$ due to multiple photons interacting in the crystal from the $X$-ray flash of the LINAC operating with a 12-A peak electron current. In this particular case, the initial pulse (extended maximum voltage of the scope trace) is the summation of approximately 700 individual photon responses in the crystal during the flash event. The number of photon interactions is dependent on several factors, including the efficiency of the crystal to detect photons, shielding around the detector, intensity of the electron beam, and the energy spectrum of the photons in the $X$-ray flash. Figure 5.4 also shows the electronic ringing (second and third peaks at approximately $360 \mathrm{~ns}$ and $520 \mathrm{~ns}$, respectively, after the start of the initial $X$-ray flash peak) due to the signal cable mis-match.

Figures 5.5 and 5.6 are similar oscilloscope traces at $12-A$ for the $B C-418$ and BHTP, respectively. Note the change in time scales between these two figures. Figure 5.5 shows two different BC-418 traces. Figure 5.6 shows single photon events riding on the tail of the $X$-ray flash for the BHTP. In the upper trace, single photon pulses are observed at $65,125,165$, and 175 ns after the start of the burst. In the lower trace of Figure 5.6, single photon pulses are observed at 75 and $90 \mathrm{~ns}$ after the start of the burst. Identification as single photon pulse was based on the relative pulse shape (some heights as large as $200 \mathrm{mV}$; corresponding to about $7 \mathrm{MeV}$ ) and their random occurrence in time. Figure 5.7 shows the relative comparison between the BC-418 and BHTP responses. In total, 67 polaroid pictures were taken. All are included in the project file for further reference.

Table 5.3 summarizes the time response parameters of the five scintillators for electron LINAC $X$-ray flash interactions. This table also presents the time response parameters for $X$-ray flash effect on the photomultiplier tube without a scintillator attached. (The latter compared well with the manufacturer supplied performance data.)

\subsection{Discussion of Results}

It is evident from a comparison of Tables 5.2 and 5.3 that the pulses are much broader for the $X$-ray flash events than for the single photon events. Much of this broadening is due to the actual time spread of the arrival of the flash at the detector. Each pulse produced a precursor bremsstrahlung source because prior to the electron beam reaching the outlet port, the beam underwent a $90^{\circ}$ bend. This and the scattering of the photons off the walls, floor, and ceiling spread the time of the arrival of the $X$-ray flash over tens of nanoseconds. The resulting data illustrate the challenges of operating these detectors in the real environment of the application. 




Figure $5.42 " \times 2 "$ Csl X-ray flash response for 12 ampere operation. 

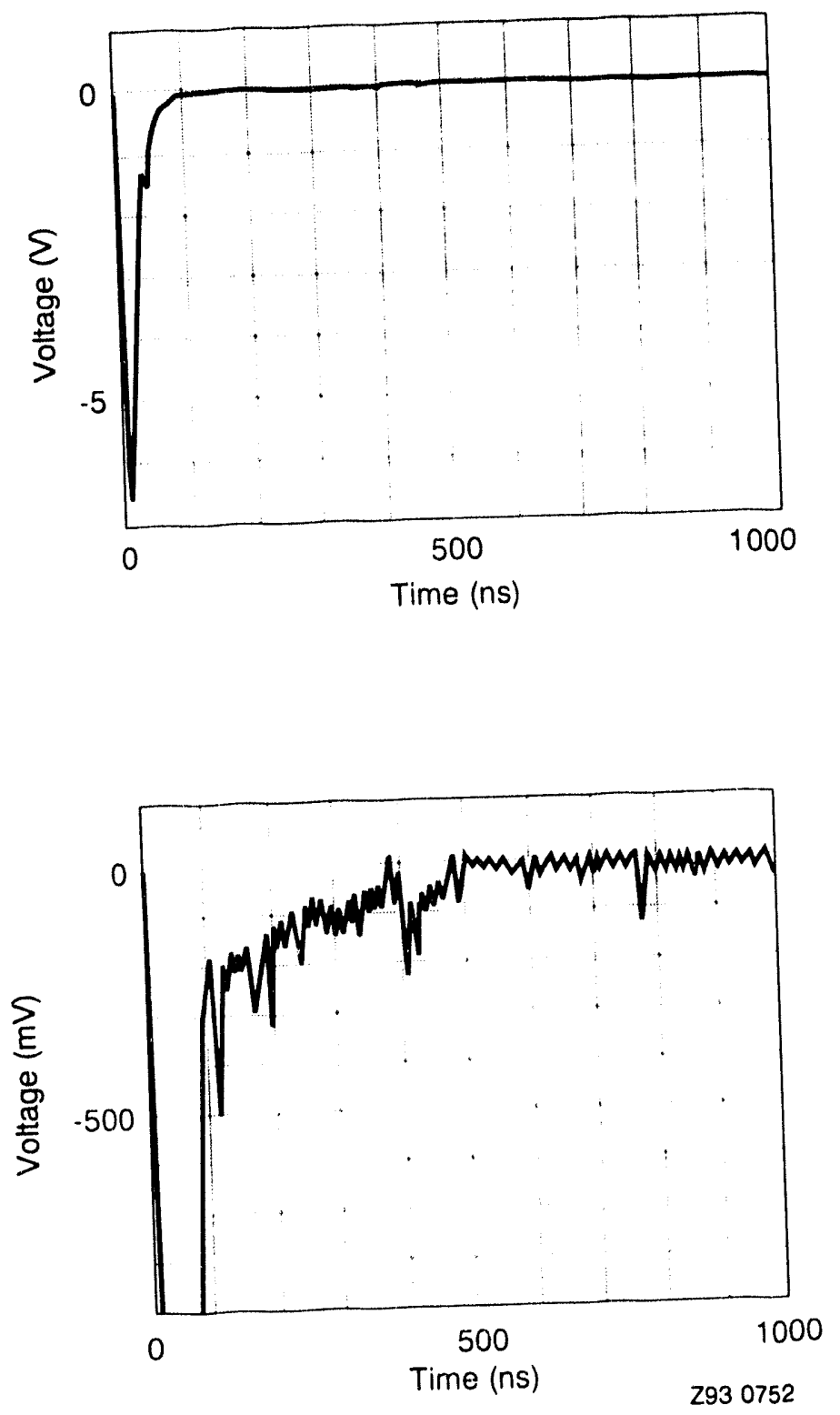

Figure $5.52 " \times 2 " B C-418 \mathrm{X}$-ray flash response for 12 ampere operation. 

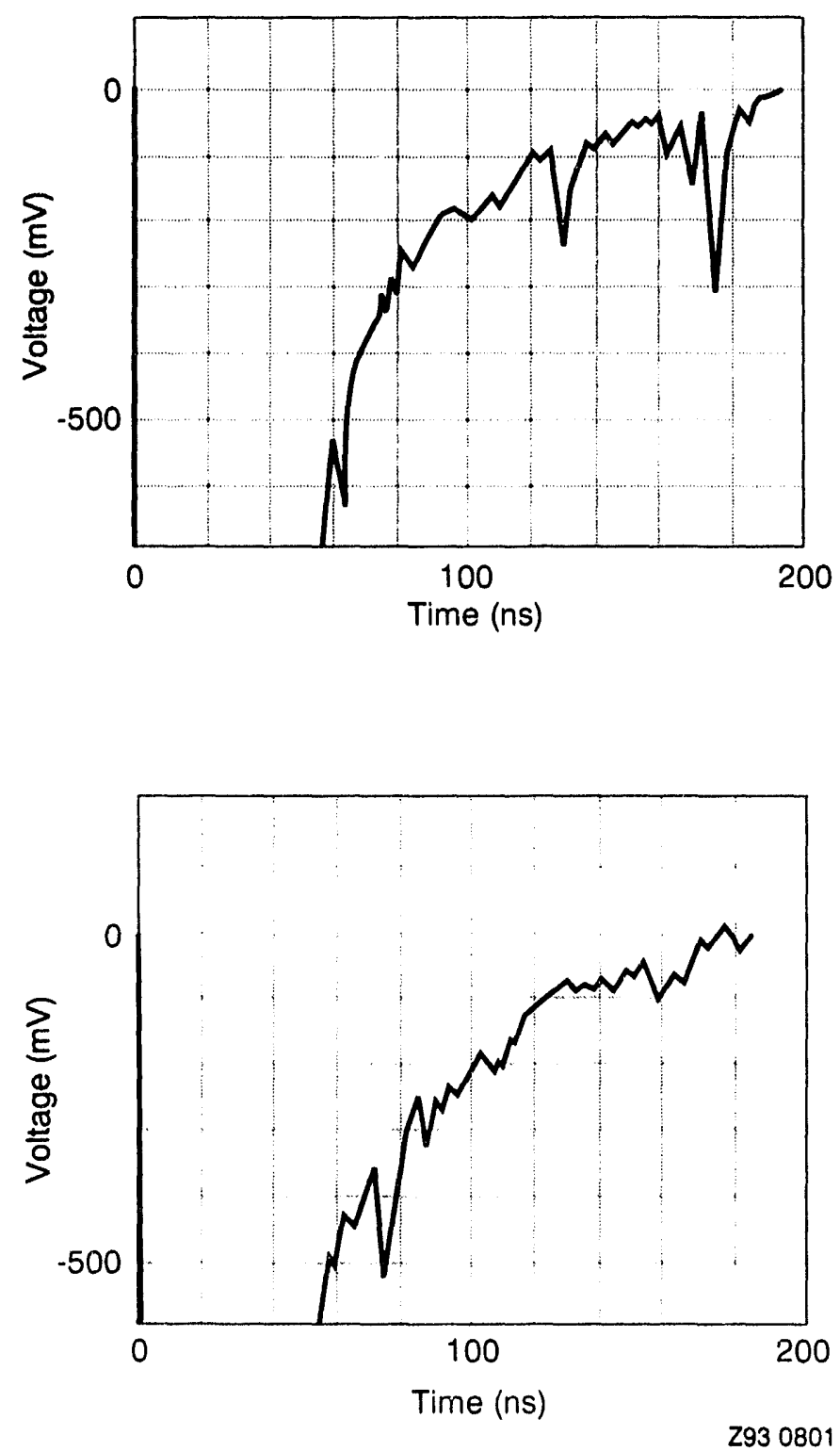

Figure $5.62 " \times 2 "$ BHTP X-ray flash response for 12 ampere operation. (Two separate accelerator pulsed responses) 




Figure 5.7 BC-418 and BHTP relative $X$-ray flash response comparisons. (BHTP has faster recovery time.) 
TABLE 5.3 SCINTILLATOR TIME RESPONSE PARAMETERS FOR ELECTRON LINAC X-RAY FLASH

\begin{tabular}{|c|c|c|c|c|}
\hline Scintillator & FWHM & FWTM & $\begin{array}{l}\text { Peak } \\
\text { Voltage }\end{array}$ & $\begin{array}{r}\text { Electron Beam } \\
\text { Peak Current }\end{array}$ \\
\hline CsI (2"x2") & $130 \mathrm{~ns}$ & $220 \mathrm{~ns}$ & $14 \mathrm{~V}$ & $12 \mathrm{~A}$ \\
\hline CsI (1"x1") & 140 ns & $300 \mathrm{~ns}$ & $17 \mathrm{~V}$ & $40 \mathrm{~A}$ \\
\hline BC-418 (2"x2") & $44 \mathrm{~ns}$ & $80 \mathrm{~ns}$ & $7 v$ & $12 \mathrm{~A}$ \\
\hline BHTP $\left(2 " \times 2^{\prime \prime}\right)$ & $24 \mathrm{~ns}$ & $50 \mathrm{~ns}$ & $11 \mathrm{~V}$ & $12 \mathrm{~A}$ \\
\hline $\mathrm{BaF}_{2}\left(2^{\prime \prime} \times 2 "\right)$ & $1300 \mathrm{~ns}$ & 3800 ns & $14 \mathrm{~V}$ & $40 \mathrm{~A}$ \\
\hline PMT w/o scint. & $8 \mathrm{~ns}$ & $15 \mathrm{~ns}$ & $3 V$ & $25 \mathrm{~A}$ \\
\hline
\end{tabular}

The spread in the X-ray flash can be reduced by shielding the detectors and by improving the geometric configuration. Experience from previous experiments indicates that by using the $0^{\circ}$ port (no beam bending) and using improved detector shielding, the time spread in the X-ray flash can be reduced to less than $10 \mathrm{~ns}$. The greater the beam intensity, the larger the initial flash and the longer it takes for the detectors to recover. Therefore, it is important that the accelerator beam intensity be adjusted so that the detectors recover as soon as possible, and yet, still provide significant measurable single photon signals.

By comparing the time response of different scintillator (oscilloscope traces), single photon pulses were identified on the tail of the X-ray flash pulse as early as 60 ns after the start of the burst. Observing single photon pulses at earlier times was not possible because of the rapid fall off the X-ray flash pulse, combined with the width of the scope trace, obscured the single photon peaks. Single photon events after $60 \mathrm{~ns}$ were observed (on the scope) at specific rates of up to $40 \mathrm{MHz}$. A baseline restorer circuit should be able to remove the $X$-ray flash tail and thereby enable the detection and pulse height analysis of single photon pulses within at least 50 ns after the burst.

BC-418 and BHTP have the best potential for being able to produce detectible 
single photon pulses within 50-60 ns of the burst. Depending on how successful a baseline restorer circuit is in suppressing the tail of the $X$-ray flash, the pure Csl (with its inherent photoelectric gamma response for energy resolution) looks very promising for this application. The slow scintillation component of the $\mathrm{BaF}_{2}$ appears to dominate its response, and therefore, this scintillator material is not suitable for this application.

Based on the time response characteristics and the testing conditions, the organic compound BHTP in polystyrene ranks highest as the detector material for the MIT program. The second best is the plastic BC-418 which is commercially available. Both the BHTP and the $\mathrm{BC}-418$ will require Compton-edge analysis to arrive at a gamma spectrum for identifying materials, and the energy resolution of the resulting gamma spectra are yet to be determined.

Therefore, of the detectors assessed for the MIT technology's fast timing requirements, the $\mathrm{BaF}_{2}$ recovery response is simply too slow. While BHTP is faster than $\mathrm{BC}-418, \mathrm{BC}-418$ has a better Compton energy resolution as seen in Table 3.3. Hence, it appears that faster detection response occurs at the expense of gamma energy resolution. For a final detection system design, it is apparent that some type of compromise will have to be made between these critical detector characteristics. While the time response of the pure Csl is not as fast as the organics, the CsI detector is believed to be the best detector candidate (of the those tested) if a baseline restorer can effectively suppress the tail of the X-ray flash or if the electron beam current can be sufficiently reduced to minimize the flash effect. The Csl has a strong photoelectric gamma response; and standard photopeak spectrometry is possible, which is more straight forward than Comptonedge analysis. 


\section{POTENTIAL DETECTION SYSTEM CONCEPT}

The two basic requirements for a detection system which would accumulate pulse height data in the time between successive accelerator bursts are listed below.

1. The detection system must have a fast response so that it can recover from the accelerator gamma flash and then be able to acquire pulse height data at rates up to $40 \mathrm{MHz}$ in the time region from $30 \mathrm{~ns}$ to $5 \mu$ s following a burst.

2. It must be able to isolate different time regions following each accelerator burst based on: (a) the type of events producing the gamma rays (i.e., neutron inelastic scattering or neutron capture), and (b) the time response limitations of the detector(s), photomultiplier tube(s), and front end electronics.

One detection channel of a potential, low cost, detection system is shown in Figure 6.1. It is expected that there will be multiple detection channels, where each channel is supported by its own pre-amplifier, peak detection circuit followed by a dedicated flash analog-to-digital convertor (ADC). The detection channel would be program gated to acquire data in a specified time region following the accelerator burst. For each detected event, the digital address of the pulse height generated by the flash ADC along with a digital address for the detection channel generated by the logic control module would be transferred to a fast buffer memory. The buffer memory should be sized to support all the data channels in the detection system. The signal processing and data transfer to the buffer memory should be capable of a $40 \mathrm{MHz}$ data rate. At the completion of the data acquisition and transfer to the buffer memory, the buffer memory contents would be transferred through an Acquisition Interface Module (AIM) to the multichannel analyzer (MCA). The transfer rate from the buffer memory to the MCA is much slower, the order of a few $\mathrm{MHz}$, but is expected to be capable of unloading the entire buffer memory contents to the MCA before the next accelerator pulse arrives. The acquisition, peak analysis and transfer process would then be repeated for each accelerator pulse until sufficient data is accumulated.

Each detection channel would have its own detector and PMT, front-end electronics and flash ADC; however, the buffer memory can be common to multiple detection channels and the AIM can support two buffer memory inputs. The fast front-end electronics and logic control circuits would have to be specifically designed for this apjlication, but the flash ADC, AIM, MCA and personal computer are all commercially available. The basic acquisition and analysis software would also be commercially available. 


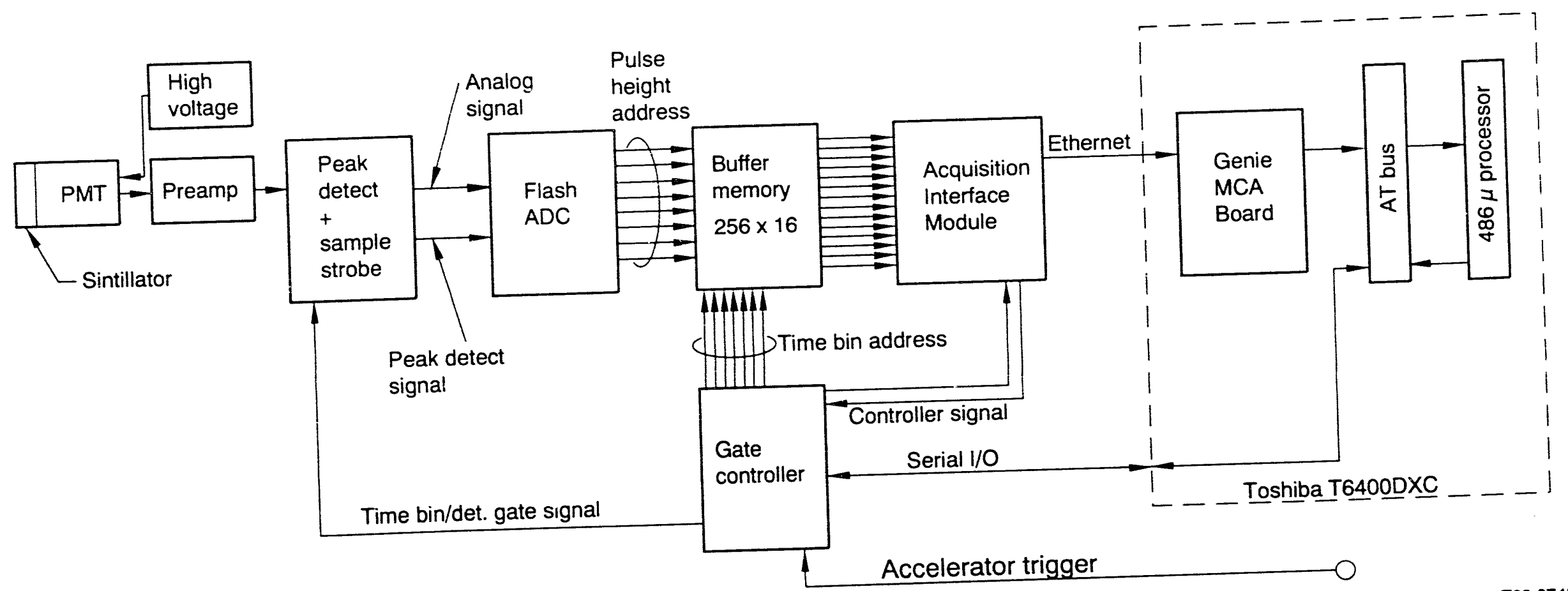

Figure 6.1 Fast detection and acquisition system. 
The initial detection system would have three detection channels to support data acquisition in three time regions following an accelerator burst; e.g., 30 to $150 \mathrm{ns,}$ 150 to $500 \mathrm{~ns}$ and $500 \mathrm{~ns}$ to $5 \mu \mathrm{s}$. The time boundaries indicated are nominal values based on the separation of inelastic and capture events and the improved response of candidate detector materials. 


\section{RECOMMENDATIONS}

Based on the preliminary numerical predictions and the experimental temporal response performance of candidate gamma detectors, basic concept feasibility has been shown. It is recommended that continued support of this interrogation concept should include the following multi-year tasks:

- development of a preliminary fast detection system capable of gamma pulse height detection and processing for at least one pulse for each accelerator pulse (may require Compton edge deconvolution methods for spectral analyses),

- extension of the fast detection system development to enable multiple gamma pulse height detection and processing at rates up to $40 \mathrm{MHz}$ per accelerator pulse (as described in Section 6),

- numerical and experimental system optimization of the electron beam current (i.e., pulse width and intensity) and the detection system temporal response and energy resolution.

- proof-of-concept testing at Santa Barbara Linear Accelerator Facility using selected counter-proliferant, object materials and candidate detectors,

- extension of proof-of-concept operations/results to compact, commercial accelerator designs,

- development of a complete prototype system, and

- system demonstration. 


\section{REFERENCES}

1. S. H. Vegors, Jr. and E. B. Nieschmidt, "Preliminary investigation of a Criticality Monitoring Technique for a Transuranic Waste Incinerator," INEL Report, TREE-1285, October 1978.

2. J. R. Lamarch, Introduction to Nuclear Reactor Theory, Addison-Weslely Publishing Co., Mass., 1972.

3. R. D. Evans, The Atomic Nucleus, McGraw-Hill Book Co., New York, 1955.

4. S. S. Dietrich and B. L. Berman, Atlas of Photoneutron Cross Sections Obtained with Monoenergetic Photons, UCRL-94820, June 1986.

5. J. L. Jones, et al, "Gamma-Neutron Threshold (GNT) Technique: Proof-ofConcept Experiment Description and Results (U)," [SECRET] INEL Report AA2668, February 1991.

6. J. L. Jones, et al, "Gamma-Neutron Threshold (GNT) Technique: FY-91 Yearend Report and Follow-On Experimental Results (U)," [SECRET] INEL Report AA-2728, October 1991.

7. F. Everling, et al, 1960 Nuclear Data Tables Parts 1 and 2, Consistent Set of Energies Liberated in Nuclear Reactions, NAS 1961.

8. J. R. Lamarch, Introduction to Nuclear Engineering, Addison-Weslely Publishing Co., Mass., 1983.

9. Radiation Protection Design Guidelines for 0.1-100 MeV Particle Accelerator Facilities, National Council on Radiation Protection and Measurements (NCRP Report No. 5!), March 1977.

10. I. B. Berlman, et al, "New Fast Organic Scintillators Using Intramolecular Bromine Quenching," Nuclear Instruments and Methods in Physics Research, 225, (1984) 78-92.

11. C. B. Asford, et al, "High-Z Liquid Scintillators Containing Tin," Nuclear Instruments and Methods in Physics Research, A243, (1986) 131-136.

12. L. O. John'son, "Evaluation of Candidate Scintillator Detectors,: INEL-EDFRML001, Project/Task MIT-1, January 1993.

13. L. O. Johnson, "FY-93 Evaluation and Design Results," INEL-EDF-RML002, Project/Task SW001, May 1993. 
14. J. A. Hablieb and T. A. Mehlhorn, ITS: The Integrated TIGER Series of Coupled Electron/Photon Monte Carlo Transport Code, SNL, SAND84-05873, November 1984.

15. J. F. Briesmeiter editor, MCNP - A general Monte Carlo Code for Neutron and Photon Transport, LANL, LA-7396 (Rev 2), September 1986

16. S. Budavari, ed., The Merck Index, Eleventh Edition Rahway, N.J: Merck \& Co., Inc., 1989.

17. J. H. Hubbell, Photon Cross Sections, Attenuation Coefficients, and Energy Absorption Coefficients from $10 \mathrm{keV}$ to $100 \mathrm{GeV}$, National Bureau of Standards, NSRDS-NBS 29, August 1969.

18. J. L. Jones letter to S. J. Fernandez, "Temporal Response Assessment of MIT Candidate Fast Scintillators," INEL Letter JLJ-9-93, April 30, 1993.

19. A.J. Caffrey, et al, "Chemical Warfare Agent and High Explosive Identification by Spectroscopy of Neutron-induced Gamma Rays, "IEEE Transactions on Nuclear Science, Vol. 39, 1992, pp 1422-1426.

20. A. Beyerle, et al, "Design of an associated particle imaging system," Nuclear Instruments and Methods in Physics Research, Vol. A299, 1990, pp 458-462.

21. M. F. Physics Inc, Vendor data sheet.

22. M. L. O'Neal, MA165C Zetatron Neutron Generator - Operator Manual, Sandia National Laboratory Report, SAND85-0152, August 1990.

23. J. A. Harvey, Experimental Neutron Resonance Spectroscopy, Academic Press, New York, 1970. 


\section{APPENDIX}

INEL written program (Photon Induced Neutron Production - PINP) used to calculated photoneutron and photofission neutron production in modeled regions with specified photon fluxes. The program is written in FORTRAN-7 and the listing follows.

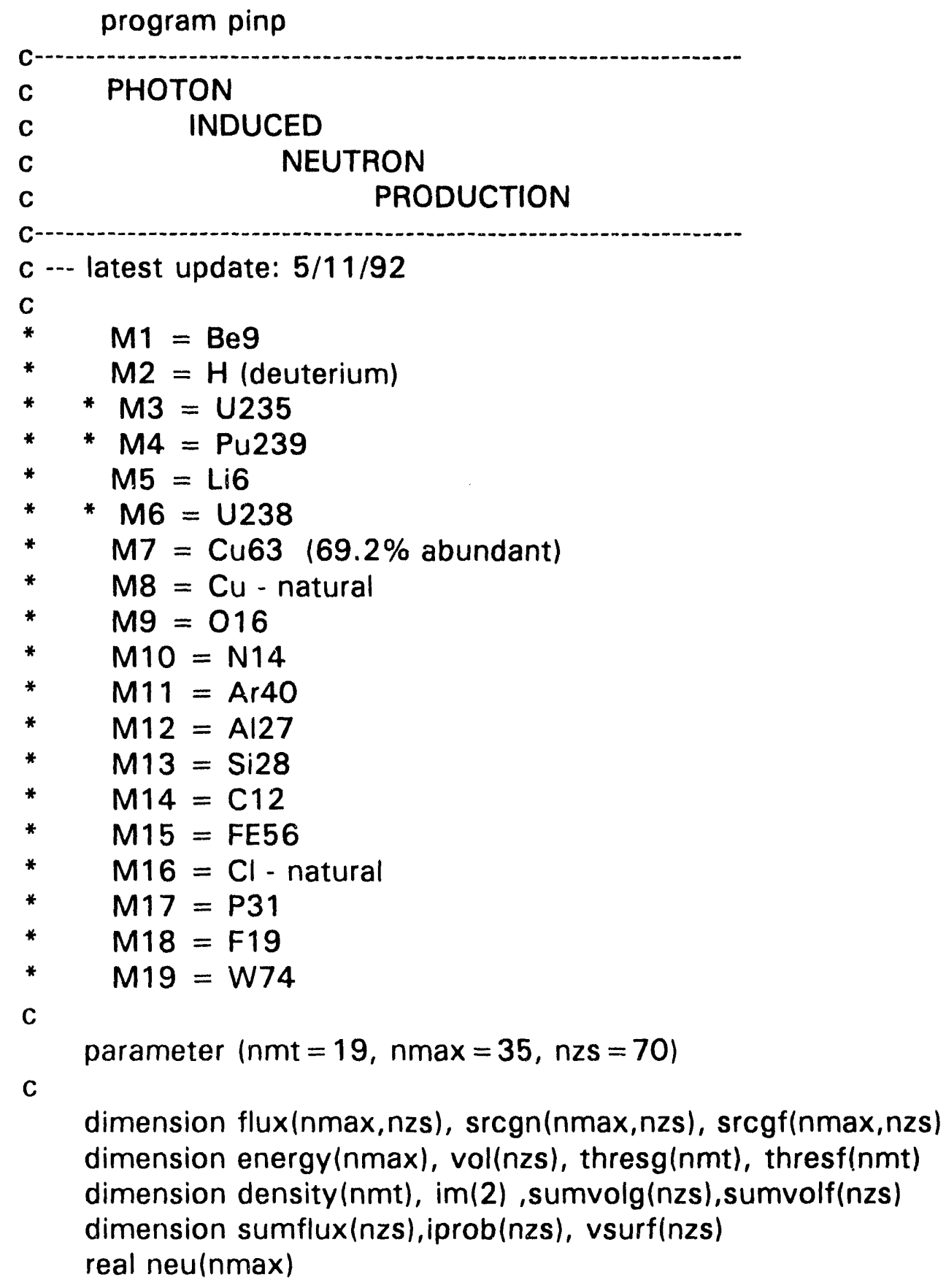




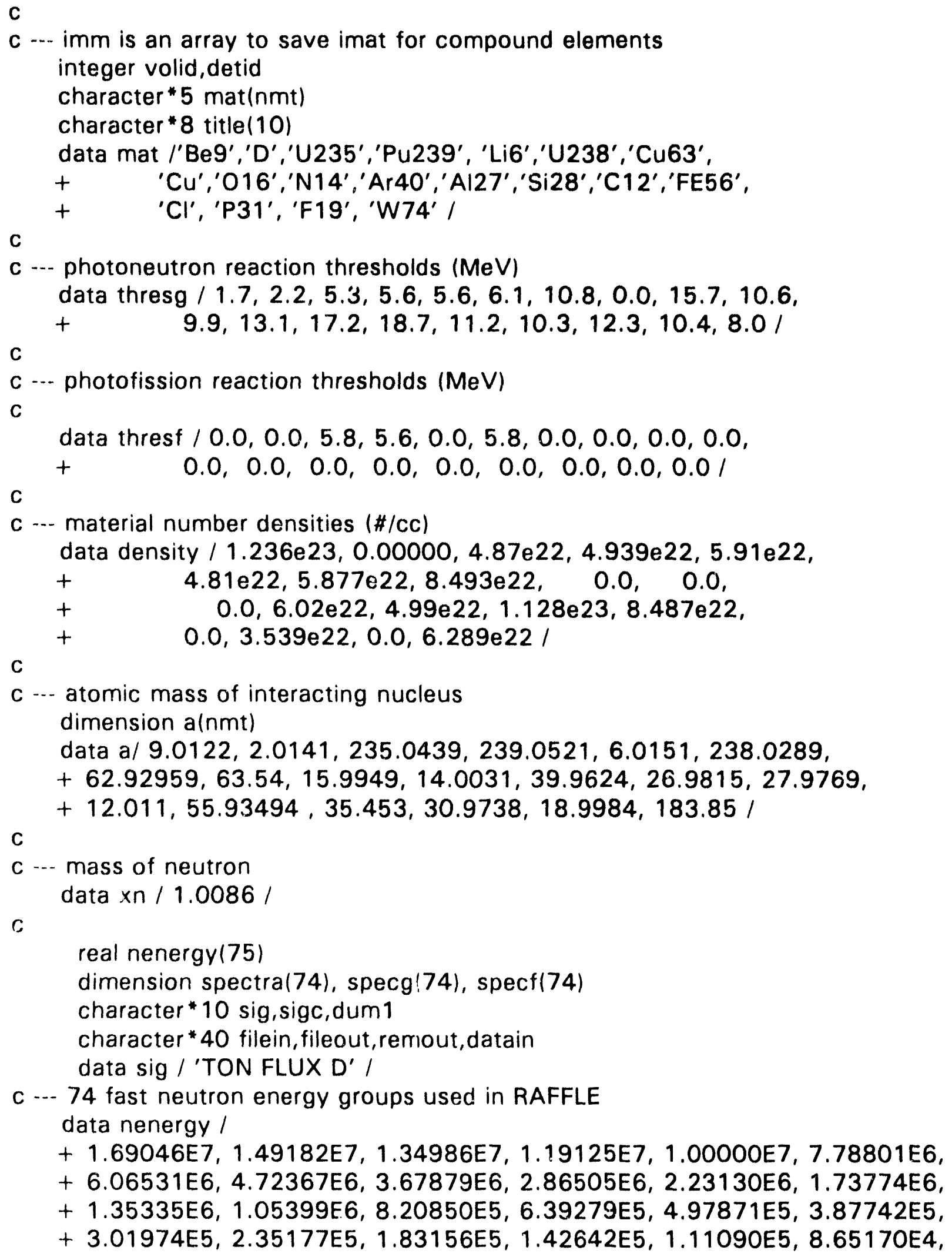


$+6.73795 \mathrm{E} 4,5.24752 \mathrm{E} 4,4.08677 \mathrm{E} 4,3.18278 \mathrm{E} 4,2.47875 \mathrm{E} 4,1.93045 \mathrm{E} 4$,

$+1.50344 \mathrm{E} 4,1.17088 \mathrm{E} 4,9.11882 \mathrm{E} 3,7.10174 \mathrm{E} 3,5.53084 \mathrm{E} 3,4.30743 \mathrm{E} 3$,

$+3.35463 E 3,2.61259 E 3,2.03468 E 3,1.58461 E 3,1.23410 E 3,9.61117 E 2$,

$+7.48518 E 2,5.82947 E 2,4.53999 E 2,3.53575 E 2,2.75364 E 2,2.14454 E 2$,

$+1.67017 \mathrm{E} 2,1.30073 \mathrm{E} 2,1.01301 \mathrm{E2}, 7.88932 \mathrm{E} 1,6.14421 \mathrm{E} 1,4.78512 \mathrm{E} 1$,

$+3.72665 \mathrm{E} 1,2.90232 \mathrm{E} 1,2.26033 \mathrm{E} 1,1.76035 \mathrm{E} 1,1.37096 \mathrm{E} 1,1.06770 \mathrm{E} 1$,

$+8.31529,6.47595,5.04348,3.92786,3.05902,2.38237$,

$+1.85539,1.44498,1.12535,8.76425 \mathrm{E}-1,6.82560 \mathrm{E}-1,5.31579 \mathrm{E}-1$,

$+4.13994 \mathrm{E}-1, \quad 0.02, \quad 0.0 /$

C

C

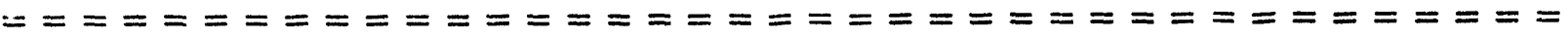



c read input file

C

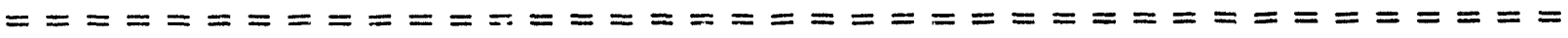



C

print *' Read INPUT from a data file? $(0=\text { no, } 1=y e s)^{\prime}$

read *, iians

C

c-.- batch mode

C

if( iians .eq. 1 ) then

print * ', INPUT file name (in single quotes)'

read * datain

open (unit $=2$, file $=$ datain, status $=$ 'old $^{\prime}$ )

$\operatorname{read}\left(2,{ }^{*}\right)$

read $\left(2,{ }^{*}\right)$ filein

open (unit $=5$, file $=$ filein, status $={ }^{\prime}$ old ${ }^{\prime}$ )

$\operatorname{read}\left(2,{ }^{*}\right)$

read $(2, *)$ energy $(1)$

read $(2, *)$

read $(2, *)$ neg

read $(2, *)$

read $(2, *)$ ntvol

read $(2, *)$

read $(2, *)$ volid

read $(2, *)$

read $\left(2,,^{*}\right)$ matid

read $\left(2,{ }^{*}\right)$

read $\left(2,{ }^{*}\right)$ volume

read $(2, *)$

read $(2, *)$ ians 




22 print * ,'Enter the OUTPUT file name (in single quotes)' read *, fileout

C 
endif

C

c --- determine the appropriate number density dense $=\operatorname{density}($ matid)

if (ians .eq. 1 ) dense $=$ tmwtf $^{*}$ tdens ${ }^{*} 6.023 e 23 /$ a $($ matid $)$

C

open (unit $=6$, file $=$ fileout, status $=$ 'unknown', err $=22$ )

C

write $(6,27)$ filein, fileout,energy $(1)$, neg, ntvol,volid,

$\& \quad$ mat(matid), volume,thresg(matid), thresf(matid),

$\&$ dense

27 format(1h, 'INPUT: ',a40,' OUTPUT: ',a40, /,

\& 'Max. E (MeV): ',1pe10.4,' No. of E groups ',i5,

$\&$ 'Tot. Vols: ',i5,/,

\& ' Target Vol.-of-Interest: No. $=$ ',i3,' ID = ',a5

$\&,^{\prime} \mathrm{CM}=$ ',1 pe10.4,' Ethres $(g, n)(\mathrm{MeV})=$ ', 1 pe10.4

\& ' Ethres $(g, f)(\mathrm{MeV})=$ ',1 pe 10.4,

$\& 1,25 x, '$ Number Density $(\# / c c)=', 1$ pe 10.4)

C

if( ians .eq. 1 ) write $(6,28)$ tdens, tmwtf

28 format(1h,'COMPOUND DATA: Density (g/cc) =', 1pe 12.4,

${ }^{\prime}$ Mol. wt fraction $=$ ', 1 pe 12.4)

C

negy $=$ neg +1

C

C

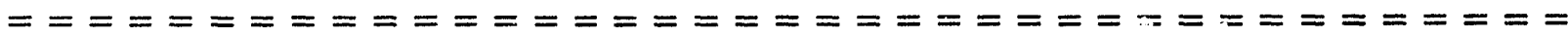

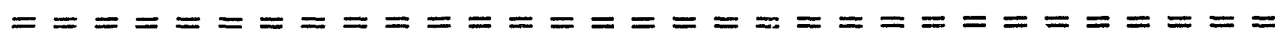

c -.- read in flux here.. tape 5 is the accept output file.

C



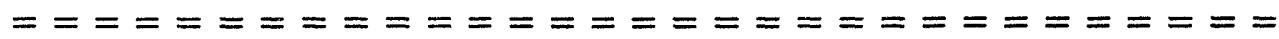

C

write $(6,370)$

370 format(1h,' Photon flux distribution ',/)

C

c -.- look for photon flux distribution

iadd $=9$

istrt $=1$

ichk $=$ ntvol $/ 10$

if( ichk .It. 1 ) iadd $=$ ntvol -1

ilast $=$ istrt + iadd

c

$100 \operatorname{read}(5,110$, end $=999)$ sigc 


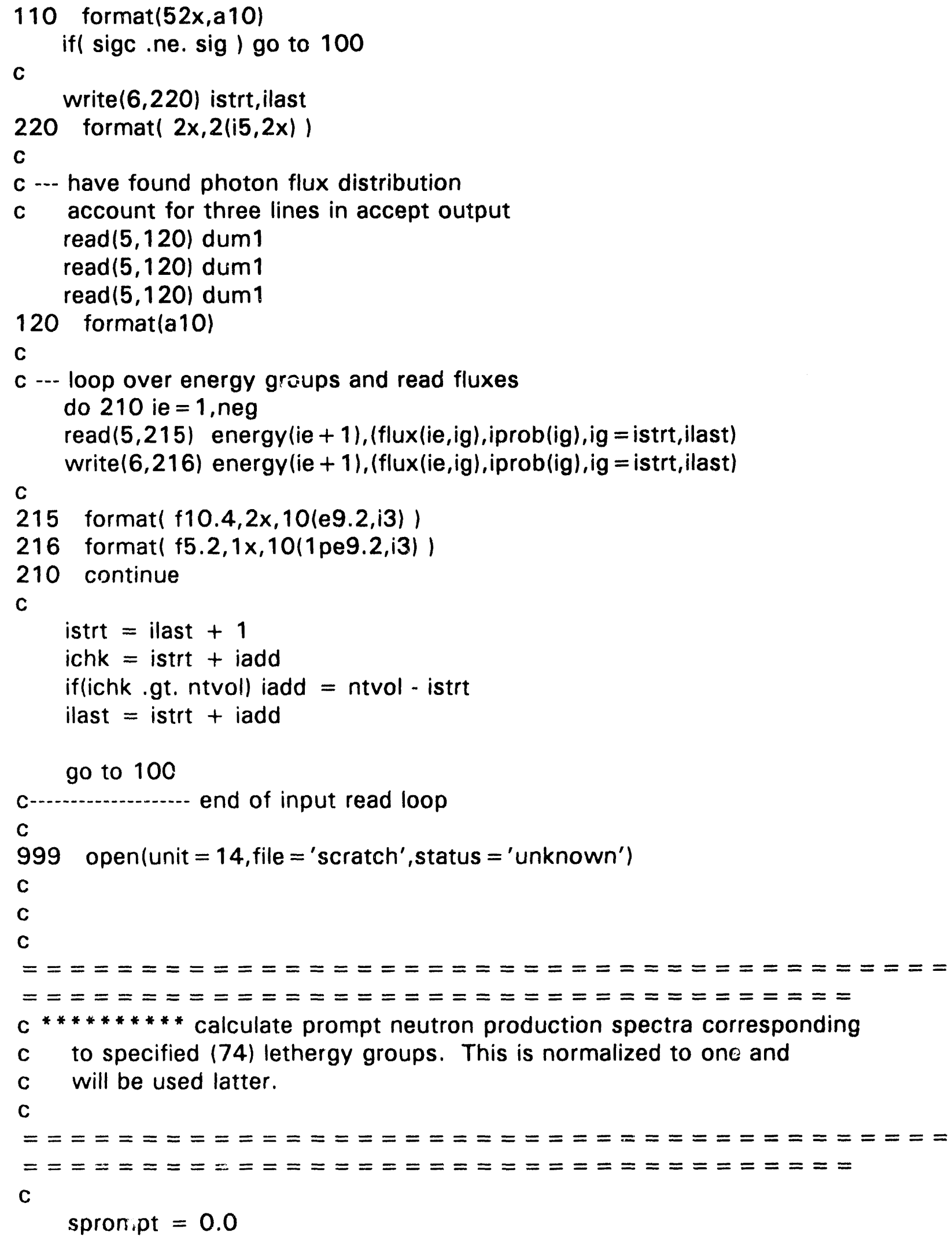


do 250 in $=1,74$

c --- convert to $\mathrm{MeV}$ from ev

$\mathrm{e} 1=$ nenergy $(\mathrm{in}) / 1 . \mathrm{e} 6$

$\mathrm{e} 2=$ nenergy $(\mathrm{in}+1) / 1 . \mathrm{e} 6$

call prompt (e1,e2, spectra(in))

sprompt $=$ sprompt + spectra(in)

250 continue

C

C



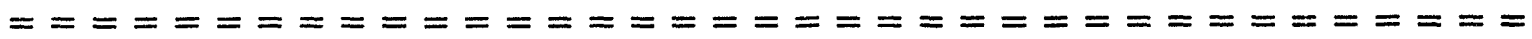

C C $^{* * * * * * * * *}$ convolute photon spectra with cross-sections



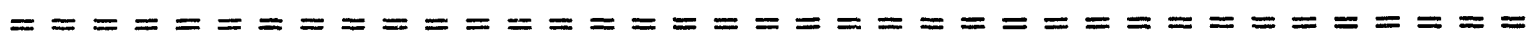

c -.- loop over zones or volumes

C

$\mathrm{k}=$ matid

dose $=0.0$

do $400 \mathrm{iz}=1$, ntvol

C

C

sumvolg(iz) $=0.0$

sumvolf(iz) $=0.0$

sumflux (iz) $=0.0$

sumdele $=0.0$

sumneu $=0.0$

C

c -.- loop over energy groups

do 410 ie $=1$, neg

$\operatorname{srcgn}(i e, i z)=0.0$

C

dele $=\operatorname{energy}(i e)-\operatorname{energy}(i e+1)$

sumflux $(i z)=$ sumflux $(i z)+$ flux $(i e, i z){ }^{*}$ dele

C

if( iz . ne. volid ) go to 410

C

c -.- calculate gamma dose in volume-of-interest

eavg $=($ energy $(i e)+\operatorname{energy}(i e+1)) / 2.0$

call doseg (eavg,dfg)

c ... dose in rads

dose $=$ dose $+\mathrm{dfg}^{*}$ flux $(i e, i z)^{*}$ dele $/ 3600$.

c

C

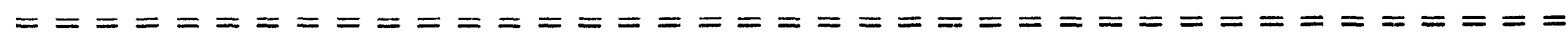




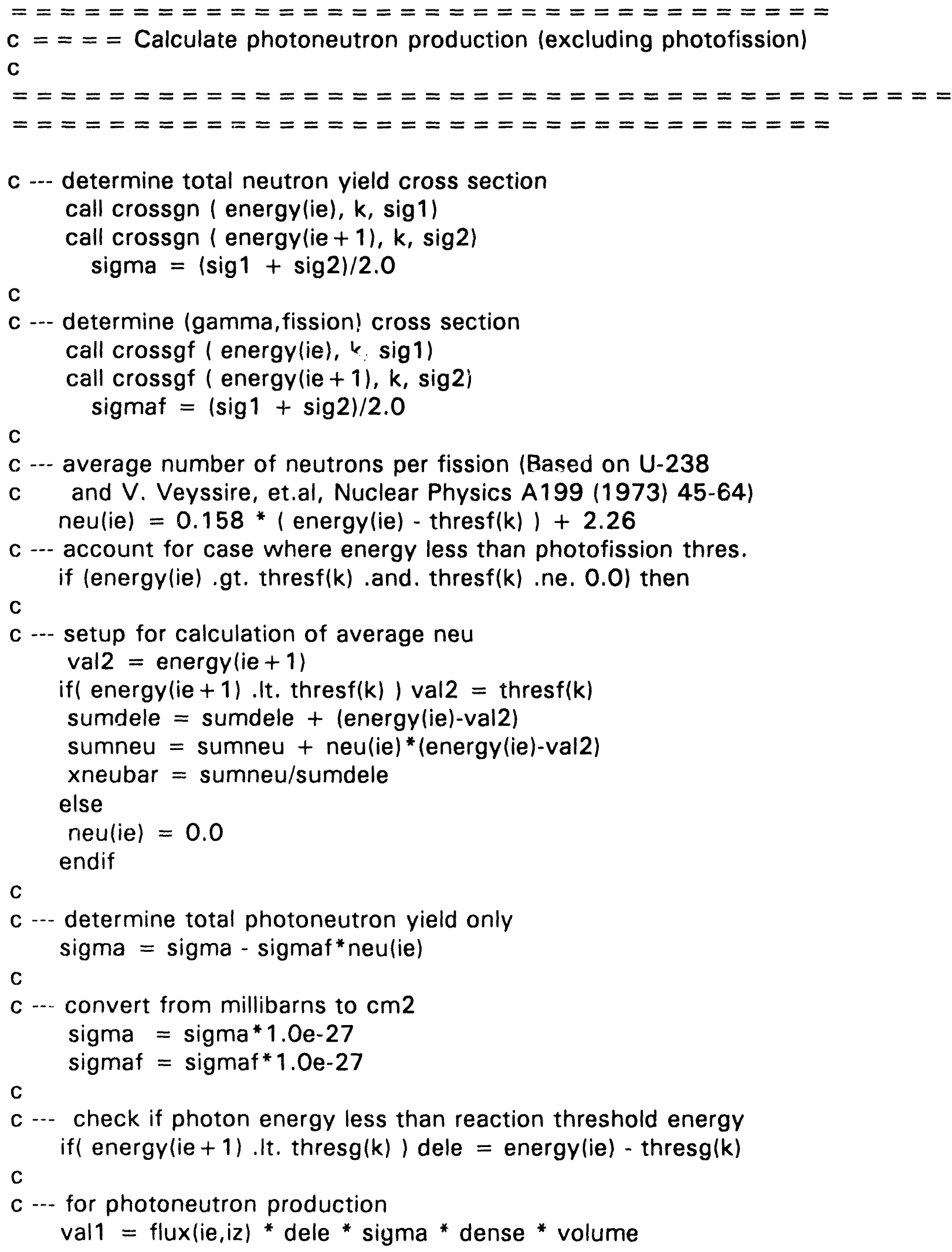


if ( val1 .It. 0.0$)$ val1 $=0.0$

$\operatorname{srcgn}(i e, i z)=\operatorname{srcgn}(i e, i z)+$ val1

sumvolg(iz) $=$ sumvolg $(i z)+\operatorname{srcgn}(i e, i z)$

C

C --- approximate neutron energy range based on mono-energetic gamma group

c enh and enl in eV's

C

val1 $=1.0 \mathrm{e} 6^{*}(\mathrm{a}(\mathrm{k})-\mathrm{xn}) / \mathrm{a}(\mathrm{k})$

enh $=$ val ${ }^{*}\left(\right.$ energy $(i e)$-thresg(k)-energy $\left.(i e)^{* *} 2 /\left(1862 .{ }^{*}(a(k)-x n)\right)\right)$

enl $=$ val $1 *($ energy $(i e+1)$-thresg $(k)$-energy $($ ie +1$) * * 2$

$\left.1 /\left(1862{ }^{*}(a(k)-x n)\right)\right)$

c

eno $=$ enh

c --- check if enh is less than lowest energy group

if( enh .le. 0.02 ) then

eno $=0.02$

go to 305

endif

c -.- check if enl is less than lowest energy group

if( enl .It. 0.02 ) then

enl $=0.02$

endif

C

c --- calculate \# of source neutrons per eV

$s 1=\operatorname{srcgn}(i e, i z) /(e n h-e n l)$

C

do 300 in $=1,74$

if( nenergy(in). It. enh) then

if( nenergy(in) .gt. enl) then

den $=$ enh - nenergy (in)

$\operatorname{specg}($ in $)=\operatorname{specg}($ in $)+s 1^{*}$ den

enh $=$ nenergy $(\mathrm{in})$

else

den $=$ enh - enl

$\operatorname{specg}($ in $)=\operatorname{specg}($ in $)+s 1 *$ den

go to 305

endif

endif

300 continue

c

305 continue

c

C

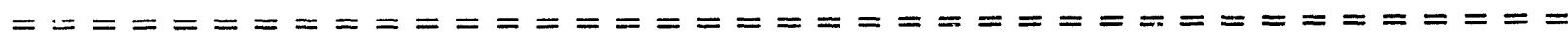




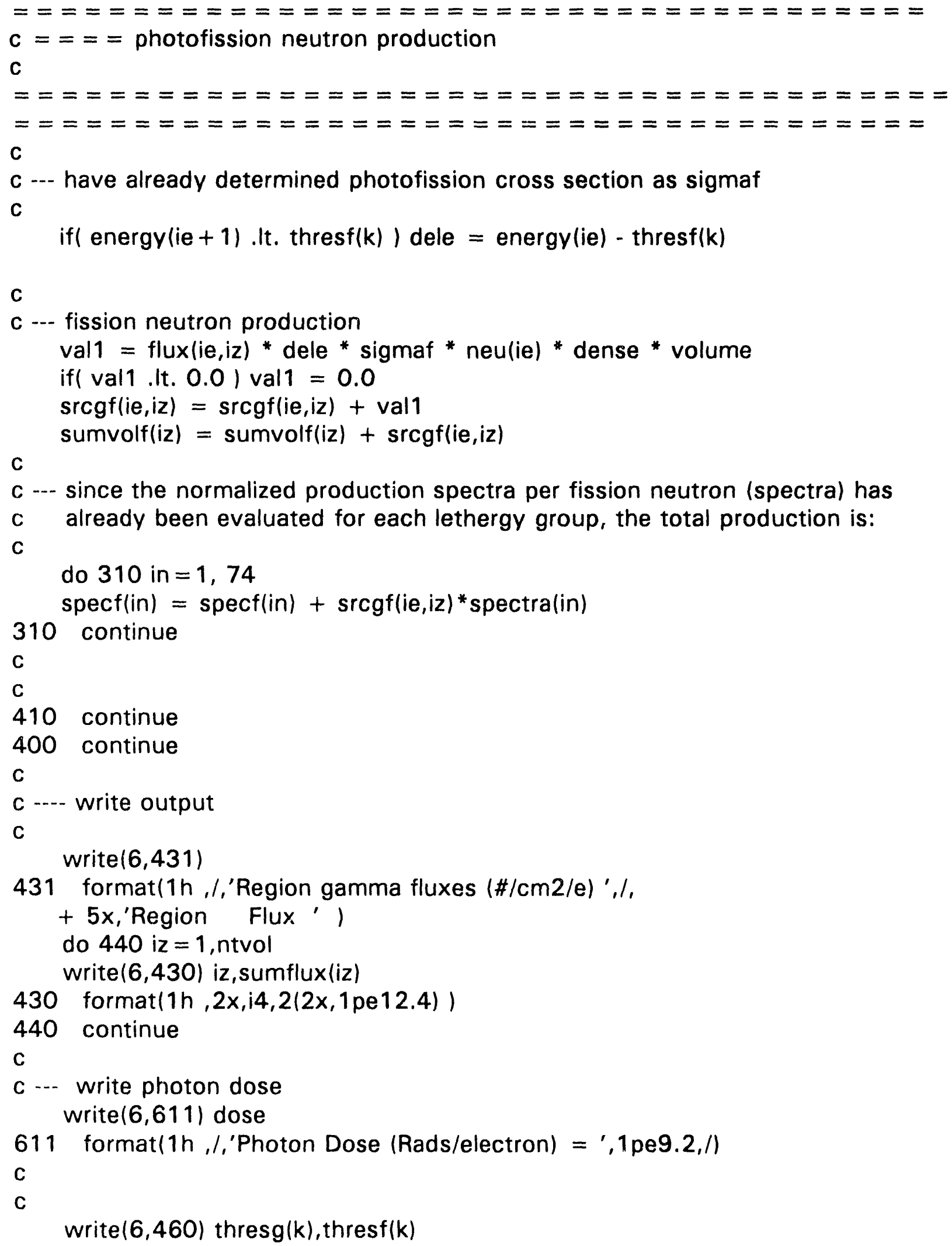


460 format $(1 \mathrm{~h}, /, \mathrm{N}$ production per incident electron', $\&^{\prime}$ in Volume-of-Interest', /, 2x,

$\&^{\prime}$ Thres. Energy $(\mathrm{MeV})=', 1$ pe12.4,4x,1 pe12.4,/,16x,

$\left.\&^{\prime}(\mathrm{MeV}) \quad(g, n) \quad(g, f) \quad \mathrm{Neu}, / /\right)$

C

499 do 500 ie $=1$, neg

write $(6,510)$ energy(ie +1$)$, srcgn(ie,volid), srcgf(ie,volid),

1 neu(ie)

510 format $(1 \mathrm{~h}, 13 \mathrm{x}, \mathrm{f} 10.4,5 \mathrm{x}, 10(1 \mathrm{pe} 9.2,5 \mathrm{x})$ )

500 continue

write $(6,511)$ sumvolg(volid), sumvolf(volid)

511 format $\left(1 \mathrm{~h}, /,{ }^{\prime}\right.$ Total (neutron/electron) $=', 2(1$ pe9.2, $\left.5 x), /\right)$

C

write $(6,1051)$ sprompt

1051 format(1h ,'Integrated normalized prompt ',

+ 'fission spectra $=$ ', 1 pe 12.4)

C

c -- neutron intensity spectrum results

C

467 format $\left(/ / /, 3 x_{,}{ }^{\prime G P}, 6 x,{ }^{\prime} \text { Neutron',8x, }(g, n)\right)^{\prime}, 6 x,{ }^{\prime}$ ',

$+6 x_{1}^{\prime}(g, f)^{\prime}, 6 x^{\prime}{ }^{\prime}$, ,

$\left.+6 x^{\prime}(\mathrm{g}, \text { total) })^{\prime}, 6 \mathrm{x},{ }^{\prime}{ }^{\prime}\right)$

468 formatl $3 x^{\prime}{ }^{\prime}$ ',6x,'Energy (ev) ', 3x, 'Source $(n / e)^{\prime}, 1 x,{ }^{\prime} \%$ ',

$+6 x^{\prime}$ 'Source $(n / e)^{\prime}, 1 x^{\prime} \%{ }^{\prime}$,

$+6 x^{\prime}$ 'Source $\left.(n / e)^{\prime}, 1 x^{\prime}, \%^{\prime}\right)$

469 FORMAT( $3 X, 12,4 X, 1$ pe $11.5,3 x, 3(1 \mathrm{PE} 11.5,3 \mathrm{X}, 2 \mathrm{pf} 5.2,2 \mathrm{x})$ )

C

C

c --- must account for the case where no photoneutrons are produced

C

sumg $=0.0$

sumge $=0.0$

sumf $=0.0$

sumfe $=0.0$

C

do $1050 \mathrm{jj}=1,74$

$\mathrm{de}=($ nenergy $(j \mathrm{j})+$ nenergy $(\mathrm{jj}+1) / 2.0$

surnge $=$ sumge + specg $(j j){ }^{*}$ de

sumg $=$ sumg + specg $(j \mathrm{j})$

sumfe $=$ sumfe $+\operatorname{specf(jj)})^{*} d e$

sumf $=\operatorname{sumf}+\operatorname{specf}(j \mathrm{j})$

C

1050 continue 


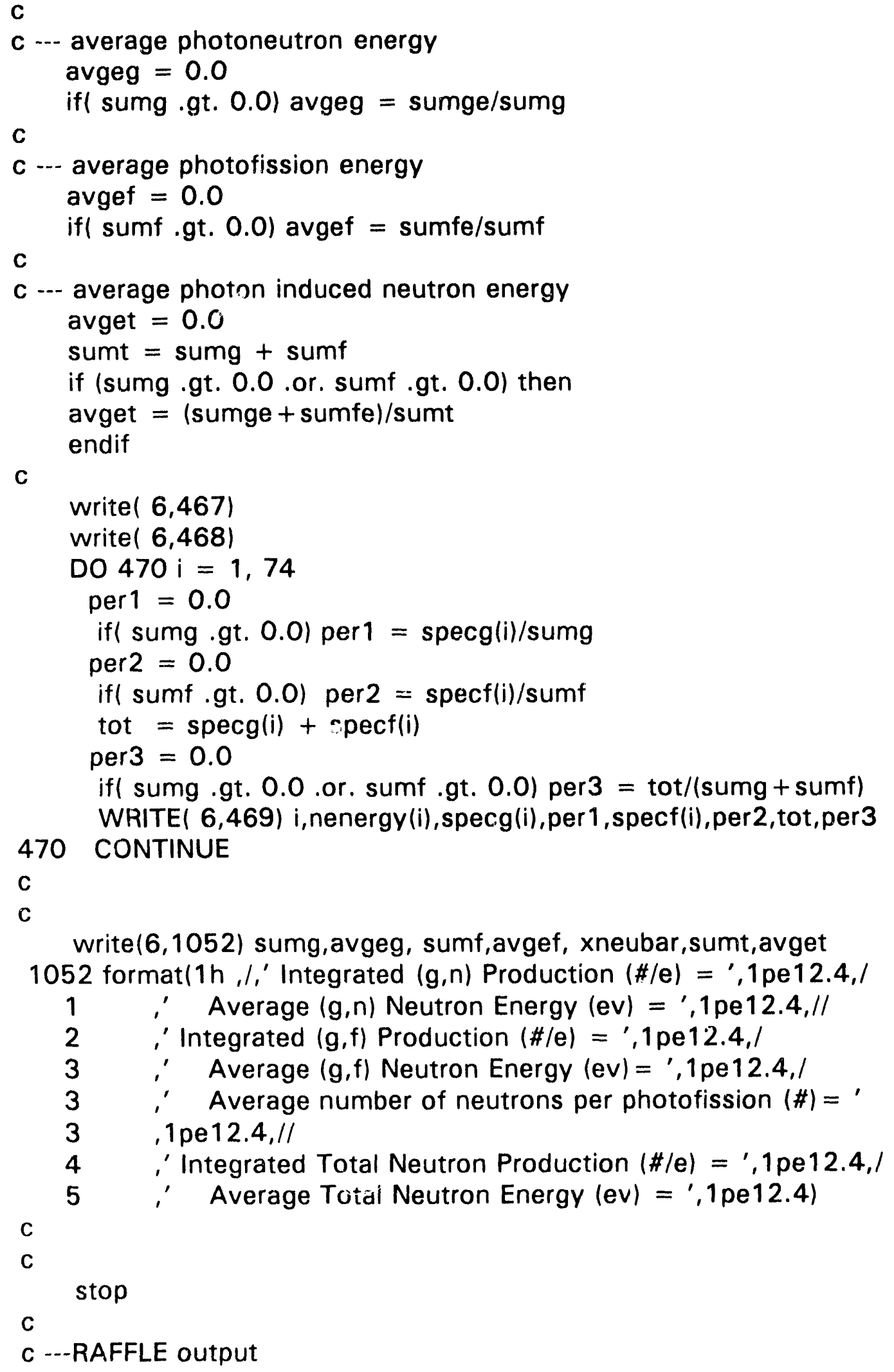


c

open ( unit $=7$, file $=$ 'ns. raf' $^{\prime}$

number $=74$

$\mathrm{nn}=$ number $/ 5$

$i 5=5$

isp $=1$

C

C --- set up fractional intensity distributions

in $=1$

do $2000 i=1, \mathrm{nn}$

write $(7,2001)$ isp, in, isp, i5, (specg $(i n+j), j=1, i 5)$

2001 format $\left(1 \mathrm{~h},{ }^{\prime} 6^{\prime}, i 2, i 3, i 2, i 2,5(1 \mathrm{pe}\right.$ 12.4) )

in $=$ in +5

2000 continue

iend $=$ number - in

write $(7,2001)$ isp, in, isp, iend, $(\operatorname{specg}(i n+j), j=1$,iend $)$

C

C

c --- set up energy matrix for transfer to raffle

in $=1$

do $2100 i=1, n n$

write $(7,2101)$ in, $i 5$, (nenergy $($ in $+j), j=1, i 5)$

2101 format $\left(1 \mathrm{~h},{ }^{\prime}, ', 3,2 x, i 2,5(1 \mathrm{pe} 12.4)\right.$ )

in $=$ in +5

2100 continue

c

iend $=$ number - in

write $(7,2101)$ in, iend, (nenergy $(i n+j), j=1$, iend)

C

stop

end

C

subroutine doseg ( eavg, dfg )

C

C --- routine to calculate gamma dose rate conversion factor: $\mathrm{dfg}$

C

C dose $(\mathrm{rad} / \mathrm{hr})=\mathrm{dfg}{ }^{*}$ gflux $(\# / \mathrm{cm} / \mathrm{s})$

C

C -.- form of the equation:

$c \quad \ln (d f g)=a+b \ln ($ eavg $)+c \ln (\text { eavg })^{* *} 2+d \ln (\text { evag })^{* *} 3$

dimension $a(4), b(4), c(4), d(4), e(4)$

C

low-0.03 $0.03-0.5 \quad 0.5-5$.

c 


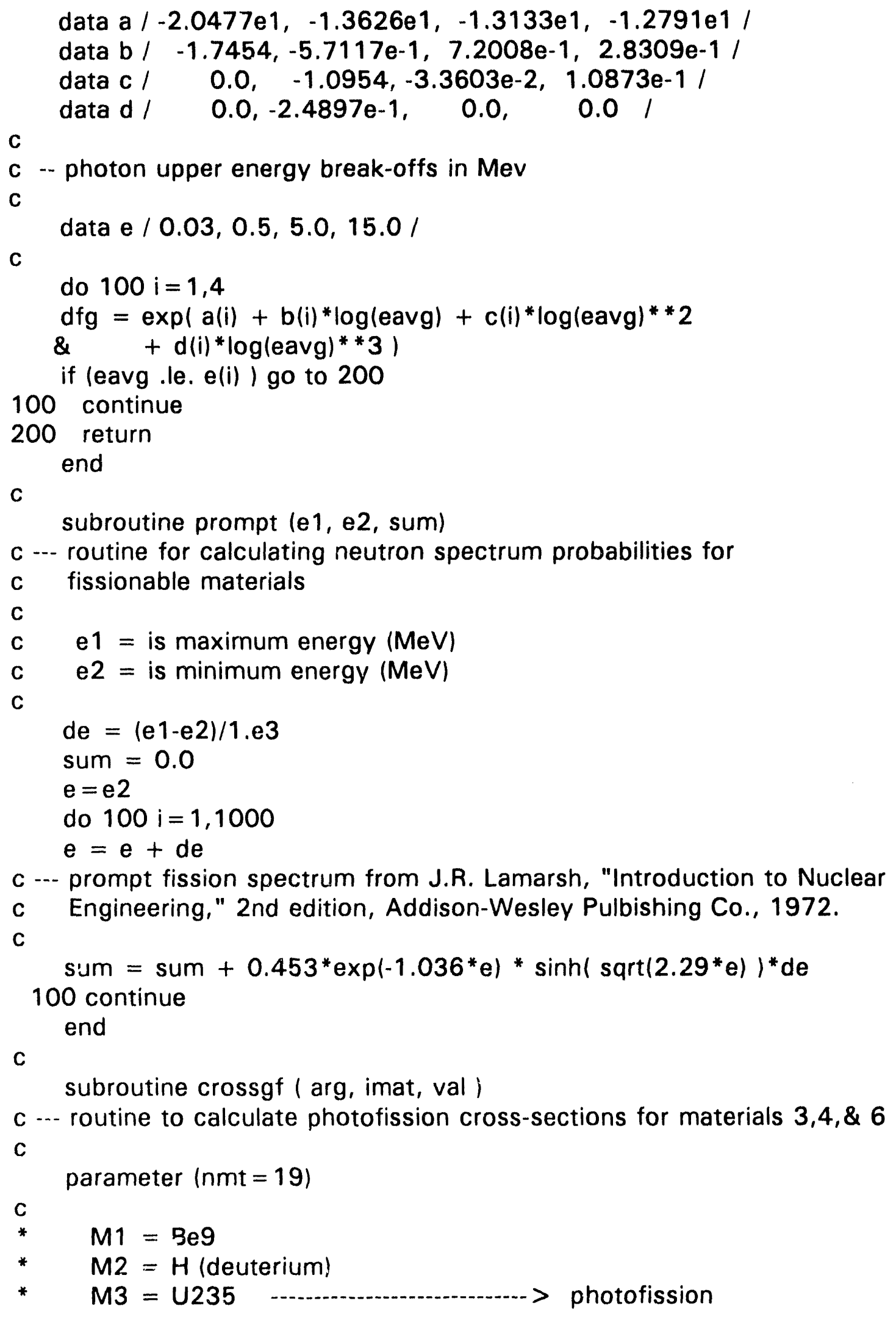




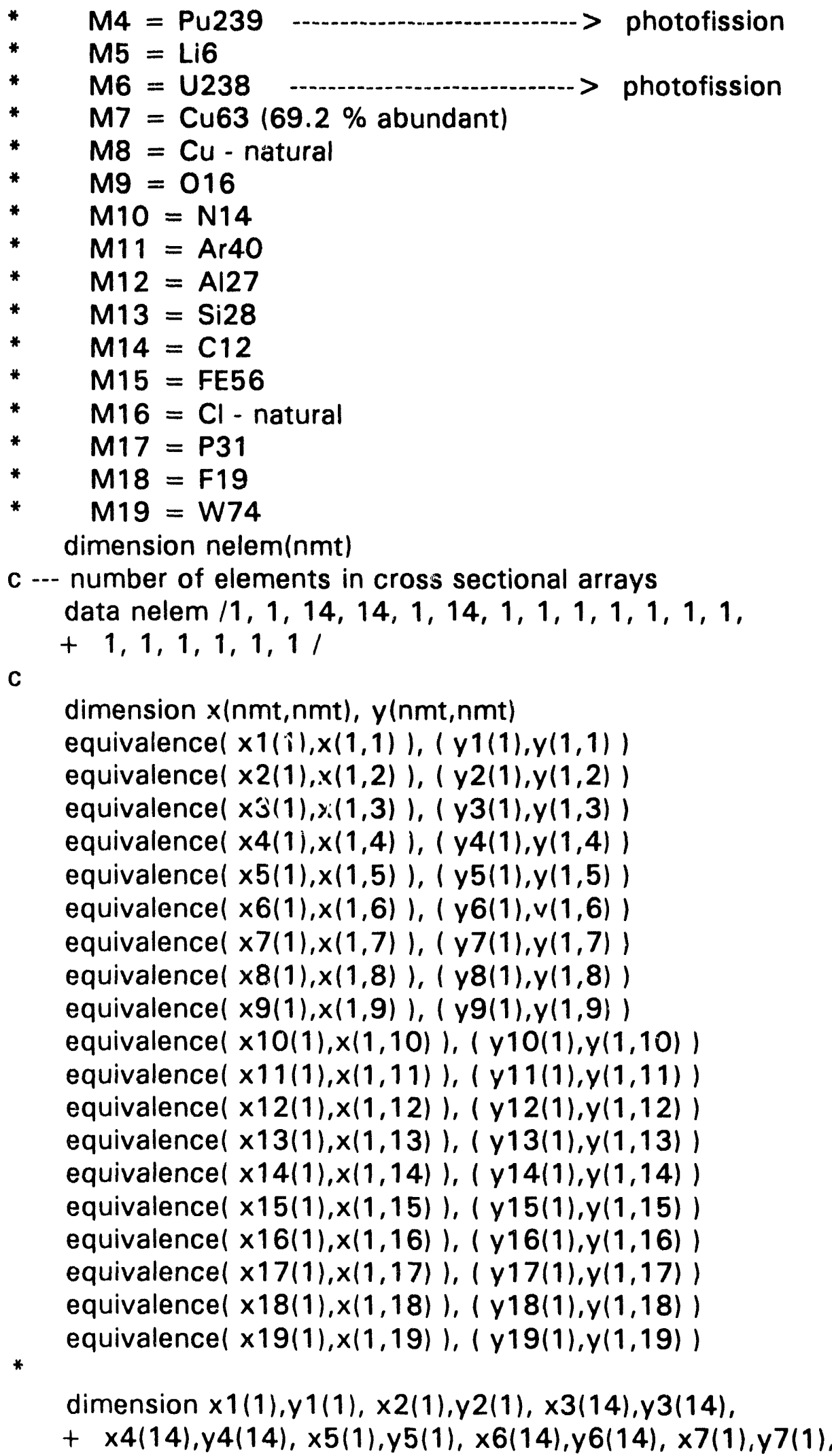


$+x 8(1), y 8(1), x 9(1), y 9(1), x 10(1), y 10(1), x 11(1), y 11(1)$,

$+x 12(1), y 12(1), x 13(1), y 13(1), x 14(1), y 14(1)$,

$+x 15(1), y 15(1), x 16(1), y 16(1), x 17(1), y 17(1)$,

$+x 18(1), y 18(1), x 19(1), y 19(1)$

$* 1$

* Be

DATA $\times 1 / 0.0 /$

DATA Y $1 / 0.0 /$

$* 2$

* H 2 (DEUTERIUM)

DATA $\times 2 / 0.0 /$

DATA Y $2 / 0.0 /$

* 3

* U 235 SS Dietrich LLNL 1986 (photofission cross section)

* photofission energy threshold $=5.8 \mathrm{MeV}$

DATA X3/5.8, 6., 7., 8., 9., 10., 11., 12., 13., 14.,

$+\quad 15 ., 16 ., 17 ., 18.1$

DATA Y3/0.0, 8., 15., 25., 60., 120., 200., 240., 360., 400.,

$+\quad 310 ., 220 ., 150 ., 100.1$

* 4

* PU 239 SS Dietrich LLNL 1986 (photofission cross section).

* photofission energy thres $=5.6 \mathrm{Mev}$, photoneutron energy thres $=5.6 \mathrm{MeV}$

DATA X4 / 5.6, 6.0, 7.0, 8.0,9.0, 10.0, 11.0, 12.0, 13.0, 14.0,

$+\quad 15.0,16.0,17.0,18.0 /$

DATA Y4 / 0.0, 5. , 20., 35., 60., 150., 250., 290., 300., 360.,

$+\quad 300 ., 200 ., 150 ., 80.1$

$* 5$

* Li 6

DATA $\times 5 / 0.0 /$

DATA y $5 / 0.0 /$

* 6

* U 238 SS Dietrich LLNL 1986 (photofission).

* photofission energy thres $=5.8 \mathrm{Mev}$

DATA X6/5.8, 6., 7., 8., 9., 10., 11., 12., 13., 14., $+\quad 15 ., 16 ., 17 ., 18.1$

DATA Y6/0.0, 8., 11., 15., 30., 68., 102., 95., 145., 170.,

$+\quad 160 ., 120 ., 90 ., 74.1$

$* 7$

* Cu 63 (69.3\% abundant) (MeV \& mb * 11/90)

data $\times 7 / 0.0 /$

data $y 7 / 0.0 /$

$* 8$

* dummy

data $\times 8 / 0.0 /$ 
* 9

data y8/0.0/

* 016 ( yield cross section: MeV \& mb )

DATA X9/0.0/

DATA Y9/ $0.0 /$

* 10

* N 14 ( yield cross section: MeV \& mb )

DATA X10/0.0/

DATA Y10/ $0.0 /$

* 11

* Ar 40 (yield cross section: MeV \& mb )

DATA $\times 11 / 0.0 /$

DATA Y11/0.0/

* 12

* Al 27 (yield cross section: MeV \& mb )

DATA $\times 12 / 0.0 /$

DATA Y12/0.0/

* 13

* Si 28 (yield cross section: MeV \& mb )

DATA X13/0.0/

DATA Y13/0.0/

* 14

* C 12 (yield cross section: MeV \& mb ) data $\times 14 / 0.0 /$

data y $14 / 0.0 /$

* 15

* FE 56 (yield cross section: MeV \& mb ) data $\times 15 / 0.0 /$ data $y 15 / 0.0 /$

* 16

* $\mathrm{Cl}$ natural (yield cross section: MeV \& mb ) data $\times 16 / 0.0 /$ data $y 16 / 0.0 /$

* 17

* P31 (yield cross section: MeV \& mb ) data $\times 17 / 0.0 /$ data $y 17 / 0.0 /$

* 18

* F19 (yield cross section: MeV \& mb ) data $\times 18 / 0.0 /$ data $>18 / 0.0 /$

* 19

* W74 (yield cross section: MeV \& mb ) data $\times 19 / 0.0 /$ 
data $\mathrm{y} 19 / 0.0 /$

*

vchk $=x($ nelem(imat $)$, imat $)$

iff arg. It. vchk) go to 5

val $=y($ nelem $($ imat $)$,imat $)$

return

c

$5 i=1$

10 if( arg - x(i,imat) ) $100,200,300$

100 if $(i$.eq. 1$)$ go to 200

$r=(\arg -x(i-1$, imat $)) /(x(i, i m a t)-x(i-1$, imat $))$

val $=r^{*}(y(i$, imat $)-y(i-1$, imat $))+y(i-1$, imat $)$

return

$200 \mathrm{val}=\mathrm{y}(\mathrm{i}, \mathrm{imat})$

return

$300 i=i+1$

go to 10

C

end

c

subroutine crossgn ( arg, imat, val)

C --- routine to calculate photoneutron cross-sections for specified material

C

parameter $(\mathrm{nmt}=19)$

C

* $\mathrm{M} 1=\mathrm{Be} 9$

* $\quad M 2=H$ (deuterium)

* $\quad \mathrm{M} 3=\mathrm{U} 235$

* $\quad$ M4 = Pu239

* $\quad \mathrm{M} 5=\mathrm{Li} 6$

* $\quad M 6=\mathrm{U} 238$

* $\quad M 7=\operatorname{Cu} 63(69.2 \%$ abundant $)$

* $\quad \mathrm{M} 8=\mathrm{Cu}-$ natural

* $\quad M 9=016$

* $\mathrm{M} 10=\mathrm{N} 14$

* $\mathrm{M} 11=\operatorname{Ar} 40$

* $\quad M 12=A 127$

* $\quad \mathrm{M} 13=\mathrm{Si} 28$

* M14 = C12

* $\quad$ M15 = FE56

* $\quad \mathrm{M} 16=\mathrm{Cl}$ - natural

* $\quad \mathrm{M17}=\mathrm{P} 31$

* $\mathrm{M} 18=\mathrm{F} 19$ 


$$
\begin{aligned}
& \text { 震 }
\end{aligned}
$$

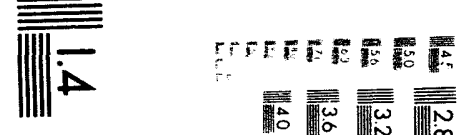

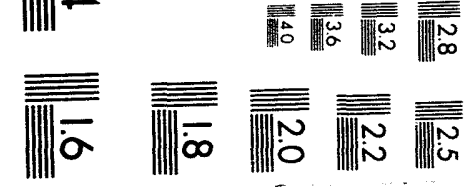




* $\quad$ M19 $=W 74$

dimension nelem(nmt)

c -.- number of elements in cross sectional arrays

data nelem $/ 10,11,14,14,11,14,11,1,10,5,5,4,7$,

$+8,8,10,9,15,14 /$

C

dimension $x(n m t, n m t), y(n m t, n m t)$

equivalence $(x 1(1), x(1,1)),(y 1(1), y(1,1))$

equivalence $(x 2(1), x(1,2)),(y 2(1), y(1,2))$

equivalence $(x 3(1), x(1,3)),(y 3(1), y(1,3))$

equivalence $(x 4(1), x(1,4)),(y 4(1), y(1,4))$

equivalence $(x 5(1), x(1,5)),(y 5(1), y(1,5))$

equivalence $(x 6(1), x(1,6)),(y 6(1), y(1,6))$

equivalence $(x 7(1), x(1,7)),(y 7(1), y(1,7))$

equivalence $(x 8(1), x(1,8)),(y 8(1), y(1,8))$

equivalence $(x 9(1), x(1,9)),(y 9(1), y(1,9))$

equivalence $(x 10(1), x(1,10)),(y 10(1), y(1,10))$

equivalence $(x 11(1), x(1,11)),(y 11(1), y(1,11))$

equivalence $(x 12(1), x(1,12)),(y 12(1), y(1,12))$

equivalence $(x 13(1), x(1,13)),(y 13(1), y(1,13))$

equivalence $(x 14(1), x(1,14)),(y 14(1), y(1,14))$

equivalence $(x 15(1), x(1,15)),(y 15(1), y(1,15))$

equivalence $(x 16(1), x(1,16)),(y 16(1), y(1,16))$

equivalence $(x 17(1), x(1,17)),(y 17(1), y(1,17))$

equivalence $(x 18(1), x(1,18)),(y 18(1), y(1,18))$

equivalence $(x 19(1), x(1,19)),(y 19(1), y(1,19))$

*

dimension $x 1(10), y 1(10), x 2(11), y 2(11), x 3(14), y 3(14)$,

$+x 4(14), y 4(14), x 5(11), y 5(11), x 6(14), y 6(14), x 7(11), y 7(11)$,

$+x 8(1), y 8(1), x 9(10), y 9(10), x 10(5), y 10(5), x 11(5), y 11(5)$,

$+x 12(4), y 12(4), x 13(7), y 13(7), x 14(8), y 14(8)$,

$+\quad x 15(8), y 15(8), x 16(10), y 16(10), x 17(9), y 17(9)$,

$+\quad x 18(15), y 18(15), x 19(14), y 19(14)$

* 1

* Be 9 (MeV vs mB) *** 4/90 - Bulow and Forkman (Seems high!)

* DATA X1/1.7, 1.8, 2.1, 2.6, 3.0, 3.5, 6.0, 10.0,12.0.14.1

* DATA Y1/0.0, 2.0,0.5, 1.3, 5.0, 0.5, 4.0,4.0,4.0,4.0/

* 1

* Be 9 (MeV vs mB) ***12/90 - M.J. Jakobson '61 (Phys. Rev. 123,\#1,229)

* \& L.W. Jones '53, (Phys. Review. 91,\#3,699)

DATA $X 1 / 1.7,1.8,2.2,2.6,3.0,3.2,4.2,4.6,9.0,18.0 /$

DATA Y $1 / 0.0,1.15,0.3,0.5,1.2,0.4,0.2,1.15,0.8,4.0 /$

* 2 
* H 2 (DEUTERIUM) *** 5/90 - Bulow and Forkman

DATA $\times 2 / 2.2,2.231,3.15,4.65,4.99,7.37,9.01,10.9,13.66$,

$+\quad 16.6,20.0 /$

DATA Y $2 / 0 ., 1.3,1.61,2.44,2.45,1.88,1.54,1.25,1.0, .76, .6 /$

* 3

* U 235 SS Dietricj LLNL 1986 (total yield curve). Includes photofission

* photofission energy threshold $=5.8 \mathrm{MeV}$, photoneutron thres $=5.3 \mathrm{MeV}$

DATA $\times 3 / 5.3$, 6., 7., 8., 9., 10., 11., 12., 13., 14.,

$+\quad 15 ., 16 ., 17 ., 18.1$

DATA Y3/0.0, 25., 50.,100., 200., 500., 880., 1000., 1200., 1500.,

$+\quad 1300 ., 900 ., 700 ., 500.1$

* 4

* PU 239 SS Dietrich LLNL 1986 (total yield curve).

* photofission energy thres $=5.6 \mathrm{Mev}$, photoneutron energy thres $=5.6 \mathrm{MeV}$

DATA X4/5.6, 6.0, 7.0, 8.0, 9.0, 10., 11., 12., 13.,

$+\quad 14 ., 15.0,16.0,17.0,18.0 /$

DATA Y4 / 0.0, 20., 60., 125., 250., 600., 1100., 1300., 1400.,

$+\quad$ 1650., 1400., 900., 800., 500. /

$* 5$

* Li 6

DATA $\times 5 / 5.6,5.701,9.49,11.99,15.0,16.2,19.61,21.99,29.49$, $+\quad 30.27,32.211$

DATA y $5 / 0.0, .23,1.36,1.69,1.25,1.47, .89,1.01, .77,1.97, .66 /$

* 6

* U 238 SS Dietrich LLNL 1986 (total yield curve).

* photofission energy thres $=5.8 \mathrm{Mev}$, photoneutron energy thres $=6.1 \mathrm{MeV}$

DATA X6/5.8, 6., 7., 8. , 9. , 10., 11., 12., 13., 14.,

$+\quad 15 ., 16 ., 17 ., 18.1$

DATA Y6/0.0,24.0, 40., 100., 200., 450., 700., 800., 1100., 1200.,

$* 7$

$+\quad 1150 ., 800 ., 550 ., 400.1$

* Cu 63 (69.3\% abundant) (MeV \& mb* *11/90)

data $x 7 / 10.8,12 ., 14 ., 16 ., 18 ., 20 ., 22 ., 23 ., 24 ., 26 ., 28 . /$

* 8

data $y 7 / 0.0,10 ., 30 ., 65 ., 60 ., 40 ., 25 ., 30 ., 15 ., 15 ., 5 . /$

* dummy

data $\times 8 / 0.0 /$

data $y 8 / 0.0 /$

* 9

* O 16 ( yield cross section: MeV \& mb * $2 / 90$ )

DATA $\times 9 / 15.7,17.0,18.0,19.0,20.0,22.0,23.0,24.0,29.0,40.0 /$

DATA Y9/ $0.0,2.0,0.5,2.0,0.5,10.0,7.0,10.0,4.0,1.5 /$

* 10

${ }^{*} N 14$ ( yield cross section: MeV \& mb * $2 / 90$ ) 
DATA $\times 10 / 10.6,17.0,2 \iota .0,22.5,25.0 /$

DATA $Y 10 / 0.0,1.4,5.6,15.3,0.6 /$

* 11

* Ar 40 (yield cross section: MeV \& mb **2/90)

DATA $\times 11 / 9.9,17.5,18.5,22.0,30.0 /$

DATA $Y 11 / 0.0,48.0,44.0,52.0,12.0 /$

* 12

* Al 27 (yield cross section: MeV \& mb * $2 / 90$ )

DATA $\times 12 / 13.1,22.0,26.0,40.0 /$

DATA Y $12 / 0.0,15.0,8.0,3.0 /$

* 13

* Si 28 (yield cross section: MeV \& mb **2/90)

DATA $\times 13 / 17.2,19.0,20.0,21.0,23.0,25.0,35.0 /$

DATA Y13/0.0,14.0,10.0,16.0, 8.0, 5.0, 3.0/

* 14

* C 12 (yield cross section: MeV \& mb **2/90)

data $\times 14 / 18.7,20.5,22.0,23.0,25.0,25.5,26.0,32.5 /$

data y $14 / 0.0,1.3,6.7,7.2,3.3,4.2,3.3,1.3 /$

* 15

* FE 56 (yield cross section: MeV \& mb ** 6/90 -- bulow \& forkman) data $\times 15 / 11.2,11.6,12.4,14.0,17.0,20.3,22.5,24.0 /$

data $y 15 / 0.0,7.0,7.0,20.0,65.0,50.0,25.0,15.0 /$

* 16

* Cl natural (yield cross section: MeV \& mb **11/90)

data $\times 16 / 10.3,12 ., 14 ., 16 ., 18 ., 20 ., 22 ., 24 ., 26 ., 28$. /

data y $16 / 0.0,1.0,4.0,12 ., 23 ., 25 ., 23 ., 20 ., 16 ., 13 . /$

* 17

* P31 (yield cross section: MeV \& mb **11/90)

data $x 17 / 12.3,14 ., 16 ., 18 ., 20 ., 22 ., 24 ., 26 ., 28$. /

data $y 17 / 0.0,2.0,5.0,12 ., 13.5,18 ., 15 ., 12 ., 11 . /$

* 18

* F19 (yield cross section: MeV \& mb * * 11/90)

data $\times 18 / 10.4,11 ., 12 ., 13 ., 14 ., 15 ., 16 ., 17 ., 18 ., 19 .$,

+ 20., 22., 24., 26., 28.1

data $y 18 / 0.0,0.25,4.0,2.0,3.0,2.5,3.5,4.0,5.0,5.0$,

$+6.5,9.0,11 ., 10 ., 12.1$

* 19

* W74 (yield cross section: MeV \& mb * * 11/90)

data $x 19 / 8 ., 9 ., 10 ., 11 ., 12 ., 13 ., 14 ., 15 ., 16 .$,

$+17 ., 18 ., 19 ., 20 ., 21.1$

data y19/0.0, 50., 90., 175., 300., 410., 425., 550., 550.,

*

$+450 ., 325 ., 225 ., 190 ., 150$. / 


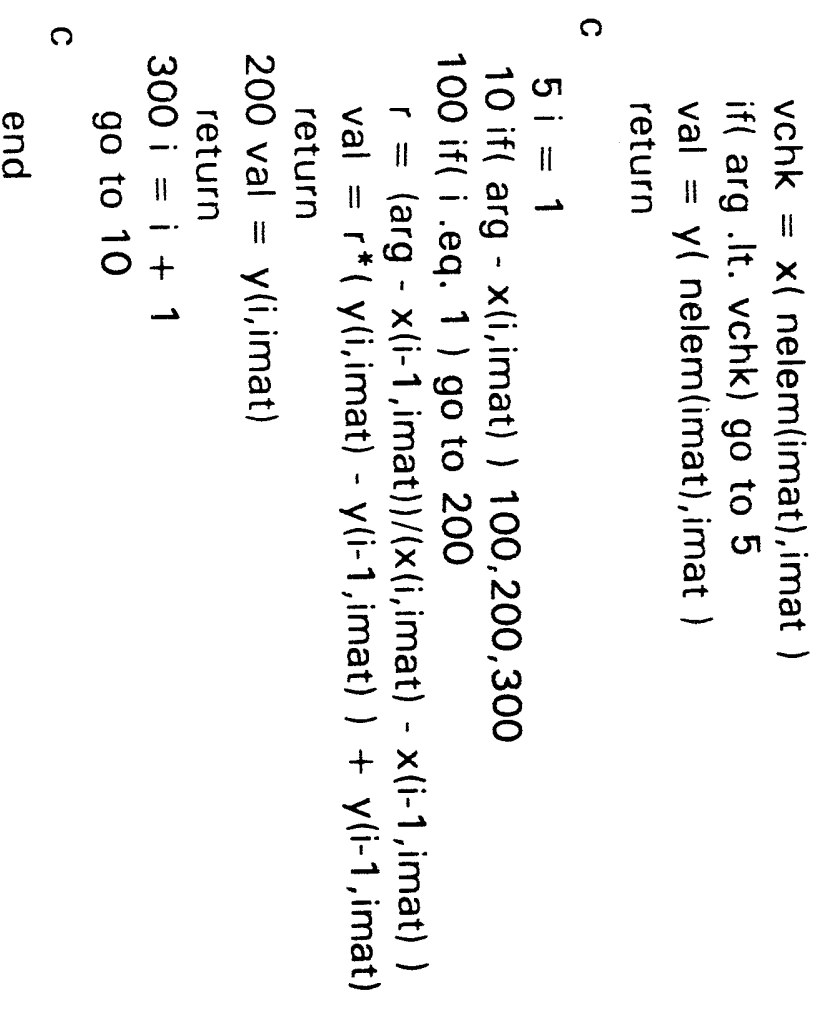



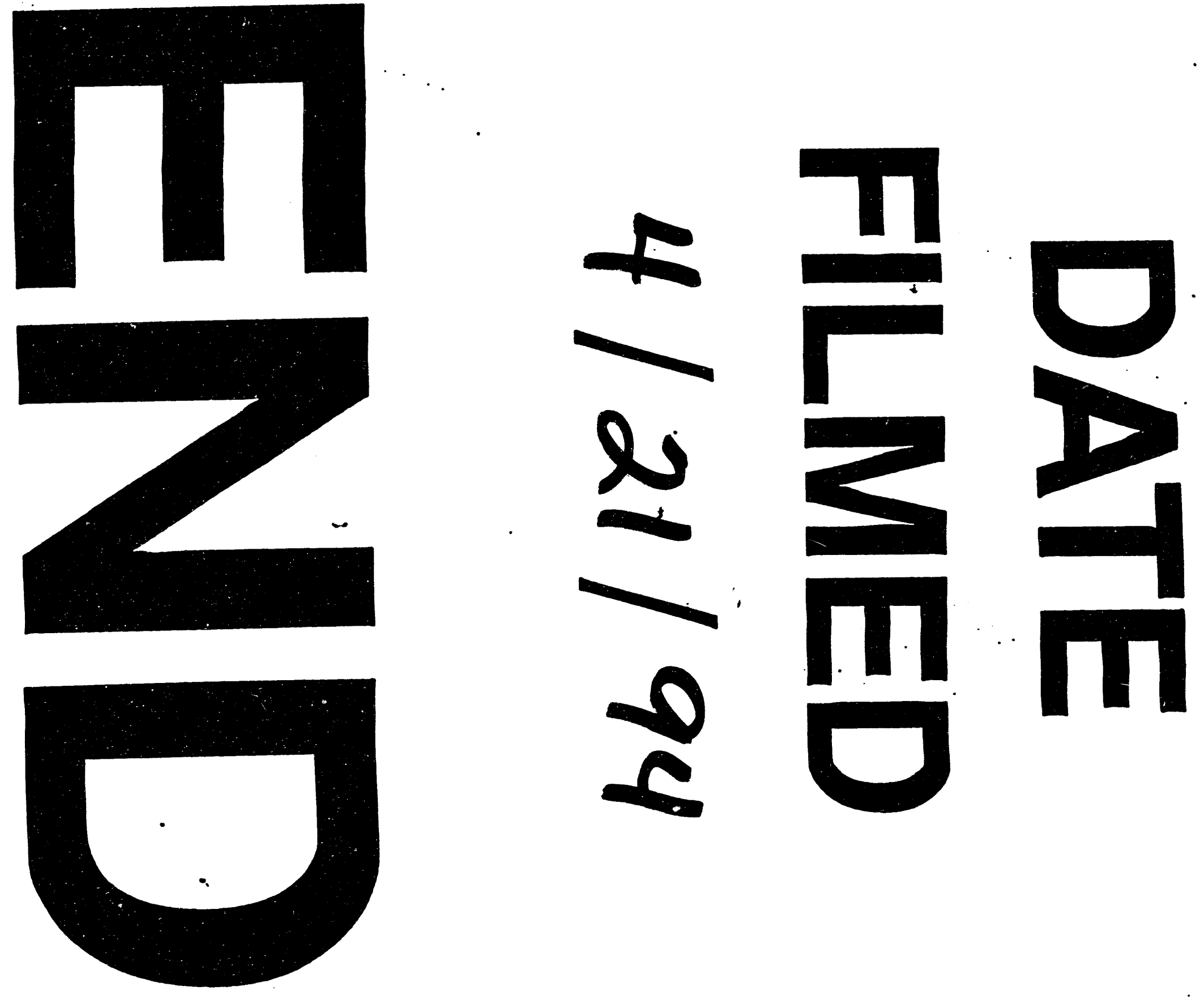
